\title{
Design and optimisation of a \\ laboratory scale microwave furnace for heating titanomagnetite ironsand
}

A thesis submitted in fulfillment of the requirements for the degree of

\author{
Master of Engineering
}

at

Victoria University of Wellington

by

Martin Markwitz

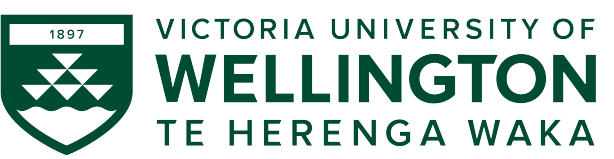





\section{Abstract}

TTM (Titanomagnetite) ironsand is an abundant source of iron oxide on the western beaches of the Waikato and Auckland regions in New Zealand, with chemical formula $\mathrm{Ti}_{\mathrm{x}} \mathrm{Fe}_{3-\mathrm{x}} \mathrm{O}_{4}(\mathrm{x}=0.27)$. This ironsand has been used for the last four decades to produce steel in New Zealand, but the reduction process releases large amounts of carbon dioxide. This is because coal is used as the primary reducing agent. Using hydrogen gas instead as a reducing agent, it is possible to reduce ironsand while avoiding the excessive production of carbon dioxide. In addition, standard electrical heating methods are generally limited by low power transfer rates to the steelmaking reactants. Microwave heating is an alternative heating method, shown to be good candidate for ironsand heating via direct power transfer to the NZ ironsand itself due to its excellent microwave absorbing features. This research focuses on modelling the dynamics of microwave power transfer in a resonant microwave cavity, and refines a computational model to improve the modelling capability of such a process.

Microwave heating is known to demonstrate high direct power transfer rates to microwave absorbing materials, such as TTM ironsand. The microwave heating of ironsand as a candidate heating method is shown in this work by the optimisation of the resonant performance of a custom-built laboratory scale microwave cavity to heat ironsand to over $1000^{\circ} \mathrm{C}$. The microwave furnace developed in this work was specifically designed to mimic the contemporary steelmaking standards (i.e., a continuous throughput furnace). A microwave furnace was designed with a computational model, then built and tested in a laboratory. Measurements tracking various energy fluxes throughout the furnace refined an initial transient microwave heating simulation of the computational model with a simple experimental procedure, which exploits the significant variation of the real permittivity $\left(\varepsilon_{r}^{\prime}\right)$ of the ironsand at $T=430^{\circ} \mathrm{C}$. 
From that experimental procedure, a significant variation in microwave absorption was observed as the ironsand passed through its Curie temperature. This effect was reproduced in an initial transient microwave heating simulation. Experimental results were then used to improve simulation accuracy. This encourages further refinement of the computational model as an avenue for future work in this field.

In summary, this research demonstrates the feasibility of designing a microwave furnace for efficiently heating TTM ironsand. It also exhibits the feasibility of simulating microwave heating of TTM ironsand with a computational model. The results from this thesis show promise for further study on the hydrogen reduction of TTM ironsand within a microwave furnace. The findings therein present results which 'set the scene' for for larger-scale zero- $\mathrm{CO}_{2}$ production of techno-economically essential materials via microwave heating. 


\section{Acknowledgements}

Thanks must go to my supervisors Dr. Chris Bumby and Dr. John Kennedy for their continual support throughout this project. You have both challenged me to learn as much as possible throughout this last year and to always keep a high standard when explaining the concepts explored throughout this work. I appreciate the time (and patience where it was necessary) that you both provided me to discuss the project and answer my questions. The help and guidance was invaluable, and I cannot possibly understate how much I appreciate the opportunity to have been given the chance to work on this fascinating project.

My deepest thanks go to John Futter who was instrumental in the building of the cavity, transmission line and for introducing me about the complexities of microwave systems. Thank you for always suggesting great ideas on how to solve problems (of which there were plenty) and always being available for a 'quick question' which often turned into hours of work for me thereafter to confirm what we spoke about.

I also share my gratefulness to the entire Materials team at the National Isotope Centre of the Institute of Geological and Nuclear Sciences for the work space, and a place to put my furnace. I felt welcome to join you at all times and treasure the opportunity I was given. Special thanks to Diego Del Puerto from Callaghan Innovation for your availability for help at any time, and our engaging office chats. I also acknowledge my close colleagues Ao Zhang, Sigit Prabowo, and Shaira Mendoza from Robinson Research Institute, I hope you find my furnace useful for your further work. A special note for Richard Tang from the University of Auckland, it was heaps of fun working with you.

I was grateful to be financially supported by the Ministry of Business, Innovation and Employment Grant for Masters Scholarship 2020 throughout 
this work.

In addition, I thank my closest friends Alex, Gian, Richard, Luke and a range of others who I was able to wind down with (or be wound up by) throughout the years of playing League of Legends together. A short thank you also goes to the friends I made in college, and still keep in touch with. Thanks Fergus, Joshua and Bradley for your company throughout this work and our previous years in undergraduate Physics. I'd also like to acknowledge Dr. Andrew Ross, Peter Coard, David Stead and Dr. Chris Lepper from the School of Chemical and Physical Sciences for being very welcoming whenever I managed to make it to the Laby building. Thank you also Alice, for your help with reviewing parts of my thesis.

A very personal thank you to Michelle, you kept me grounded throughout this project. I always feel lucky to see you (and cook with you!) after a long day's work. I cherish you always being there for me regardless of how busy and stressed we both were throughout our studies.

I finish by thanking my family; Andreas, Susanne and Richard Markwitz. I appreciate everything you've given for me up until this stage in my life, and treasure everything that has come, and look forward to what will. Ich danke auch Marianne Hertler, Christine Hertler und Karin Markwitz für all die Videoanrufe, ich freue much schon euch alle wieder zu sehen. 


\section{Contents}

1 Introduction $\quad 1$

1.1 Steelmaking . . . . . . . . . . . . . . . . . . . . 1

1.2 New Zealand ironsand processing . . . . . . . . . . . . . . . . 1

$1.3 \mathrm{Aim} \ldots \ldots \ldots . \ldots \ldots$

1.4 Problem statement . . . . . . . . . . . . . . . . . 3

1.5 Thesis structure . . . . . . . . . . . . . . . 3

2 Background $\quad 7$

2.1 Introduction to electromagnetism . . . . . . . . . . . . . 7

2.2 Conducting cavities . . . . . . . . . . . . . . . . . . 10

2.2.1 Rectangular RF waveguides . . . . . . . . . . . . 10

2.2.2 Cylindrical RF systems . . . . . . . . . . . . . . . . . . 14

2.2.3 Resonating cavity design procedure . . . . . . . . . . 16

2.3 Thermal losses . . . . . . . . . . . . . . . . . . . . . . . . . . 18

2.4 Using COMSOL Multiphysics . . . . . . . . . . . . . . . 20

2.4.1 Introduction to FEM simulations . . . . . . . . . . . 21

2.4.2 Equations of COMSOL Multiphysics . . . . . . . . 22

2.4.3 Simple resonating cavity . . . . . . . . . . . . 25

2.5 Model material properties . . . . . . . . . . . . . . . 26

2.5.1 Boron Nitride . . . . . . . . . . . . . . . . 27

2.5.2 Insulation fibreboard . . . . . . . . . . . . 27

2.5.3 Titanomagnetite ironsand . . . . . . . . . . . . . . 29

3 Initial prototype furnace modelling 33

3.1 Cavity geometry and design criteria . . . . . . . . . . . . 33

3.2 Input waveguide orientation . . . . . . . . . . . . . . 35

3.3 Beyond-cutoff waveguides . . . . . . . . . . . . . 36 
3.4 Determining basic cavity dimensions . . . . . . . . . . . . . 38

3.5 Thermal insulation . . . . . . . . . . . . . . . . . . . 42

3.6 Optical access . . . . . . . . . . . . . . . . 44

4 Experimental methods $\quad 49$

4.1 Transmission line components . . . . . . . . . . . . . . . . . . . 49

4.1.1 Magnetron launch . . . . . . . . . . . . . . . . . 50

4.1.2 Circulator . . . . . . . . . . . . . . . . . . 52

4.1 .3 Terminator .................. 53

4.1.4 Three-stub tuner . . . . . . . . . . . . . . 53

4.1.5 Dual-directional coupler . . . . . . . . . . . . 55

4.1.6 Microwave cavity . . . . . . . . . . . . . 56

4.2 Experimental setup . . . . . . . . . . . . . . . 57

4.3 Measurement processes . . . . . . . . . . . . . . . 60

4.3.1 Microwave leakage detection . . . . . . . . . . 60

4.3.2 AD8317 RF-DC detectors . . . . . . . . . . . . 61

4.3.3 Water calorimetry. . . . . . . . . . . 65

4.3.4 Pyrometers . . . . . . . . . . . . . . . 68

4.4 Explorational experimental findings . . . . . . . . . . . . 71

4.4.1 Plasma production . . . . . . . . . . . . . . 71

4.4.2 Lichtenberg figure production . . . . . . . . . . . 73

4.4.3 Partial ironsand sintering . . . . . . . . . . . 74

4.4.4 XRD analysis . . . . . . . . . . . . . 76

4.5 Experimental procedure . . . . . . . . . . . . . 78

$\begin{array}{llr}5 & \text { Results } & 81\end{array}$

5.1 Experimental results . . . . . . . . . . . . . . . 81

5.2 Microwave furnace model investigation . . . . . . . . . . . 85

5.3 Modelling results . . . . . . . . . . . . . . . . . . . 86

5.3 .1 Initial model results . . . . . . . . . . . . . . . 87

5.3 .2 Model refinements . . . . . . . . . . . . . . . 90

5.4 Modelling the three-stub tuner . . . . . . . . . . . . . 96

5.4.1 Low temperature stub optimisation . . . . . . . . 97

5.4.2 High temperature stub optimisation. . . . . . . . . . . . 99

5.4.3 Transient models due to stub optimisation . . . . . . . . 103

5.5 Ironsand temperature profile . . . . . . . . . . . . 105 
6 Conclusions and recommendations 109

6.1 Conclusions . . . . . . . . . . . . . . . . . . . . 109

6.2 Recommendations for future work . . . . . . . . . . . . . . 110

6.3 Implications of this thesis . . . . . . . . . . . . . . 111

Bibliography 112

$\begin{array}{ll}\text { List of Figures } & 123\end{array}$

List of Tables 133

$\begin{array}{ll}\text { A Nomenclature } & 135\end{array}$

$\begin{array}{ll}\text { B Supplementary derivations } & 137\end{array}$

B.1 The wave equation . . . . . . . . . . . . . . . . . . 137

B.2 Rectangular waveguide wave equation . . . . . . . . . . . . 138

B.3 Rectangular waveguide cutoff frequencies . . . . . . . . . . . . . 140

B.4 Cylindrical waveguide wave equation . . . . . . . . . . . . . . 142

B.5 Cylindrical resonance and cutoff frequencies . . . . . . . . . . . 144

B.6 Cylindrical beyond-cutoff waveguide . . . . . . . . . . . . . . . . 146

$\begin{array}{lr}\text { C Mathematical identities } & 149\end{array}$

C.1 Vector calculus: Cartesian Coordinates . . . . . . . . . . . . . . 149

C.2 Vector calculus: Cylindrical Coordinates . . . . . . . . . . . . 150

C.3 Fundamental theorems of vector calculus . . . . . . . . . . . . 150

C.4 Vector identities . . . . . . . . . . . . . . . . . . . . 151

D Relevant software interfaces 152

D.1 National Instruments LabVIEW . . . . . . . . . . . . . . . . . . 152

D.2 COMSOL Multiphysics . . . . . . . . . . . . . . 153

D.3 MathWorks MATLAB . . . . . . . . . . . . . . . . 154 


\section{Chapter 1}

\section{Introduction}

\subsection{Steelmaking}

Steelmaking has been essential in the development of modern humanity. The first steps towards modern blast furnace methods were made in the $17^{\text {th }}$ century [1], and methods to improve the process are still being made today. Steel is used everywhere from strengthening buildings, to making cars, to everyday reusable cutlery. The strength of steel is relied on in everyday life and as people develop larger machines and buildings, steel alloys will play an even larger role in the safe long-term usage of these designs. Steelmaking has seen a $140 \%$ increase in production in the last 20 years from 753 million tonnes produced in 1995 to 1808 million tonnes produced in 2018 [2]. Each tonne of reduced iron ore produces approximately 1.8 tonnes [3] of $\mathrm{CO}_{2}$ emissions. In 2014, the production of steel made up $7 \%$ of $\mathrm{CO}_{2}$ of the total emissions from the global use of fossil fuels [4]. This has promoted further interest into the reduction of greenhouse gas emissions throughout the steelmaking process [5-10]. In addition, in 2016, contemporary steelmaking methods have such poor efficiency to the point that worldwide steelmaking processes account for $20 \%$ of industrial power usage [3].

\subsection{New Zealand ironsand processing}

Recent developments in New Zealand have focused on using hydrogen reduction methods as an alternative to the current carbon based methods for steelmaking. 
Much research has been done on the dynamics of the carbon-based reduction process for steelmaking [11-13]. New Zealand has large and easily accessible natural stores of TTM ironsand on the west coast of the North Island which can be extracted and magnetically purified at low cost for the raw ironsand. The TTM ironsand has the stoichiometric form $\mathrm{Fe}_{3-\mathrm{x}} \mathrm{Ti}_{\mathrm{x}} \mathrm{O}_{4}$ with titanium proportion of $\mathrm{x}=0.27$ [14]. The TTM ironsand has the same inverse spinel crystal structure as standard magnetite [15-18]. Due to its titanium concentration, it is not possible to use a conventional blast furnace reduction process, as the titaniumrich slag produced in the blast furnace method will block the furnace [19]. TTM is, therefore, commercially reduced by $\mathrm{CO}_{2}$-intensive direct reduction methods, specifically by use of coal [20]. A new method of TTM ironsand reduction is highly desirable as an economically and ecologically viable method of producing steel. When hydrogen is mixed with pre-processed TTM ironsand in a fluidised bed reactor it has been shown that the ironsand is rapidly reduced at high temperatures $(\mathrm{T}>1000 \mathrm{~K})$ [21]. It has also been shown that New Zealand ironsand does not stick to itself in those conditions [22]. This is due to the production of a titanium oxide rich shell at the surface of each grain of the ironsand which prevents iron-iron contact between grains that would otherwise result in sticking between the particles [23]. This makes New Zealand ironsand the ideal material for a hydrogen-based reduction process.

This approach requires ironsand to be processed in a hydrogen-rich atmosphere at high temperatures, and to achieve those high temperatures an emission-safe heating method must be employed. Electrical heating methods generally power a heating element which in turn heats the desirable object or material via conductive or radiative energy transfer. Magnetite powder has been shown to be an excellent microwave absorbing material [24]. Since TTM is almost structurally identical to magnetite, it is practical to use microwave heating to directly transfer microwave power to the ironsand. Microwave radiation is considered to be electromagnetic radiation with frequencies between $1 \mathrm{GHz}$ and $100 \mathrm{GHz}$ [25]. The frequency of interest in this work is $2.45 \mathrm{GHz}$, a standard in laboratory and commercial microwave applications [26]. The field of engineering associated with this frequency range is known as RF (Radio Frequency) engineering. This requires the development of a microwave furnace which is the core topic of research for this work. The clearest advantage of using a microwave furnace is a greatly increased heat transfer rate over the 
conventional infrared heating methods [27]. The microwave furnace is designed in this work with continuous processing in mind to maximise the ironsand throughput rate. The industrial approach for steelmaking [28-31] is emulated with the design of the open-ended cavity furnace.

\subsection{Aim}

There are two key aims of this project;

1. Design, build and test an optimised laboratory scale microwave furnace for heating NZ ironsand.

2. Develop and refine a finite element model which simulates the heating performance of NZ ironsand in a microwave furnace.

\subsection{Problem statement}

To resolve the aims of this work, a number of questions must be answered.

- What types of resonant modes in a cylindrical conducting geometry are useful for microwave heating a centred rod of TTM ironsand?

- Can the dynamics of the microwave furnace be modelled using finite element modelling software?

- What are the key parameters which affect the heating performance of the furnace that must be simulated in a working finite element model?

These questions outline the general structure of the work done throughout this thesis.

\subsection{Thesis structure}

The relevant theoretical background material and techniques used in this research are introduced in Chapter 2. Chapter 3 details the initial development of the prototype furnace. Then, Chapter 4 discusses experimental components, calibration procedures and measurement methods. Chapter 5 presents experimental and modelling results. Finally, Chapter 6 summarises the work done 
in this research, then indicates avenues for further research with microwave heating of TTM ironsand.

\section{Chapter 2 - Background}

In this chapter, the relevant physical theories and modelling fundamentals are reviewed. Firstly, the physics of propagating and resonating electromagnetic waves within confined conducting geometries is discussed. The fundamental types of thermal losses are introduced and reviewed in the context of a microwave furnace. Multiphysics modelling software is then introduced to simultaneously solve both electromagnetic and thermal properties of the ironsand in the furnace. Finally, this chapter shows the relevant thermal and microwave properties of materials used throughout this research.

\section{Chapter 3 - Initial prototype furnace modelling}

This chapter establishes the initial prototype furnace model used in this work. The various dimensions of the furnace; such as the cavity length, diameter and input waveguide orientation are established. In addition, the effect of adding optical access tubing, large beyond-cutoff waveguides and thermal insulation to the cavity are analysed one-by-one. The finite element model is then optimised for centralised single-mode resonance of a cylindrical resonant cavity. The simulation work from this chapter results in an initial prototype for an experimentally refinable microwave furnace model.

\section{Chapter 4 - Experimental methods}

Chapter 4 discusses RF transmission line components and pieces of equipment used in this work. The experimental setup process is then shown. Furthermore, equipment calibration and error analysis methods are reviewed. Throughout this research, explorational experiments resulted in some findings of interest, and these are also reviewed in this chapter. An experimental method is presented, from which the results are used as the reference for computational model refinement. 


\section{Chapter 5 - Results}

The second to last chapter in this work is the collection of experimental and simulated results. The model's initial results are compared to experimental observation. The investigation then follows where model inaccuracies are corrected by variations of key parameters, to best match experimental results. The optimised combination of stub positions are found and the resulting transient microwave heating simulations are analysed.

\section{Chapter 6 - Conclusions and recommendations}

To conclude the thesis, the work completed throughout the project is summarised. Closing remarks review the various measurement and investigation methods developed throughout this work. This chapter also determines further work to be done on the microwave furnace for commercial processing viability, and hydrogen gas inclusion. 


\section{Chapter 2}

\section{Background}

This chapter begins with an introduction to the electromagnetic theory of transmission lines and resonant cavities. Various types of thermal losses are then reviewed. The FEM (Finite Element Modelling) software is then introduced. This software couples the electromagnetic and thermal properties of materials in a computational model, allowing the electromagnetic and heating properties of the furnace to be explored and optimised. Finally, the materials used in the experiment are shown, establishing a starting point for the material parameters used in simulations shown in later chapters.

\subsection{Introduction to electromagnetism}

The starting point for understanding the electrodynamics of the microwave cavity is the set of Maxwell-Heaviside equations of electromagnetism $[32,33]$. In the context of this thesis, analytical solutions to the Maxwell-Heaviside equations are generally only available for simple geometries, such as rectangular, cylindrical or spherical systems. Those simple geometries in microwave systems often result in restrictions on the propagation wavevectors of the energy in the fields, when confined in conductive geometries $[34,35]$. The fields are considered time-harmonic fields, and are therefore written as $\vec{F}(\vec{r}, t)=\vec{F}_{0}(\vec{r}) \exp (-i \omega t)^{1}$ with angular frequency $\omega$, imaginary number $i=\sqrt{-1}$ and time $t$. The units associated with the various fields and functions of interest are included in Appendix A. The Maxwell-Heaviside equations of electromagnetism in

\footnotetext{
${ }^{1}$ Note that $\vec{F}_{0}$ is a complex-valued vector field.
} 
differential form are

$$
\begin{aligned}
\nabla \cdot \vec{D} & =\rho_{f} \\
\nabla \cdot \vec{B} & =0 \\
\nabla \times \vec{E} & =-\frac{\partial}{\partial t} \vec{B} \\
\nabla \times \vec{H} & =\frac{\partial}{\partial t} \vec{D}+\vec{J}_{f}
\end{aligned}
$$

with $\vec{D}$ the displacement vector field defined as $\vec{D}=\varepsilon_{0} \vec{E}+\vec{P}$, where $\vec{E}$ the electric field and $\vec{P}$ the polarisation field. The permittivity of free space $\varepsilon_{0}$ couples the free charge density $\rho_{f}$ to the the electric field. The auxiliary magnetic vector field $\vec{H}$ is written as $\vec{H}=\vec{B} / \mu_{0}+\vec{M}$, where $\vec{M}$ the magnetisation field and $\vec{B}$ the magnetic field. The permeability of free space $\mu_{0}$ couples the electric free current density $\vec{J}_{f}$ to the magnetic field. The current density and charge density are related at each point in space by the continuity equation [36]

$$
\nabla \cdot \vec{J}_{f}+\frac{\partial}{\partial t} \rho_{f}=0
$$

If the response of a material experiencing an electromagnetic field is linear, time invariant, and isotropic [36], the displacement field $\vec{D}$ and magnetic field $\vec{B}$ can written as $[32]$

$$
\begin{aligned}
\vec{D} & =\varepsilon \vec{E} \\
\vec{B} & =\mu \vec{H}
\end{aligned}
$$

where $\varepsilon$ and $\mu$ are material-specific properties. Generally, the materialspecific properties are given with respect to values in a vacuum; $\varepsilon=\varepsilon_{0} \varepsilon_{r}$ and $\mu=\mu_{0} \mu_{r}$. Due to the constituents of materials such as the free charge carriers or crystal lattice site occupants, materials react to excitations produced by incident electromagnetic fields. These responses range from resonance in lattice site occupants with the incoming fields to the transfer of energy stored in the electromagnetic field to the free charge carriers within the material. The relative permittivity $\varepsilon_{r}$ and relative permeability $\mu_{r}$ of a material describe this complex system of responses to electromagnetic excitations ${ }^{2}$. A material's

\footnotetext{
${ }^{2}$ Materials in which $\varepsilon_{r}>1$ and $\mu_{r}=1$ are considered dielectrics [36]
} 
electromagnetic response may have some magnitude and phase delay which vary from the input field's characteristics, so it is necessary that the relative permittivity and relative permeability are complex-valued;

$$
\begin{aligned}
& \varepsilon_{r}=\varepsilon_{r}^{\prime}-i \varepsilon_{r}^{\prime \prime} \\
& \mu_{r}=\mu_{r}^{\prime}-i \mu_{r}^{\prime \prime}
\end{aligned}
$$

In the case of the relative permittivity $\varepsilon_{r}$, the imaginary component corresponds to dielectric losses as work is done by an incoming field to displace the material constituents from their equilibrium positions. The same type of loss is analogously true for the description of the imaginary permeability. The real components of the permittivity and permeability describe how much electromagnetic field energy can be stored in the material. The energy density $U_{E M}$ of an electromagnetic field is given by [36]

$$
U_{E M}=\frac{1}{2}(\vec{E} \cdot \vec{D}+\vec{B} \cdot \vec{H})
$$

A harmonic electromagnetic field exciting a material may also generate currents of charge carriers ${ }^{3}$ within that material via the following definition of the free current density in (Eq. 2.1.6), defined below:

$$
\overrightarrow{J_{f}}=\sigma \vec{E}
$$

where $\sigma$ is the electrical conductivity. This definition relates the electric field to the induced current density within a material, resulting in an analogous vector form of Ohm's law [36]. The displacement of these charge carriers requires work to be done on them, defined as conductive loss. If the current density term $\vec{J}_{f}$ and displacement field expression $\vec{D}$ are placed into (Eq. 2.1.1d), the definition of the total permittivity is rewritten (with inclusion of conductive losses) as

$$
\varepsilon_{r}=\varepsilon_{r}^{\prime}-i\left(\varepsilon_{r}^{\prime \prime}+\frac{\sigma}{\varepsilon_{0} \omega}\right)
$$

It is also important to note that these material specific parameters $\left(\varepsilon_{r}, \mu_{r}, \sigma\right)$ are generally frequency, and temperature dependent [24]. In this work, only one frequency is used, $(f=2.45 \mathrm{GHz})$ so the bulk conductive and dielectric losses

\footnotetext{
${ }^{3}$ The charge carriers may either be electrons or the lack of electrons, known as a 'hole' [37].
} 
are indistinguishable from one another. In addition, there are no equivalent conductive magnetic losses, as no magnetic charges or currents appear to exist.

\subsection{Conducting cavities}

This section summarises the essential mathematics of electromagnetic field confining conducting geometries. The differential equations governing propagation of electromagnetic radiation within rectangular and cylindrical cavities are shown. The fundamental modes of propagation of electromagnetic radiation through such geometries are hence obtained, along with their corresponding cutoff frequencies. This works for all frequencies in the electromagnetic spectrum, however, only the $2.45 \mathrm{GHz}$ frequency is considered for this work.

\subsubsection{Rectangular RF waveguides}

An essential part of this research project is the ability to control high-frequency electromagnetic waves, specifically their propagation and resonance. Since the microwave source is not at the same location as the microwave load, and sending microwave power through free space is highly energy inefficient ${ }^{4}$, the natural choice is to use a waveguide to direct the microwave propagation [38]. An ideal rectangular RF waveguide is an infinitely long cavity with width $a$ and height $b=a / 2$ with the dimensions chosen to result in single-mode transfer of RF power. Here, the specific waveguide geometry of interest is a WR340 (Waveguide Rectangular), which takes its name from the $86.36 \mathrm{~mm}$ width which corresponds to a width of 3.4 inches and results in propagation of the $\mathrm{TE}_{10}$ mode of $2.45 \mathrm{GHz}$ microwave power. The WR340 waveguide dimensions are international standard waveguide dimensions [39], and is commonly used in commercial household microwave ovens. No other electromagnetic modes at that frequency are propagated along the waveguide due to being 'beyond-cutoff' for those modes. The BC (Beyond-Cutoff) concept is discussed following the establishment of the cutoff frequency of the WR340 waveguide.

The interior of the rectangular cavity is considered a vacuum; $\rho_{f}=0$, $\overrightarrow{J_{f}}=\overrightarrow{0}$ with $\varepsilon=\varepsilon_{0}$ and $\mu=\mu_{0}$. The body of the cavity (the copper-coloured section in Figure 2.1) is considered a perfectly conducting material $\sigma \rightarrow \infty$. For a $2.45 \mathrm{GHz}$ propagating microwave, this is a good approximation if the

\footnotetext{
${ }^{4}$ In free space there is a $\frac{1}{d^{2}}$ loss in field intensity.
} 


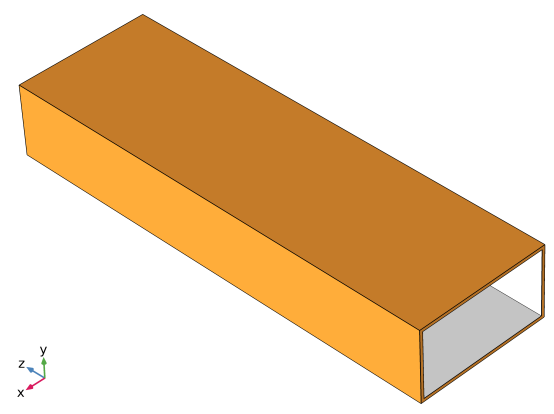

Figure 2.1: A model of a rectangular WR340 waveguide with width $86.36 \mathrm{~mm}$ and height $43.18 \mathrm{~mm}$ built in COMSOL Multiphysics. In this work, $2.45 \mathrm{GHz}$ radiation is launched into the bottom-right of the model. This microwave power is propagated through the waveguide in the characteristic $\mathrm{TE}_{10}$ mode profile, and out the other side of the waveguide (which cannot be seen in this image).

term associated with the current density $\vec{J}_{f}$ in the modified Ampère's law (Eq. 2.1.1d) is orders of magnitude greater than the term associated with the displacement field $\vec{D}$. A short calculation results in a displacement current contribution of $\varepsilon_{0} \omega=1.36 \times 10^{-1} \mathrm{~S} / \mathrm{m}$. The electrical conductivity of copper is $\sigma=5.87 \times 10^{7} \mathrm{~S} / \mathrm{m}[40]\left(\right.$ at $T=25^{\circ} \mathrm{C}$ ), so the assumption for it being a perfect conductor is suitable. Microwave power will propagate within this conducting cavity geometry due to the large conductivity of copper. Two conditions for the coupled electromagnetic fields at the boundary between the 'perfectly' conducting copper and the waveguide vacuum are established. Firstly, the scalar potential $V$ must be constant within the conductor, due to electric charge within the conductor freely being able to distribute itself to oppose any applied electric field. Since $\vec{E}=-\nabla V$, this implies that the electric field must be perpendicular to the interface ${ }^{5}$. Given that the electric field is zero in a perfect conductor (Eq. 2.1.1c) states that the magnetic field is unchanging. Since magnetic fields always form closed loops from (Eq. 2.1.1b), no magnetic field lines can cross that interface. Thus the magnetic field perpendicular to the interface must therefore be zero. Written compactly, the boundary conditions for an electromagnetic field at the interface between vacuum and a perfect

\footnotetext{
${ }^{5}$ The perpendicular direction to the interface is the only 'direction' in which the scalar potential can vary between a vacuum and perfect conductor.
} 
conductor are

$$
\begin{aligned}
E_{\|} & =0 \\
H_{\perp} & =0
\end{aligned}
$$

The Poynting vector, defined as $\vec{S}=\vec{E} \times \vec{H}$ [41] is also parallel to the interface. This is due to the parallel nature of the magnetic field, and the perpendicular nature of the electric field at the interface. This means that the electromagnetic power is propagated within the boundaries of the conducting waveguide. The calculation of Maxwell's equations (Eqs. 2.1.1a, 2.1.1b, 2.1.1c, 2.1.1d) in a confined conducting cavity lead to simultaneous differential equations for the longitudinal electric and magnetic fields:

$$
\begin{aligned}
& {\left[\frac{\partial^{2}}{\partial x^{2}}+\frac{\partial^{2}}{\partial y^{2}}+\left(\frac{\omega}{c}\right)^{2}-k^{2}\right] E_{z}=0} \\
& {\left[\frac{\partial^{2}}{\partial x^{2}}+\frac{\partial^{2}}{\partial y^{2}}+\left(\frac{\omega}{c}\right)^{2}-k^{2}\right] H_{z}=0}
\end{aligned}
$$

Details on this calculation starting with the Maxwell-Heaviside equations in free space, and using the boundary conditions established in (Eqs. 2.2.1a, 2.2.1b) are included in Appendix B. Either $E_{z}$ or $H_{z}$ can be chosen to be zero ${ }^{6}$. If the electric field along the propagation direction is zero (i.e. $E_{z}=0$ ), it results in TE (Transverse Electric) wave propagation. In the case that the magnetic field is zero along the propagation direction (i.e. $H_{z}=0$ ), TM (Transverse Magnetic) wave propagation is observed. Starting with the TE mode $E_{z}=0$, it can be shown that $H_{z}$ is uniquely determined. Then, analytic solutions for all components of both the electric and magnetic fields are calculable via the Maxwell-Heaviside equations. This process begins with separation of variables applied on $H_{z}$ or $E_{z}$, and results in an expression relating the geometry of the cavity $(a, b)$ with propagating frequencies $f_{m n}$. Both $m$ and $n$ are non-negative integers, which determines uniquely the specific mode profile. In the case of a TM mode both $m, n \geq 1$, otherwise the field is trivialised, as the $\mathrm{TM}_{\mathrm{mn}}$ mode has a $\sin (m \pi x / a) \sin (n \pi y / b)$ profile for $E_{z}$. The specific mode cutoff

\footnotetext{
${ }^{6}$ If both are zero, then it is a TEM (Transverse Electromagnetic) mode. These types of modes do not propagate within rectangular systems as the resulting fields are trivialised.
} 
frequencies are given by

$$
f_{m n}=\frac{c}{2} \sqrt{\left(\frac{m}{a}\right)^{2}+\left(\frac{n}{b}\right)^{2}}
$$

Details on this calculation are included in the Appendix B. The mode which is determined by unique combinations of $m$ and $n$ in which the electromagnetic field propagates with is associated with a 'cutoff' frequency given by (Eq. 2.2.3). Electromagnetic waves of that mode below the specific frequency cannot propagate within that waveguide, as the wavenumber $k$ becomes purely imaginary. This results in an evanescent wave which does not transport electromagnetic energy ${ }^{7}$. If both $m, n=0$ then the frequency associated with that mode is zero (a trivial solution), so there is a simple restriction that at least one of $m, n$ is nonzero in the TE case. This particular combination of WR340 dimensions is used to allow for mono-mode transport of the $\mathrm{TE}_{10}$ mode of $2.45 \mathrm{GHz}$ microwave power. A few specific cutoff frequencies $f_{m n}$ for the TE modes are written in Table 2.1. Except in the $m=1, n=0$, and $m=0, n=1$ modes cases, the cutoff frequencies are identical between TE and TM modes.

\begin{tabular}{||c|c||}
\hline TE-mode & $f_{m n}[\mathrm{GHz}]$ \\
\hline \hline 10 & 1.7357 \\
\hline 01 & 3.4714 \\
\hline 11 & 3.8812 \\
\hline 20 & 3.4714 \\
\hline 02 & 6.9429 \\
\hline 21 & 4.9093 \\
\hline 12 & 7.1565 \\
\hline 22 & 7.7623 \\
\hline
\end{tabular}

Table 2.1: The lowest cutoff frequencies for the $\mathrm{TE}_{\mathrm{mn}}$ modes of the rectangular waveguide. These frequencies are found by solving (Eq. 2.2.3) for $f_{m n}$ with $a=86.36 \mathrm{~mm}$ and $b=43.18 \mathrm{~mm}$.

If a $2.45 \mathrm{GHz}$ field is input in an empty WR340 waveguide, only the $\mathrm{TE}_{10}$ mode can propagate. A consequence of this is that the $\mathrm{TE}_{10}$ mode carries all of the microwave power associated with the input field along the input waveguide.

\footnotetext{
${ }^{7}$ Details and a proof of this are again in Appendix B.
} 
Every higher order mode is beyond its associated cutoff frequency (clearly seen in Table 2.1) for the dimensions of the WR340 waveguide.

\subsubsection{Cylindrical RF systems}

Cylindrical resonant RF systems are essential to understanding of the microwave furnace model studied in this work. This section deals with the essential mathematics in understanding electromagnetic resonances in a cylindrical geometry. This concludes with an evaluation of resonant modes in a cylindrical microwave cavity. A resonant cylindrical cavity has specific restrictions on cavity dimensions, which result in unique electromagnetic mode profiles and characteristics.

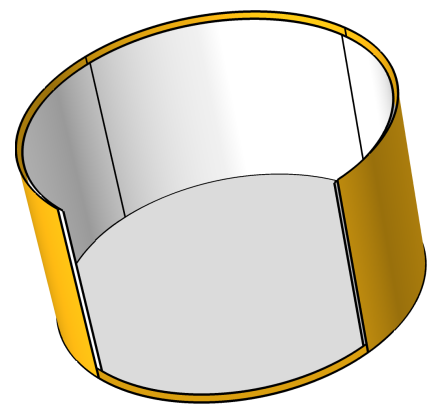

Figure 2.2: A cut-open view of a closed 3D resonant cavity model with radius $R$ and length $L$ built in COMSOL Multiphysics. For any specific frequency, various combinations of radii and length result in resonant modes within the cavity.

The coordinate system is cylindrical with cavity length $L$ along direction $z$, radius $R$ along radial direction $r$ and azimuthal angle $\varphi$. The electromagnetic field is again periodic in time, with non-negative rotational number $l \geq 0$. The rotational number $l$ determines the number of cycles that the electromagnetic field undergoes around the azimuth of the cylindrical cavity ${ }^{8}$. The result of calculation of the longitudinal fields starting with the Maxwell-Heaviside

\footnotetext{
${ }^{8}$ The case that $l=0$ results with complete rotational field symmetry around the central axis.
} 
equations, is differential equations that govern $E_{z}$ and $H_{z}$ :

$$
\begin{aligned}
& {\left[r^{2} \frac{\partial^{2}}{\partial r^{2}}+r \frac{\partial}{\partial r}+r^{2}\left(\frac{\omega^{2}}{c^{2}}-k^{2}\right)-l^{2}\right] E_{z}=0} \\
& {\left[r^{2} \frac{\partial^{2}}{\partial r^{2}}+r \frac{\partial}{\partial r}+r^{2}\left(\frac{\omega^{2}}{c^{2}}-k^{2}\right)-l^{2}\right] H_{z}=0}
\end{aligned}
$$

where again, the details on the calculation are in Appendix B. The calculation of resonant modes of an empty cylindrical cavity will prove to be useful for performance verification of the finite-element modelling software. A closedended resonant cavity (i.e. Figure 2.2) imposes the propagation wavenumber $k$ in (Eqs. 2.2.4a, 2.2.4b) to a fixed real positive number $k_{z}$. Details on resonant frequencies of cylindrical closed-ended cavities are well known [38], and are reviewed here. The type of function that solves the types of differential equations from (Eqs. 2.2.4a, 2.2.4b) is known as a Bessel function [42]. The calculation results in cutoff frequencies again associated with waveguide/cavity dimensions.

For each cavity dimension $R, L$ and parameters $l, m, n$, a unique TM resonant frequency $f \equiv f_{l m n, \mathrm{TM}}$ and $f \equiv f_{l m n, \mathrm{TE}}$ can be found (the calculation is detailed in Appendix B). The resonant frequency of a specific combination of cavity dimensions is determined via the radial $\left(\Gamma_{l m}\right)$ and longitudinal $\left(k_{z}\right)$ wavenumbers. These wavenumbers are restricted to certain values due to the boundary conditions (Eqs. 2.2.1a, 2.2.1b) of the perfect conductor around the cylindrical cavity. The longitudinal wavenumber $k_{z}$ is restricted to $k_{z}=n \pi / L$ with $n>0$ in the case of a TE mode ${ }^{9}$, and $n \geq 0$ in the case of a TM mode. The radial wavenumber $\Gamma_{l m}$ is restricted by the zeros $\chi_{l m}$ of the Bessel function $\Gamma_{l m} \equiv \chi_{l m} / R$ while resonating in a TM mode. The radial wavenumber $\Gamma_{l m}$ can also be restricted by the zeros of the derivatives of the Bessel function $\chi_{l m}^{\prime}$, given as $\Gamma_{l m} \equiv \chi_{l m}^{\prime} / R$ while resonating in a TE mode. Some zeros of the Bessel function, and the derivative thereof associated with each resonant mode type are presented in Table 2.2 .

The resonant frequencies associated with the TM and TE modes of a

\footnotetext{
${ }^{9}$ This is due to the longitudinal field $E_{z}$ having a $\sin (n \pi z / L)$ profile.
} 


\begin{tabular}{||c|c||c|c||}
\hline TE-mode & $\chi_{l m}^{\prime}$ & TM-mode & $\chi_{l m}$ \\
\hline \hline 01 & 3.8317 & 01 & 2.4048 \\
\hline 02 & 7.0156 & 02 & 5.5201 \\
\hline $\mathbf{1 1}$ & $\mathbf{1 . 8 4 1 2}$ & 11 & 3.8317 \\
\hline 12 & 5.3314 & 12 & 7.0156 \\
\hline 21 & 3.0542 & 21 & 5.1356 \\
\hline 22 & 6.7061 & 22 & 8.4172 \\
\hline
\end{tabular}

Table 2.2: The first two zeros of $J_{l}(\Gamma r)$ and $J_{l}^{\prime}(\Gamma r)$ are tabulated with $l=0,1,2$. Note especially the low $\mathrm{TE}_{11}$ mode Bessel function solution.

cylindrical cavity are thus calculated via

$$
\begin{aligned}
f_{l m n, \mathrm{TM}} & =\frac{c}{2} \sqrt{\left(\frac{\chi_{l m}}{\pi R}\right)^{2}+\left(\frac{n}{L}\right)^{2}} \\
f_{l m n, \mathrm{TE}} & =\frac{c}{2} \sqrt{\left(\frac{\chi_{l m}^{\prime}}{\pi R}\right)^{2}+\left(\frac{n}{L}\right)^{2}}
\end{aligned}
$$

where the details to the derivation of (Eqs. 2.2.5a, 2.2.5b) are included in Appendix B. To test the ability of the computational model to simulate resonant cavity modes, the input frequency is fixed, and the radius and length are varied. The calculation results in a range of combinations of cavity dimensions which resonate for a specific input frequency $f_{0}$.

\subsubsection{Resonating cavity design procedure}

Set the resonant frequencies to a fixed input frequency $f_{l m n, \mathrm{TM}}=f_{0}$, or $f_{l m n, \mathrm{TE}}=f_{0}$ (depending on which type of mode profile is sought after). Calculate the combinations of radii $R$ and lengths $L$ which result in cavity resonances with input frequency $f_{0}$. There are two classes of resonances, the first (with $n=0$ ) result in radii where the cavity undergoes a $\mathrm{TM}_{\operatorname{lm} 0}$ resonance, these are considered 'longitudinal' cavity resonances.

$$
R=\frac{c \chi_{l m}}{2 \pi f_{0}}
$$

where $l \geq 0$ and $m \geq 1$. The other class of modes are the cases where $n \geq 1$, in which the mode has periodic structure along the length of the resonant 
cavity. The second class of resonances are associated with the following set of solutions;

$$
\begin{array}{ll}
L=\frac{n}{\sqrt{\left(\frac{2 f_{0}}{c}\right)^{2}-\left(\frac{\chi_{l m}}{\pi R}\right)^{2}}} & \text { TM mode } \\
L=\frac{n}{\sqrt{\left(\frac{2 f_{0}}{c}\right)^{2}-\left(\frac{\chi_{l m}^{\prime}}{\pi R}\right)^{2}}} & \text { TE mode }
\end{array}
$$

where $l \geq 0, m, n \geq 1$. From the equations above, the set of resonant cavity radii and lengths can be visualised in Figure 2.3. This set of analytical solutions provides a useful benchmark validation test for the COMSOL finite element model built in this work. These cavity dimensions deliver large average resonant electromagnetic field densities when compared to non-resonant cavity dimensions.

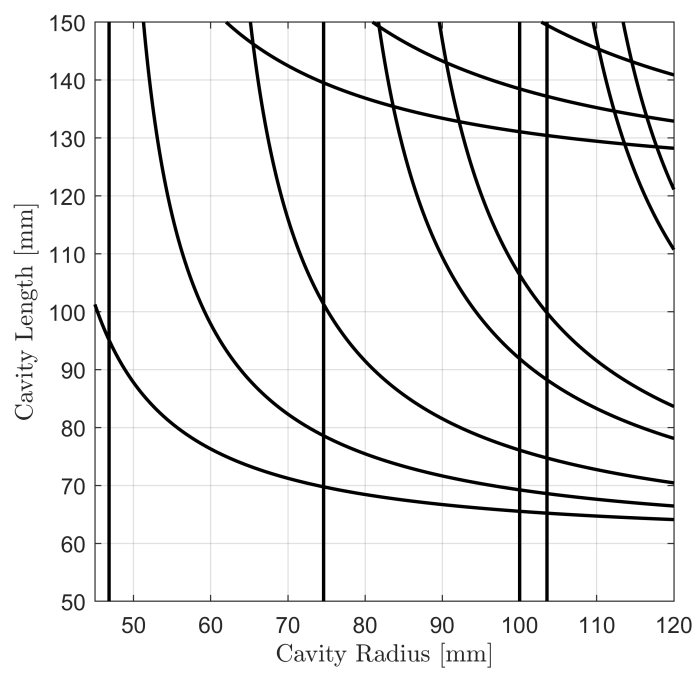

Figure 2.3: Plot showing combinations of cylindrical cavity radii and lengths which produce electromagnetic resonant modes at fixed frequency of $2.45 \mathrm{GHz}$. The lines (combinations of resonant cavity dimensions) are calculated directly from (Eqs. 2.2.6, 2.2.7a, 2.2.7b). The curves in this figure are exactly analogous for the right-handed resonance cylinder found in [38]. 


\subsection{Thermal losses}

As the ironsand in the cavity is heated due to the absorption of microwave energy, that thermal energy begins to distribute throughout the cavity. There are a variety of processes for this thermal energy to distribute within the microwave furnace, reducing the highest achievable temperature in the cavity. To mitigate the thermal losses, and maximise the temperature of the ironsand in the cavity, the most significant forms of thermal losses are reviewed; radiation, conduction and convection. For each type of loss; the concept is briefly introduced and reviewed. Specific heat loss mitigation strategies specific to the design of the furnace are discussed in Chapter 4.

\section{Radiation}

The most relevant type of heat loss in this work at high temperatures $\left(>600{ }^{\circ} \mathrm{C}\right)$, is thermal radiation. Heat energy loss through thermal radiation occurs when a material has a higher temperature than its environment, though is most significant at large temperature differences $\left(\Delta T>300^{\circ} \mathrm{C}\right)$. The heated material emits power in the form of electromagnetic radiation to its surroundings [43]. An ideal radiating body is known as a blackbody radiator and emits thermal radiation proportional to the fourth power of its temperature according to the Planck radiation law. The total power emission of a hot body at a given temperature with respect to its environment at temperature $T_{0}$ is

$$
P_{\text {rad }}=\epsilon(T, f) \sigma_{B} A\left(T^{4}-T_{0}^{4}\right)
$$

with the Stefan-Boltzmann constant $\sigma_{B}=5.670 \times 10^{-8} \mathrm{Wm}^{-2} \mathrm{~K}^{-4}$, surface area $A$ and emissivity $\epsilon$. The emissivity is a frequency and temperature dependent function $\epsilon(T, f)$ which describes how a material radiates power, and it ranges from 0 to 1 for any combination of $f$ and $T$. A value of $\epsilon\left(f_{0}, T\right)=0$ for a specific frequency means the material does not emit radiation of that frequency $f_{0}$. An emissivity of $\epsilon\left(f_{0}, T\right)=1$ means that the material ideally emits radiation at that specific frequency $f_{0}$. Blackbody radiation is considered to occur in a vacuum, so $c=f \lambda$. A perfect blackbody $\epsilon(\lambda, T)=1$ is a material that emits an ideal blackbody thermal radiation spectrum, given by

$$
B(\lambda, T)=\frac{2 h c^{2}}{\lambda^{5}} \frac{1}{e^{\frac{h c}{\lambda k_{B} T}}-1}
$$


where $B(\lambda, T)$ is the spectral radiance, $h=6.626 \times 10^{-34} \mathrm{Js}^{-1}$ is Planck's constant and $k_{B}=1.381 \times 10^{-23} \mathrm{JK}^{-1}$ is Boltzmann's constant. The radiative power loss term (Eq. 2.3.1) contains a $T^{4}$ term, which means it becomes the dominating type of thermal loss in the system at high temperatures relative to its surroundings ${ }^{10}$. This type of thermal loss mechanism can be reduced by raising the temperature of the ambient air and neighbouring materials (i.e. raising $\left.T_{0}\right)$.

\section{Conduction}

The next most important type of thermal loss mechanism is through conduction. Conductive losses describe how heat energy is transferred by diffusive means through a thermally conductive medium. The heat equation is a partial differential equation which models this, and is written as

$$
\frac{\partial U}{\partial t}=\frac{k_{t}}{\rho c_{p}} \nabla^{2} U
$$

with heat energy $U(\vec{r}, t)$ diffusing throughout the thermally conducting medium, where $k_{t}$ is the thermal conductivity, $\rho$ is the material density and $c_{p}$ is the material heat capacity. In this work, each of these parameters are scalar functions, generally varying only with respect to temperature. This type of loss can be reduced by choosing materials which have a low thermal conductivity. Generally, when analytically solved the solution to this type of differential equation is a Fourier series [44].

\section{Convection}

Convective heat loss occurs during the flow of a gas over a hot surface [45], with the gas constituents randomly colliding with the surface, and rebounding with added kinetic energy from the thermal energy of the hot surface. This extra kinetic energy adds to the thermal energy of the gas, and is then transported away from the surface by the gas. Any gas passing over a heated surface will therefore convectively cool that surface. At low temperatures, and in cases which have high gas flow rates, convective cooling can be the dominant cooling

\footnotetext{
${ }^{10}$ This is due to other types of thermal losses being proportional to lower powers of $\Delta T$.
} 
process with form

$$
P_{\text {conv }}=h A\left(T-T_{0}\right)
$$

where $A$ is the area, $T$ is the temperature of the hot object, $T_{0}$ is the temperature of the ambient gas, and $h$ the heat transfer coefficient of the interface material that is losing heat energy. This type of thermal loss can be mitigated by reducing the air (or other gas) flow rate over the hot material.

Using COMSOL the differential equations describing thermal transfer can be included along with electromagnetic effects. If this is correctly implemented (using correct material parameters), then the transient heating properties of the microwave furnace can be modelled.

\subsection{Using COMSOL Multiphysics}

Whilst analytical calculations can be used to find the propagating or resonating electromagnetic fields in specific idealised geometries, analytical solutions are not generally available for more complex geometries. Fortunately, today there are powerful FEM engines available which employ use of spatial discretisation and specified boundary conditions to solve partial differential equations in 3D space. By the existence and uniqueness theorems for fields with associated boundary conditions, the finite element solver produces a numerical solution for the unique field solution which meets the given boundary conditions. The FEM softawre that was used in this research is COMSOL Multiphysics. COMSOL is

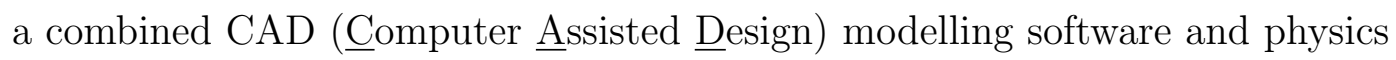
engine which allows the user to build a geometry, discretise it into a 'mesh grid' and calculate the partial differential equations of electromagnetism and thermal physics through every node throughout the mesh grid. A major advantage of COMSOL rests in its ability to couple multiple physics engines together into one self-consistent model. This allows the user to investigate systems where the thermal properties affect the electromagnetic performance of the system, and vice-versa. COMSOL enables the basic geometric properties of a microwave furnace to be modelled and optimised, without needing to build many furnaces through an experimental 'guess-and-check' approach. Since the mathematics describing electromagnetic and thermal behaviours are wellestablished, if the computational power of COMSOL is implemented properly 
its numerical solutions should closely approximate physical results.

\subsubsection{Introduction to FEM simulations}

The general method by which a problem is input into COMSOL is by first building a geometry with an inbuilt CAD software. In the case of a 3-dimensional system, the user defines a 3-dimensional mesh which appropriately represents the original geometry. To find the electric and thermal fields throughout the spatially discretised mesh, the boundary conditions for each field must be defined. From the existence and uniqueness theorems, this uniquely determines the full field solution within the specified geometry. Examples of boundary conditions are a perfectly conducting boundary for the electromagnetic field, or a fixed temperature boundary condition for the heat energy field. In the case of the electromagnetic field solver, COMSOL solves the partial differential equations of the electromagnetic field using multivariable functions (i.e. $\vec{F} \equiv \vec{F}(r, t)$ ) to find the quadratic finite differences of field values between neighbouring nodes in the mesh separated by distance $\Delta r$.

$$
\Delta \vec{F}=\frac{\partial \vec{F}}{\partial r} \Delta r+\frac{\partial^{2} \vec{F}}{\partial r^{2}} \Delta r^{2}
$$

This is the quadratic finite difference between two points in a vector field. Analogously, for the temperature field the Quadratic Lagrange discretisation method is employed by COMSOL to calculate the temperature field throughout the specified geometry.

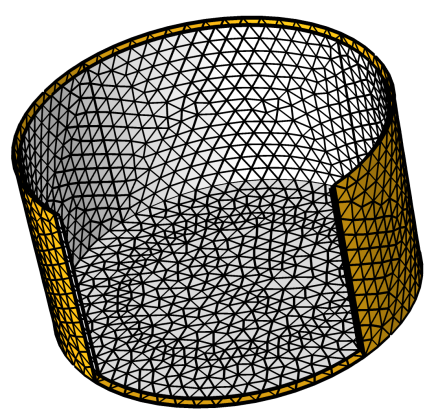

Figure 2.4: Example of tetrahedral meshing used in COMSOL Multiphysics for Figure 2.2. In this example, the dimensions of a cylindrical resonant cavity are $R=68 \mathrm{~mm}$ and $L=72 \mathrm{~mm}$.

The meshing shown in Figure 2.4 was used to produce a matching resonant cavity dimension profile as in Figure 2.3 for a fixed input frequency 
$f_{0}=2.45 \mathrm{GHz}$. COMSOL calculates the variation of the fields between all neighbouring mesh nodes with (Eq. 2.4.1). It thereby finds a near-unique field distribution within the system of predetermined boundary conditions. This process is repeatedly iterated so that the field variations are recalculated between points until the relative errors between each iteration fall below a preset error tolerance (generally set to $\leq 10^{-3}$ ). If large mesh spacing is used then the difference in field amplitude between those nodes is strongly affected by the large physical distance between them, so any differential rates of change $\partial^{2} \vec{F} / \partial r^{2}$ (or $\partial \vec{F} / \partial r$ ) will not be properly represented. This will result in inaccurate modelling results. If the node spacing is significantly reduced, then spatial accuracy of the solution will be increased, at the expense of greatly increased computation time. This is because increasing the number of mesh nodes increases the degrees of freedom (and hence the complexity of calculation) of the computation. This describes the importance of choosing the appropriate mesh density to give useful modelling results in a computationally efficient manner. In all following models in this thesis, mesh spacing which were less than or equal to one tenth of the wavelength of the microwaves in the system (i.e., $1.23 \mathrm{~cm}$ ). Later on in this thesis, the ironsand is modelled with a particularly dense mesh, as its real permittivity increases significantly near its Curie temperature, meaning that the wavelength of the $2.45 \mathrm{GHz}$ microwaves reduces greatly, requiring a higher mesh density for accurate simulation.

\subsubsection{Equations of COMSOL Multiphysics}

COMSOL uses partial differential equations to model the dynamics of systems [46]. When solving an electromagnetic system, the governing partial differential equation used is

$$
\nabla \times \frac{1}{\mu_{r}}(\nabla \times \vec{E})-k_{0}^{2}\left(\varepsilon_{r}-\frac{i \sigma}{\omega \varepsilon_{0}}\right) \vec{E}=0
$$

where $k_{0}$ is the free space wavenumber, $\sigma$ is the electrical conductivity, $\varepsilon_{r}$ is the complex relative permittivity and $\mu_{r}$ is the complex relative permeability. The magnetic field $\vec{H}$ is uniquely derived from the electric field $\vec{E}$ via Maxwell's equations (Eqs. 2.1.1a, 2.1.1b, 2.1.1c, 2.1.1d). The most common type of boundary condition used in this work was the impedance boundary condition, where the electric field only barely penetrates into a non-perfectly conducting material. This is used to reduce the domain size of calculation, 
thus increasing computational speed while still incorporating small conductive losses at those boundaries. The impedance boundary condition was used at all copper boundaries. The impedance boundary condition as used in COMSOL is written as

$$
\sqrt{\frac{\mu_{0} \mu_{r}}{\varepsilon_{0} \varepsilon_{r}-i \sigma / \omega}} \hat{n} \times \vec{H}+\vec{E}-(\hat{n} \cdot \vec{E}) \hat{n}=0
$$

with $\hat{n}$ the vector perpendicular to the interface, under the assumption that there are no other source electric fields. The other type of boundary condition used in this work is a 'Port' boundary condition, this is where electromagnetic energy enters or exits the model. In this work, input powers between $500 \mathrm{~W}$ and $1000 \mathrm{~W}$ are used to model the power entering the microwave cavity from the transmission line. The unique electric field $\vec{E}$ is calculated from (Eq. 2.4.2), under the impedance boundary conditions (Eq. 2.4.3) of the specified geometry, with an input Port delivering electromagnetic energy to the system, while writing the finite difference between locations as (Eq. 2.4.1). A full calculation of the electromagnetic field requires knowledge of the complexvalued permittivity $\varepsilon_{r}$ and complex valued permeability $\mu_{r}$ of all materials within the model.

Modelling the thermal evolution of the system during heating, also requires inclusion of Heat Transfer in Solids described in [46]. COMSOL is specifically built to couple various partial differential equations, making it an excellent software for modelling steady-state and transient microwave heated systems. Both transient and steady state models can be solved independently. The transient model calculates the resulting microwave heating over time $t$ starting from some initial condition. When no materials are moving in the model, the heat equation (Eq. 2.3.3) in the transient system is written

$$
\begin{gathered}
\rho c_{p} \frac{\partial T}{\partial t}+\nabla \cdot \vec{q}=Q_{e} \\
\vec{q}=-k \nabla T
\end{gathered}
$$

with $\vec{q}$ the heat flux vector and $Q_{e}$ the sum of electromagnetic losses in the system; $Q_{e}=Q_{r h}+Q_{m l}$. The resistive heating $Q_{r h}$ and magnetic losses $Q_{m l}$ 
are written as

$$
\begin{aligned}
Q_{r h} & =\frac{1}{2} \Re\left\{\vec{J} \cdot \vec{E}^{*}\right\} \\
Q_{m l} & =\frac{1}{2} \Re\left\{i \omega \vec{B} \cdot \vec{H}^{*}\right\}
\end{aligned}
$$

from the COMSOL RF Module User's guide [46], which are placed into (Eq. 2.4.4a) to calculate the thermal evolution in the model where materials absorb microwave power. Therefore, the thermal conductivity $k_{t}$, heat capacity $c_{p}$ and density $\rho$ for each material also need to be known. In addition; at high temperatures, materials thermally radiate. The Surface-to-Ambient boundary condition is used

$$
-\hat{n} \cdot \vec{q}=\epsilon_{T} \sigma\left(T_{0}^{4}-T^{4}\right)
$$

where $\epsilon_{T}$ is the total emitted electromagnetic power of a material at temperature $T$ at that boundary, relative to a blackbody, known as the emittance of the material. The Surface-to-Ambient boundary condition uses the normal surface vector $\hat{n}$ and calculates the heat flux vector $\vec{q}$.

The steady state modelling procedure case calculates directly the $\partial_{t} T=0$ condition for the heat equation (Eqs. 2.4.4a, 2.4.4b) at each point in the mesh grid. As the material properties change as functions of temperature (over the large temperature range in this work), and because thermal radiation is a highly nonlinear process, this computation requires repeated recalculation of the electromagnetic and thermal fields between each solver step. This highlights the interplay between the different modelling interfaces in COMSOL. Material properties in this research are generally considered temperature-dependent, linear, time-invariant and isotropic scalar functions. This thermal variation of parameters adds complexity to the model, so an understanding of material properties between room temperature and $1000^{\circ} \mathrm{C}$ was required for a working computational model, as this was the approximate upper bound for $T$ in this work. Unfortunately, not all the desired material properties are known across this large temperature range, so assumptions about material properties must be made outside the known ranges which were taken from literature. Details on the initially used material properties from literature are included at the end of this chapter. 


\subsubsection{Simple resonating cavity}

To check the validity of the COMSOL RF module used in this work, a model of the cylindrical cavity (Figure 2.4) was solved, and these results compared with analytical solutions from Figure 2.3. This cavity has radius $R$, length $L$, the filling material in the cavity is air, and the outside boundaries are assumed to be perfectly conducting.

The total energy in the electromagnetic field resonance for a given set of radii $R \in(45,120) \mathrm{mm}$ and lengths $L \in(50,150) \mathrm{mm}$ at a fixed frequency $\left(f_{0}=2.45 \mathrm{GHz}\right)$ was calculated in COMSOL as a benchmark test. This range of parameters covers all of the low order TM and TE modes. At large cavity dimensions there are many modes that do not have large central field maxima. This is due to radial and longitudinal repetition in mode structure, weakening the central intensity of resonances. Thus, the search region for the resonant modes is thereby restricted to the aforementioned ranges. The total electromagnetic energy $Q_{E M}(R, L)$ for a combination of cavity dimensions $R$ and $L$ was evaluated by integrating the electromagnetic field density [36] in the cavity.

$$
Q_{E M}(R, L)=\int_{r=0}^{r=R} \int_{l=0}^{l=L} \int_{\varphi=0}^{\varphi=2 \pi} U_{E M}(r, \varphi, l) R d r d \varphi d l
$$

Resonant cavity modes can be identified with high electromagnetic field intensities. Combinations of cavity radii and length which are associated with a resonant mode can be found by FEM simulation, and compared to the analytical result. To compare the quality of resonances in different sized cavities, the average electromagnetic field energy density throughout the cavity is calculated for each combination of dimensions with

$$
U_{E M}(R, L)=\frac{Q_{E M}(R, L)}{V(R, L)}
$$

where $Q_{E M}(R, L)$ from (Eq. 2.4.7). Performing this evaluation results in good agreement with the analytical curves from Figure 2.3, albeit at a low 'spatial resolution' ( $2 \mathrm{~mm}$ ) due to the step-sizes and mesh size densities used during this modelling procedure. The mesh element size was restricted between $0.2 \%$ and $5 \%$ of wavelength in each medium for this work ${ }^{11}$. This

\footnotetext{
${ }^{11}$ In dielectrics, the wavelength of light reduces proportional to the refractive index $n=\Re\left\{\sqrt{\varepsilon_{r} \mu_{r}}\right\}$ of the material via $\lambda^{\prime}=\lambda / n$
} 
modelled parameter sweep with average field density evaluation (Eq. 2.4.8) took approximately 2 days.

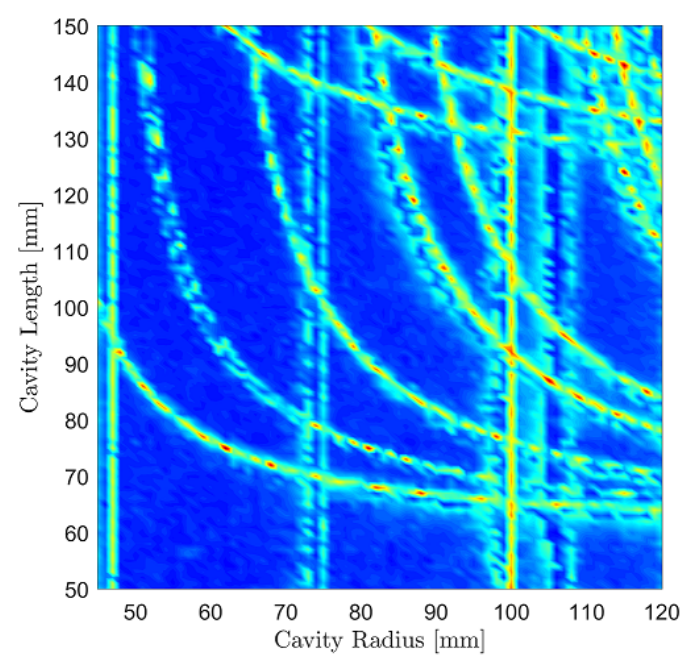

Figure 2.5: The simulated resonance curves as evaluated via (Eq. 2.4.8) for each combination of $R \in(45,120) \mathrm{mm}$ and $L \in(50,150) \mathrm{mm}$.

Figure 2.5 shows the results of the COMSOL model calculations. The same resonance curves appear as were obtained from the analytical calculations using (Eqs. 2.2.6, 2.2.7a, 2.2.7b) and shown in Figure 2.3. This provided confidence that the COMSOL model built for this work reliably reproduces known electromagnetic solutions (given a sufficiently resolved mesh grid for the model).

\subsection{Model material properties}

This section describes the material properties used as the starting point in the computational model in this research. The material properties of copper from which the cavity is built is taken from internally preset COMSOL Multiphysics 5.6 materials [46]. The room temperature electrical conductivity of copper is $5.87 \times 10^{7} \mathrm{~S} / \mathrm{m}$, thermal conductivity $400 \mathrm{~W} / \mathrm{mK}$, density $8.96 \mathrm{~g} / \mathrm{cm}^{3}$ and heat capacity $385 \mathrm{~J} / \mathrm{kgK}$, taken from the material library within COMSOL. 


\subsubsection{Boron Nitride}

BN ( Boron Nitride) ${ }^{12}$ is a well studied microwave-transparent ceramic material $[47,48]$ with high thermal stability, chemical inertness and high thermal conductivity [49]. BN tubes pictured in Figure 2.6 were used to contain the ironsand within the microwave cavity. The large temperature range material properties of BN were essential to know for accurate computational modelling, and are shown in Figure 2.7. The magnetic properties of BN are considered to be unitary over the entire temperature range of interest, (i.e. $\mu_{r}(T)=1-0 i$ ). The relevant material properties of $\mathrm{BN}$ are therefore; heat capacity $c_{p}$, thermal conductivity $k_{t}$, emittance $\epsilon_{T}$ and relative permittivity $\varepsilon_{r}$.

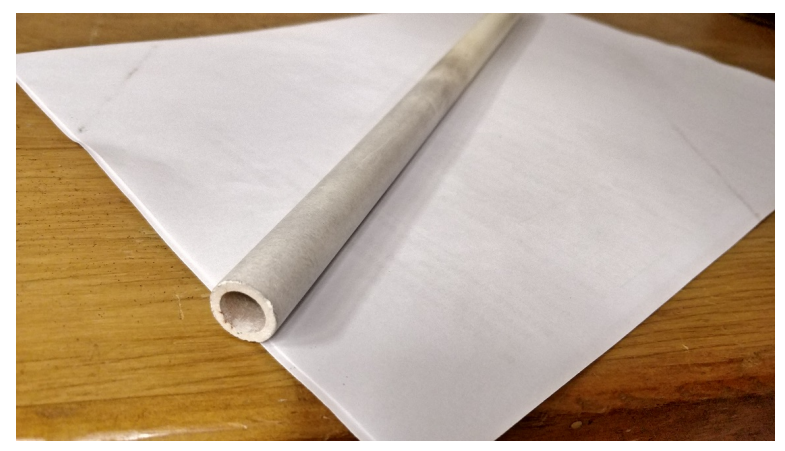

Figure 2.6: Picture of BN rod-turned-crucible used in this research. The drilled hole is $100 \mathrm{~mm}$ long and has a diameter of $10 \mathrm{~mm}$. The tube diameter is $15 \mathrm{~mm}$ and its length is $300 \mathrm{~mm}$

The density was measured to be $2.00 \pm 0.01 \mathrm{~g} / \mathrm{cm}^{3}$ by scale measurement. The specific type of BN used in this research was hot-pressed hexagonal Boron Nitride produced by Innovacera, pictured in Figure 2.6. The temperature dependent thermal conductivity and heat capacity of BN is taken from [50], and data for the emittance of BN was obtained from [51].

\subsubsection{Insulation fibreboard}

The largest volume of material within the microwave cavity in this research is the thermal insulation fibreboard. The fibreboard used in this work is an alumina fibreboard, specifically AL-25/1700 compromised of $80 \% \mathrm{Al}_{2} \mathrm{O}_{3}$, and $20 \% \mathrm{SiO}_{2}$ [52]. Annuli disks cut to fit the cavity are shown in Figure 2.8.

\footnotetext{
${ }^{12}$ Colloquially known as 'white graphite' due to its similar mechanical properties
} 

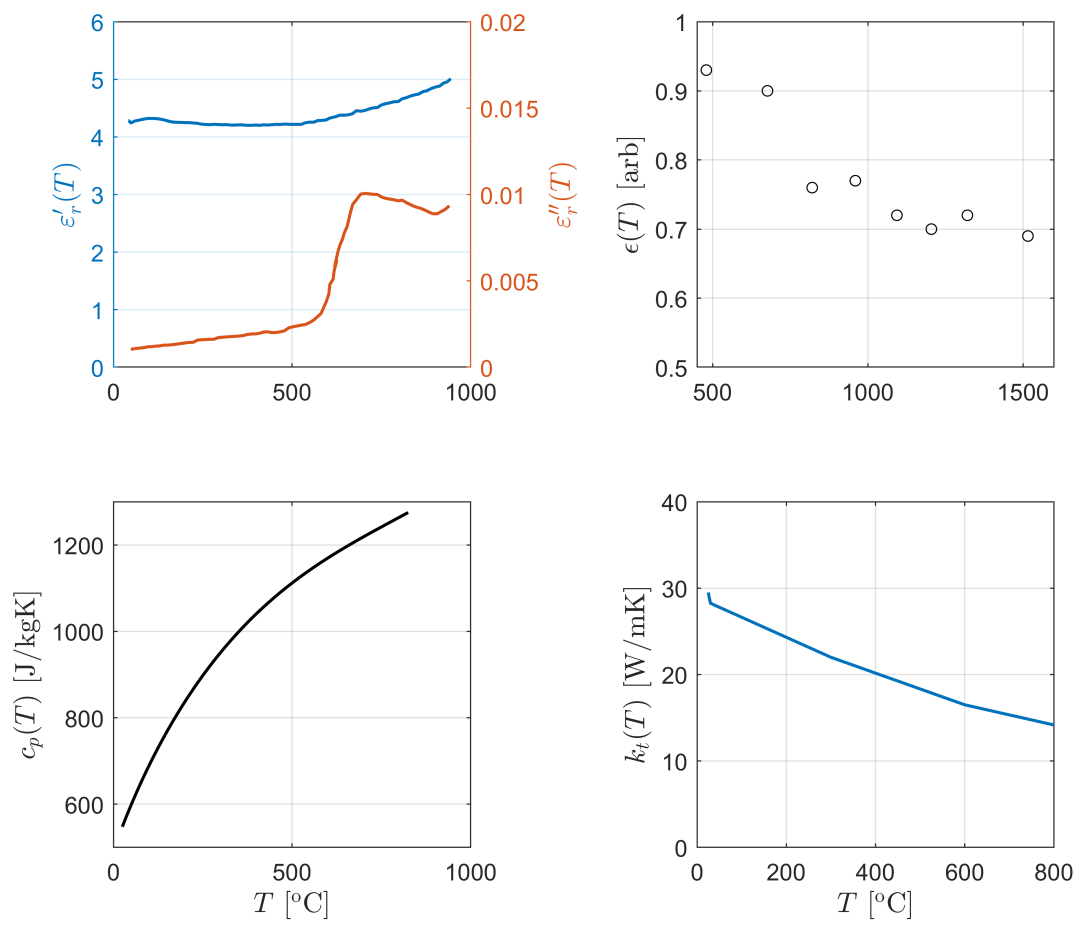

Figure 2.7: Relevant temperature dependent material properties of BN used in modelling calculations (Top left; complex permittivity $\varepsilon_{r}^{\prime}(T), \varepsilon_{r}^{\prime \prime}(T)$, top right; emittance $\epsilon(T)$, bottom left; heat capacity $c_{p}(T)$, bottom right; thermal conductivity $\left.k_{t}(T)\right)$.

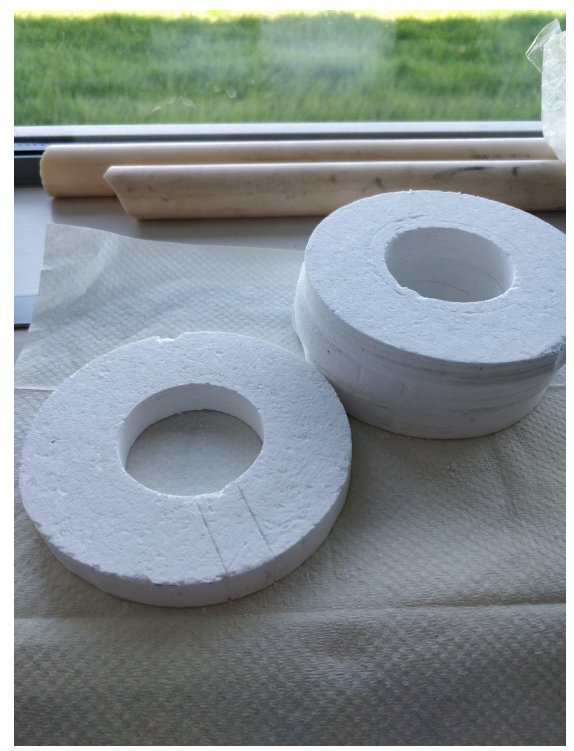

Figure 2.8: Half-inch thick AL-25/1700 alumina fibreboard annuli cut-outs stacked on paper towel before placement into microwave cavity. 
A fibreboard was chosen over loose fibre insulation due to the high mechanical stability and uniformity of the fibreboard material, which ensured high experimental repeatability. The insulation has a low thermal conductivity and low real and imaginary permittivity at $2.45 \mathrm{GHz}$. The quoted dielectric loss at $2.45 \mathrm{GHz}$ is $\varepsilon_{r}^{\prime \prime} / \varepsilon_{r}<0.01$, and the real permittivity is $\varepsilon_{r}=1.4$ [53]. Similar fibreboards from this supplier have negligible permittivity variations from room temperature to $1000^{\circ} \mathrm{C}$, so the same was assumed for the AL-25/1700 fibreboards. The manufacturer's specified density is $0.4 \mathrm{~g} / \mathrm{cm}^{3}$, and the heat capacity is $1047 \mathrm{~J} / \mathrm{kgK}$. In addition, the specified thermal conductivity of the fibreboard is $0.20 \mathrm{~W} / \mathrm{mK}$ at $400^{\circ} \mathrm{C}$ and linearly increases to $0.46 \mathrm{~W} / \mathrm{mK}$ at $1600{ }^{\circ} \mathrm{C}$ [52]. Board thicknesses of half an inch were purchased and cut into annuli (inner radius $22.5 \mathrm{~mm}$ and outer radius $50 \mathrm{~mm}$ ) to be centrally stacked in the cavity. The inclusion of thermal insulation reduces radiative losses, however affects the microwave resonance in the cavity due to the increase in $\varepsilon_{r}^{\prime}$ [36]. This was modelled using FE, and the effect thereof is shown in Chapter 3.

\subsubsection{Titanomagnetite ironsand}

The crystal structure of TTM is similar to that of standard magnetite [18, 54], making magnetite a good starting point for computational modelling of the TTM ironsand. The simulations in this thesis model ironsand as a 'solid' bulk material, and thus its properties scale with relative density due to the packing factor of the ironsand, relative to its bulk counterpart. The particle size of the ironsand used in this work was generally between $90 \mu \mathrm{m}$ and $250 \mu \mathrm{m}$ [22]. The density of the raw TTM ironsand used in this research was measured to be $2.53 \pm 0.02 \mathrm{~g} / \mathrm{cm}^{3}$. The ironsand used in this work is pictured in Figure 2.9

\section{Relative density}

The density measurement of the ironsand was done by measuring the weight difference of an alumina crucible with known volume before and after filling with TTM ironsand. Under the assumption that the density of bulk TTM is approximately identical to that of magnetite $\left(5.03 \mathrm{~g} / \mathrm{cm}^{3}\right)$, the relative density of TTM in this work was therefore $50.4 \%$. The relative density of the magnetite powder samples used in [24] was $62.9 \%$. 


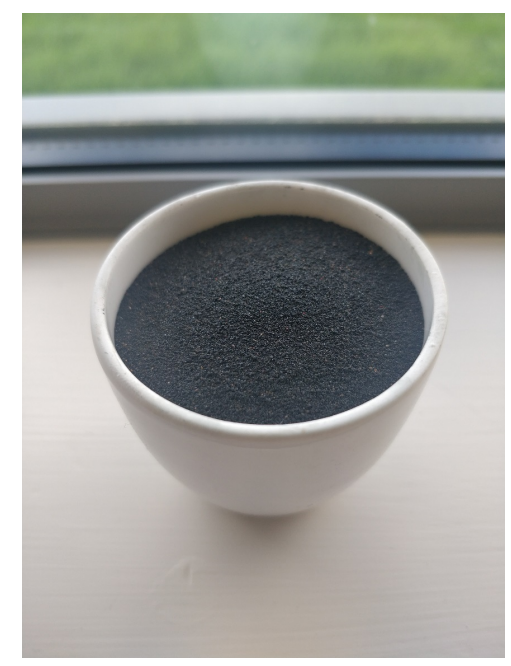

Figure 2.9: Cup of TTM ironsand, exemplifying the black colour of the ironsand. The lighter specks in this figure are impurities in the ironsand. This powder was filled into the BN tube from Figure 2.6 for experiments.

\section{Electric and magnetic properties}

The magnitude of the complex permittivity and permeability was scaled by the ratio of relative densities, i.e. by 0.8 . Reducing the permittivity and permittivity by this scale factor was done to describe the variation in electromagnetic properties as a function of material density. When directly comparing magnetite and titanomagnetite: the titanium species replacing the iron species in $\approx 8 \%$ reduces the electromagnetic response of the bulk material. Titanium is also a lighter element than iron, with atomic weights $47.867 \mathrm{amu}$ and $55.845 \mathrm{amu}$ respectively [55]. The Curie temperature is the temperature at which materials undergo a phase change in their magnetic properties [56], and for TTM this is $430^{\circ} \mathrm{C}$ and results in the loss of permanent magnetisation of TTM. This was measured in-house by Mimi Kurian on a PPMS (Physical Property

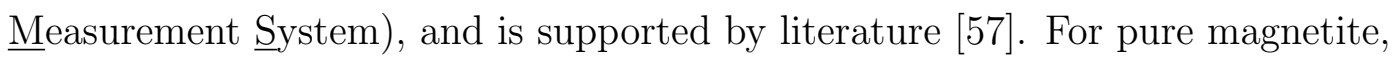
this temperature is $\approx 575^{\circ} \mathrm{C}[58,59]$. Thus, the electromagnetic and thermal properties are linearly off-set in temperature by $145^{\circ} \mathrm{C}$. From Figure 2.10, it can be seen that $\mu_{r}^{\prime}$ and $\mu_{r}^{\prime \prime}$ reduce monotonically above $400^{\circ} \mathrm{C}$. Magnetite is a good absorber of magnetic microwave energy at low temperatures. It is also noted that the real permittivity $\varepsilon_{r}^{\prime}$ also reduces drastically beyond $500{ }^{\circ} \mathrm{C}$. The imaginary permittivity $\varepsilon_{r}^{\prime \prime}$ begins low, and begins to significantly increase at above $300{ }^{\circ} \mathrm{C}$, due to the contribution of significant conductive losses at high 
temperatures.
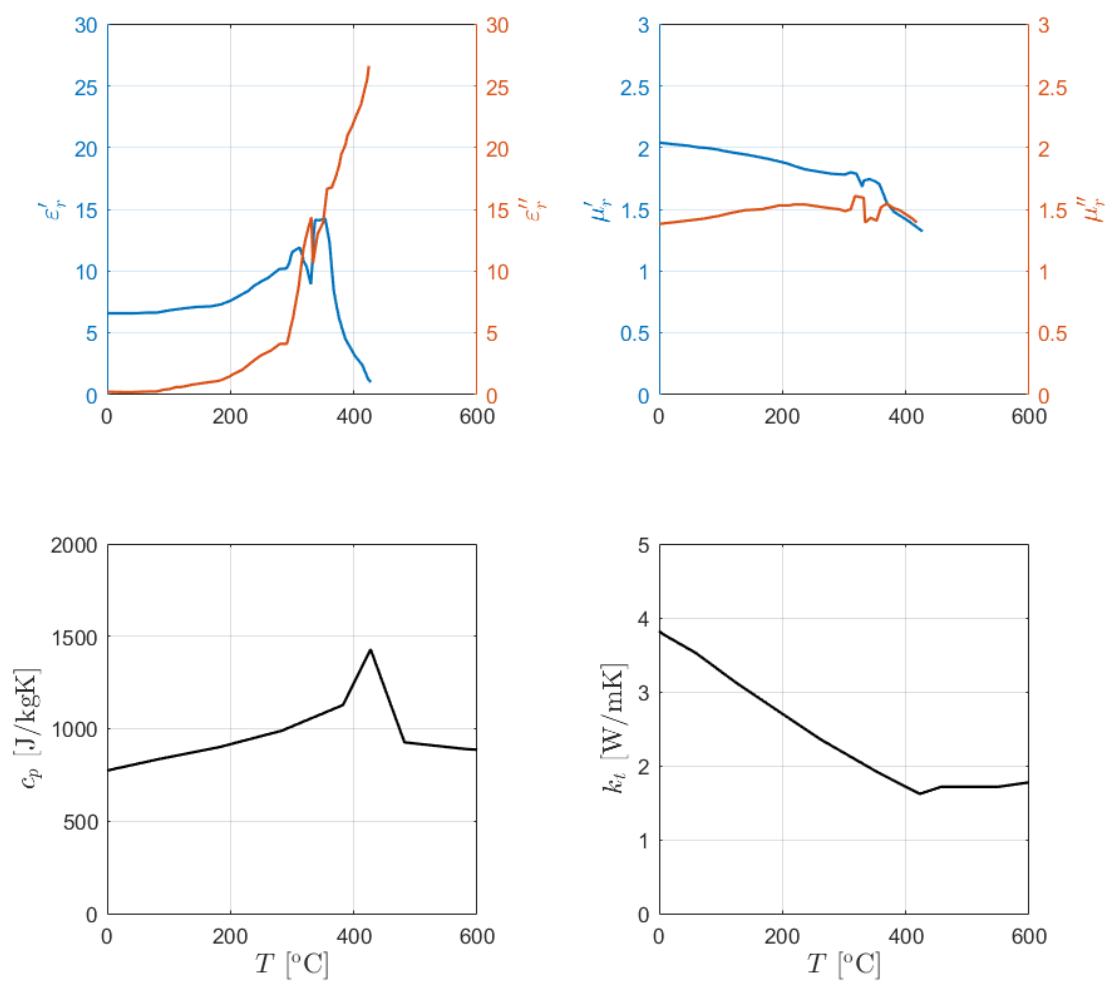

Figure 2.10: Temperature dependent values used in the initial model for TTM ironsand adapted from literature [24,60] values for magnetite (Top left; complex permittivity $\varepsilon_{r}^{\prime}(T), \varepsilon_{r}^{\prime \prime}(T)$, top right; complex permeability $\mu_{r}^{\prime}(T), \mu_{r}^{\prime \prime}(T)$, bottom left; heat capacity $c_{p}(T)$, bottom right; thermal conductivity $k_{t}(T)$ ).

Due to the large temperature range reached by the TTM in this research, material parameter extrapolation must be done for the model to be useful in the high temperature regime. The magnetic permeability was expected to reduce rapidly to unitary values beyond the measurement range in Figure 2.10, so the initial finite element model assumes that the reduction in permeability is linearly extrapolated $\left(\mu_{r}=1-0 i\right)$ above $430{ }^{\circ} \mathrm{C}^{13}$. This was a reasonable approximation to make, as due to the high temperatures reached by the system, the thermal energy in the system entirely overwhelms the energy stored in the magnetic ordering within the ironsand. The same was true for the real permittivity $\varepsilon_{r}^{\prime}$,

\footnotetext{
${ }^{13}$ If the permeability is set to unitary values at and above the Curie temperature $T_{C}$, this would produce errors in transient simulations. This is due to significantly varying magnetic microwave properties from one point in the mesh grid to a neighbouring one, resulting in non-converging solver steps around $T_{C}$.
} 
where values were linearly extrapolated beyond the measurement range until $\varepsilon_{r}^{\prime}=1$ was reached.

The dielectric loss at high temperatures was also expected to reduce to near 0 , as almost all electromagnetic energy is transferred to the charge carriers due to direct and indirect band transitions of the charge carriers [61]. Magnetite is however a complex semiconductor, with a very narrow band gap of $0.1 \mathrm{eV}$ [62]. Semiconductors with narrow band gaps are also known as semi-metals [63]. The conductivity of metals as their temperature reduces due to increased phonon scattering $[37,64]$. In addition to this, it is also a p-type and n-type semiconductor [62], further increasing the complexity of the material's temperature-dependent conductivity. Due to the complex nature of the conductivity variation of TTM (and its lack of research in literature), writing its conductivity $\sigma(T)$ as an analytical function to include in COMSOL presents great difficulty. Thus, the literature values for the permittivity and permeability are linearly extrapolated beyond the measurement range in Figure 2.10 to minimise the amount of assumptions required to predict a more detailed high temperature conductivity variation.

\section{Thermal properties}

The thermal properties for the ironsand used in modelling calculations are taken from [60]. The heat capacity is off-set in temperature such that the peak heat capacity matches the temperature at which the permittivity and permeability drastically change (near $T \approx 430^{\circ} \mathrm{C}$ ). The same temperature off-set (due to the differing Curie temperature between pure magnetite and the ironsand used in this work) was done for the literature values of thermal conductivity and heat capacity. 


\section{Chapter 3}

\section{Initial prototype furnace modelling}

This chapter details the process of design development and initial FEM simulation of the prototype cavity. Firstly, the advantages of a cylindrical geometry, over rectangular and spherical microwave cavity types is analysed. Then, the fundamental design for a cylindrical microwave cavity is introduced, and methods for implementation of various design requirements for safe operation and ability to measure various parameters are discussed. Then, these designs are implemented in initial FEM simulations. A basic parametric sweep of cavity dimensions is carried out to find the optimal combinations of cavity dimensions to maximise power transfer rates to the TTM ironsand in the cavity.

After the cavity dimensions are determined, the resonance performance of the cavity is tested against the inclusion of thermal insulation. This chapter finishes with the starting point for FEM investigation in Chapter 5, with experimental results from experimental methods developed in Chapter 4.

\subsection{Cavity geometry and design criteria}

The cavity in this research was built with the continuous processing of TTM ironsand in mind. The aim of this thesis is the precursor to that continuous processing phase of research, where the microwave heating of static TTM ironsand must be understood both experimentally and computationally. The microwave cavity in this work was chosen to be of the cylindrical geometry. The cylindrical geometry is used as the TTM ironsand container material BN to be used in further research is cylindrical in nature. The computational results from 
Figure 2.5 inform the combination of cavity dimensions which result in various potential TE and TM resonances. The exact design requirement for which the empty cavity was optimised, was to have a centralised electromagnetic field mode structure to maximise power transfer to a BN tube filled with TTM ironsand centred in the cavity. This could be achieved if a $\mathrm{TM}_{010}$ or $\mathrm{TE}_{111}$ mode was chosen as the resonant mode type of the cavity. A range of modifications to the model in Figure 2.2 needed be made to engineer a cavity design which functioned as a single-mode microwave furnace. The cavity would also require a design which could yield refinements to a computational model through experimental measurements.

- Firstly, an input waveguide was added to the cavity, and since $2.45 \mathrm{GHz}$ microwave power is used, a WR340 input waveguide [39] was used. This affected the cavity's resonance profile, and therefore the performance of that resonant mode. The effect of adding the WR340 waveguide to the cavity depended on the input orientation of that waveguide, and the cavity dimensions

- Secondly, as the ironsand must be passed through the cavity through a single large BN tube, the cavity would need have large holes on both ends of the cavity. Employing BC (ㅌeyond-utoff) waveguides, it was possible to pass a large quartz and BN tubes through the cavity, while minimising microwave leakage.

- Thirdly, the temperature of the ironsand in the cavity needed to be measured with optical pyrometers, as thermocouple measurements were not possible in the microwave cavity without significantly affecting the heating dynamics of a microwave furnace [65]. Direct optical access to the ironsand-containing $\mathrm{BN}$ tube was therefore essential, meaning that small optical access holes needed to be added to the side of the microwave cavity.

To safely implement the second and third features, dual-purpose extra copper tubing was added to the holes at both ends of the cavity, and around the cavity. The design for the length of each type of tubing is discussed in Sections 3.3 for the second point, and Section 3.6 for the third. 


\subsection{Input waveguide orientation}

The WR340 waveguide supports mono-mode transfer of microwave energy in the $\mathrm{TE}_{10}$ mode, shown in Table 2.1. The magnetic field variation is restricted along the length and long side of the WR340 waveguide. Thus, if a horizontal input waveguide is used to deposit microwave energy in the cavity, the type of modes which are produced in the microwave cavity are primarily restricted to the cylindrical TM mode type. Similarly, if a vertical input waveguide orientation is used, cavity resonant mode types are restricted to the cylindrical TE mode type. The profile of each electromagnetic mode is unique, so the mode which best maximised the electromagnetic field mode in the centre of the cavity needed to be determined.

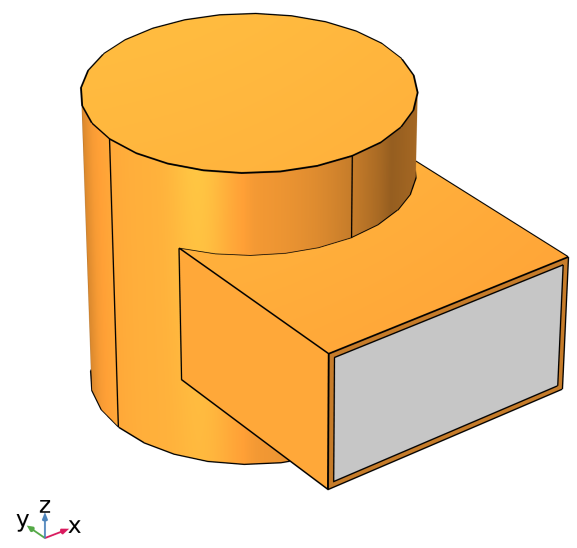

(a) Horizontal input WR340 waveguide

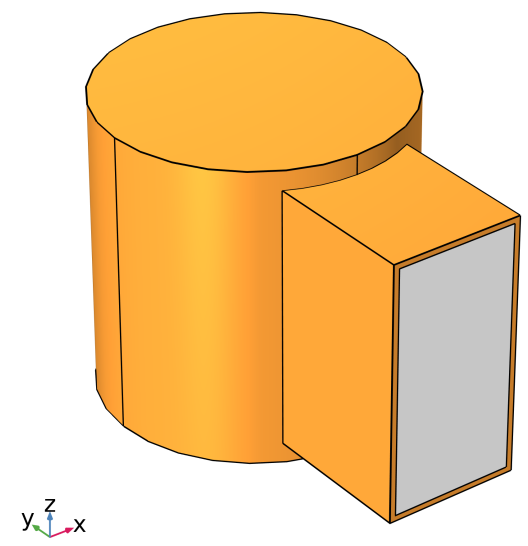

(b) Vertical input WR340 waveguide

Figure 3.1: Models of empty resonant cavities with input WR340 waveguides in (a) horizontal; and (b) vertical input orientations. The orange sections are the copper parts of the cavity, and grey corresponds to air.

The calculation for the suitable orientation of the WR340 waveguide is done after the $\mathrm{BC}$ waveguides are incorporated in the computational model, to reduce the range of parameters needed to be swept through. Due to the width of the WR340 waveguide, this resulted in combinations of minimum radii and lengths of the resonant cavities 3.1a and 3.1b shown in Table 3.1.

If an input waveguide orientation that is not horizontal or vertical is used, the resulting mode structure will be some superposition of both a TE and TM mode within the resonant cavity. This is not desired, as the purpose of the 


\begin{tabular}{||c|c|c||}
\hline WR340 input orientation & Cavity radius $[\mathrm{mm}]$ & Cavity length $[\mathrm{mm}]$ \\
\hline \hline Horizontal & 45 & 50 \\
\hline Vertical & 29 & 90 \\
\hline
\end{tabular}

Table 3.1: The smallest microwave cavity dimensions depending on input waveguide orientation with width $86.36 \mathrm{~mm}$ and height $43.18 \mathrm{~mm}$. These input orientations can be seen in Figures 3.1a and 3.1b.

cavity was to employ the field profile of a single centralised electromagnetic mode. The most standard electromagnetic field modes used in research are either the $\mathrm{TM}_{010}$ or $\mathrm{TE}_{111}$ mode $[66,67]$ to suit various other applications, e.g. particle accelerators [68-70], sputtering devices [71-73] and furnaces [74,75].

\subsection{Beyond-cutoff waveguides}

Since ironsand must be passed through the cavity in future work with the cavity designed in this work, two holes were added to the top and bottom of the cavity as a modification to Figure 2.2. For the sake of visualising the addition of the waveguides, the input waveguides from Figures 3.1a and 3.1b were omitted from 3.2 .

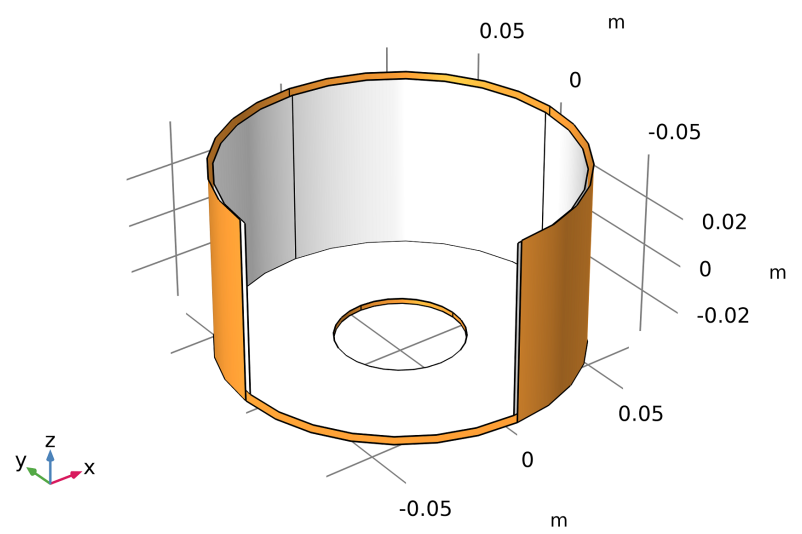

Figure 3.2: A cut-open view of an empty 3D resonant cavity model with radius $R$ and length $L$ built in COMSOL Multiphysics with two $50 \mathrm{~mm}$ (diameter) holes cut from the ends of the cavity (the top hole was hidden for the purposes of this picture).

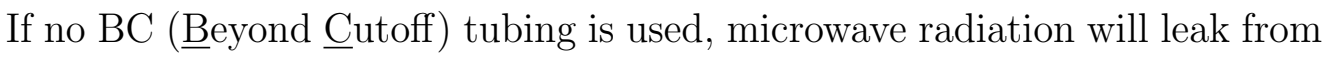


the cavity during usage. This is both a waste of electromagnetic energy, and a health risk for users near the cavity [76-79]. All propagating electromagnetic modes are determined by their cutoff frequencies, and the lowest cutoff frequency for a fixed cylindrical $\mathrm{BC}$ waveguide radius is the $\mathrm{TE}_{11}$ mode. In the case of a cylindrical BC waveguide with $R<35.68 \mathrm{~mm}$ as calculable from (Eq. 2.2.6), the cutoff frequencies of all TM and TE propagating modes are above the input frequency $\left(\omega_{0}=1.26 \times 10^{10} \mathrm{rad} / \mathrm{s}\right)$. This results in the electromagnetic wavenumber $k$ of that wave becoming purely imaginary, regardless of the mode in which the electromagnetic field propagates. No electromagnetic power can then be transferred along a BC waveguide, however some can 'leak' if that $\mathrm{BC}$ waveguide is too short to meaningfully attenuate the microwave energy. Therefore, BC waveguides can be used to minimise microwave leakage from an optically open cavity.

The lower limit on the radius of the $\mathrm{BC}$ waveguide was $20 \mathrm{~mm}$ as the furnace in this work was initially designed to have a large BN tube to be placed through it. The outer radius of that tube was $20 \mathrm{~mm}^{1}$. That larger tube was not used for the initial characterisation and modelling of the furnace. The addition of $\mathrm{BC}$ waveguides exponentially reduces microwave leakage intensity, with the exact form shown as (Eq. 3.3.1). The input angular frequency is $\omega_{0}=1.26 \times 10^{10} \mathrm{rad} / \mathrm{s}$. The lowest cutoff frequency is given by $\omega_{11}$, corresponding to the $\mathrm{TE}_{11}$ mode. The maximum radius $R$ for which the waveguide functions as a $\mathrm{BC}$ waveguide (where it does not propagate microwave energy) is found when the condition $\omega_{0}=\omega_{11}$ is set, is $R=35.86 \mathrm{~mm}$. The attenuation rate (per unit length) of a specific $\mathrm{BC}$ waveguide is written as $\mathrm{dB} / z$ where $z$ is the length of the $\mathrm{BC}$ waveguide. Solving (Eq. 3.3.1) for $\mathrm{dB} / z$ results in

$$
\frac{\mathrm{dB}}{z}=-\frac{10}{\ln (10)} \frac{2}{c} \sqrt{\omega_{11}^{2}-\omega_{0}^{2}}
$$

when the input frequency is below the cutoff frequency $\omega_{0}<\omega_{11}$. The details on this derivation are given in Appendix B. To have some clearance between the $\mathrm{BN}$ tube and $\mathrm{BC}$ waveguide, a $\mathrm{BC}$ waveguide radius of $25 \mathrm{~mm}$ was chosen, as it was an easy-to-procure dimension of Copper tubing. This was chosen as it was the smallest easily procurable Copper tubing that was larger than the BN tube. The decay rate as calculated from (Eq. 3.3.1) of a copper BC waveguide with $R=25 \mathrm{~mm}$ is $-4.59 \mathrm{~dB} / \mathrm{cm}$ (for $2.45 \mathrm{GHz}$ microwave power).

\footnotetext{
${ }^{1}$ The inner radius of the large $\mathrm{BN}$ tube was $11.5 \mathrm{~mm}$, and the tube was $1 \mathrm{~m}$ long.
} 
Thus, the intensity of electromagnetic field power decreases by a factor of 10 for each two centimetres added (if no BN tubing is present). The long term human safety limit for microwave radiation is $4939 \mathrm{~mW} / \mathrm{mm}^{2}$ [80], and this was used to infer suitable $\mathrm{BC}$ tube lengths. A $25 \mathrm{~mm}$ radius $\mathrm{BC}$ waveguide was decided to be suitable for reducing microwave leakage. Early on in the project, a BC waveguide length of $200 \mathrm{~mm}$ was chosen, thus reducing the leakage intensity by $\approx 100 \mathrm{~dB}$ relative to the resonant field intensity in the cavity. This would ensure that the $\mathrm{BC}$ waveguides effectively prevent microwave leakage from the cavity, even if a dielectric is added through the furnace (filling a BC waveguide with a dielectric will lower $\omega_{11}$ by factor $1 / \sqrt{\varepsilon_{r}^{\prime}}$ ). The initial design for the microwave cavity is then given in Figure 3.3.

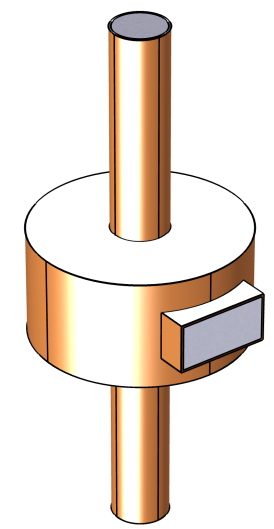

Figure 3.3: The closed initial design of the microwave furnace. A horizontal input WR340 waveguide is included (width $86.36 \mathrm{~mm}$ ) in addition to two BC waveguide tubes with radius $25 \mathrm{~mm}$ and length $200 \mathrm{~mm}$. The cavity dimensions for this figure are $R=68 \mathrm{~mm}$ and $L=72 \mathrm{~mm}$, but were not the final combination of dimensions chosen.

The dimensions of the $\mathrm{BC}$ waveguides were determined with radius $R=$ $25 \mathrm{~mm}$ and length $L=200 \mathrm{~mm}$. The next parameters to fix are the cavity radius, length (to select mode profile) and input waveguide orientation (to choose either TM or TE modes to occur in the cavity).

\subsection{Determining basic cavity dimensions}

Parameter sweeps were then done to visualise the resonance profile of various cavity dimensions, similarly to Figure 2.5. The minimum cavity dimensions 
for horizontal or vertical input waveguide orientations were given in Table 3.1. Low order electromagnetic modes generally have large central electromagnetic fields, so the same maximum cavity dimensions as in Figure 2.5 are used. In these models, an input power of $1000 \mathrm{~W}$ was used. The resonance 'quality' for an electromagnetic mode, given a specific combination of geometries, was determined via (Eqs. 2.4.7, 2.4.8) which calculate the average electromagnetic field energy density for each one.

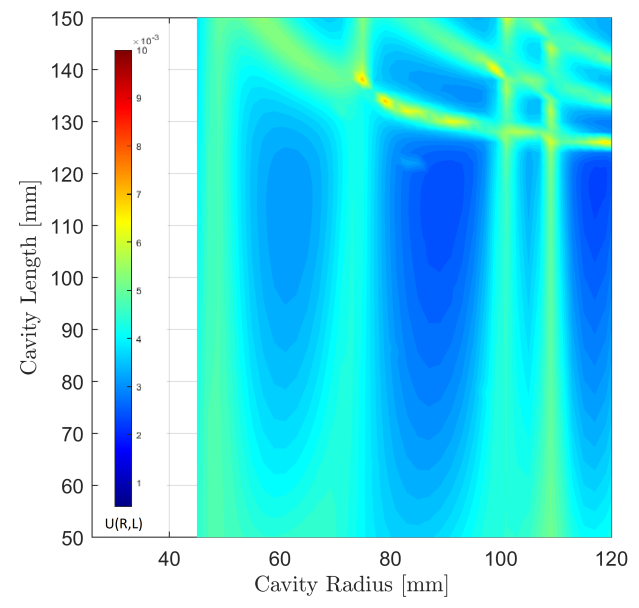

(a) Horizontal; $U_{E M}(R, L)$ evaluation

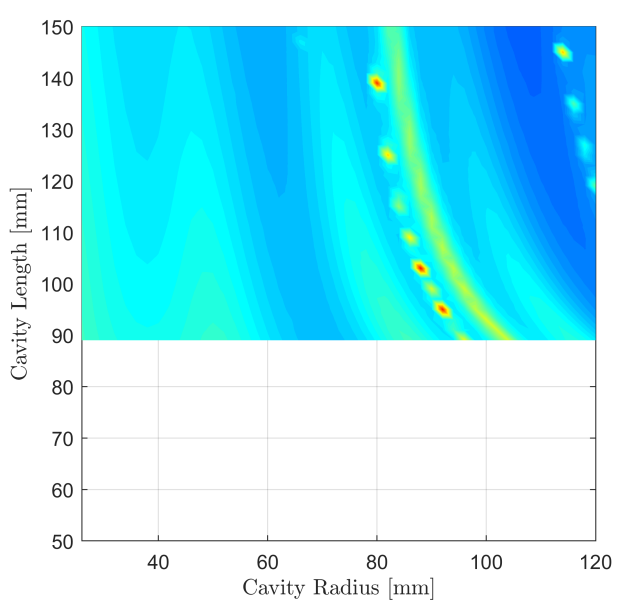

(b) Vertical; $U_{E M}(R, L)$ evaluation

Figure 3.4: Average EM energy density integrals (Eq. 2.4.8) for a variety of cavity radii and lengths with horizontal (left) and vertical (right) input waveguide. The model in Figure 3.3 was used for this simulation where the cavity length and radius are varied. Neither insulation or BN tubes were included in this parameter sweep, however the $50 \mathrm{~mm}$ diameter $\mathrm{BC}$ waveguide tubes were included.

Figures 3.4a and 3.4b demonstrate that the horizontal input waveguide results in TM resonances, and the vertical input waveguide results in TE resonances, distinguished from one another by the polarisation of the microwaves propagating through the WR340 waveguide. The 'vertical' resonances in Figure 3.4a are $\mathrm{TM}_{\operatorname{lm} 0}$ modes. These modes are of specific interest as they encode 'longitudinal' resonances, where the profile of the electromagnetic resonant field minimally varies along the length of the cavity, demonstrated in 3.6. The resonances of the cavity were broadened by the addition of the addition of the $\mathrm{BC}$ waveguides and input waveguide when the resonance profile was evaluated, compared to Figure 2.5. This broadening effect was more pronounced at low 
cavity sizes, and led to a reduction in sensitivity in resonance performance, allowing for greater tolerance for error in commissioning of the cavity. However, to maximise power delivery to the ironsand in the cavity, an electromagnetic field mode which has a large central field density needed to be used. This was evaluated with a unitless 'figure of merit' defined as $\zeta(R, L)$ for each combination of cavity sizes from the same data as Figure 3.4, via

$$
\zeta(R, L)=\frac{U_{E M}(r=0, l=L / 2)}{U_{E M}(R, L)}
$$

with $U_{E M}(R, L)$ from (Eq. 2.4.8). This function evaluates the relative size of the electromagnetic field at the centre of the cavity $U_{E M}(r=0, l=L / 2)$ with respect to the average electromagnetic field density in the cavity $U_{E M}(R, L)$. The results for this evaluation are shown in Figures 3.5a and 3.5b.

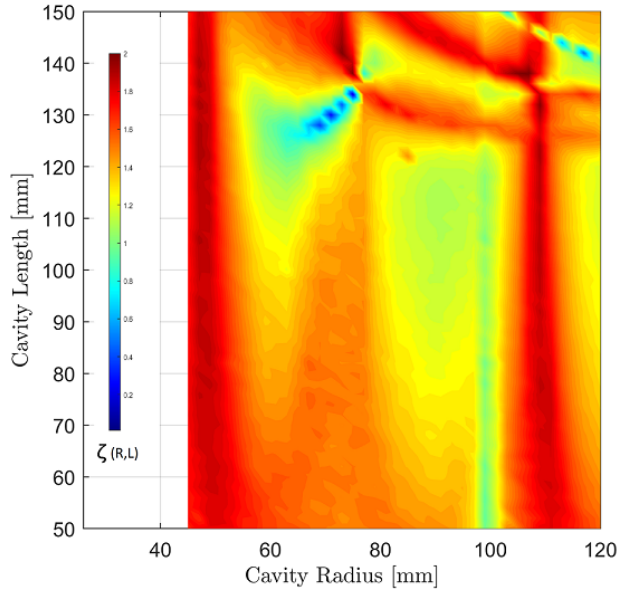

(a) Horizontal; $\zeta(R, L)$ evaluation

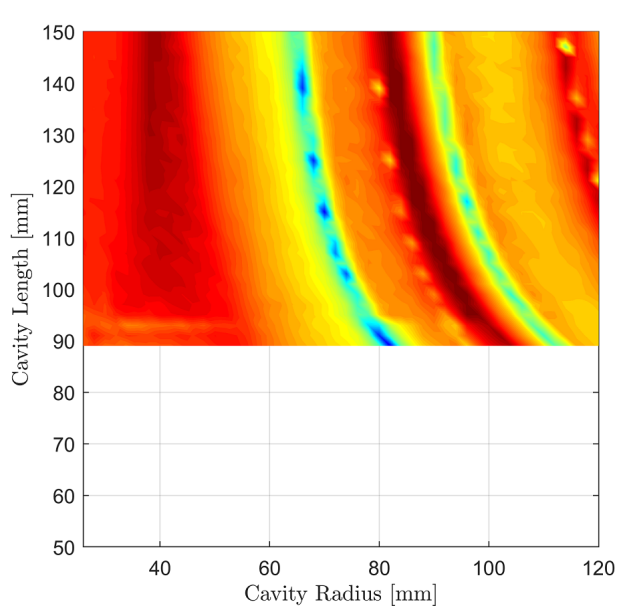

(b) Vertical; $\zeta(R, L)$ evaluation

Figure 3.5: Same parameter sweep as Figure 3.4, however of the average resonance quality, the centralised resonance quality factor $\zeta(R, L)$ from (Eq. 3.4.1) is visualised here.

Figure 3.5a shows a high figure of merit $\zeta$ for radii near $R=50 \mathrm{~mm}$. The characteristic resonant mode of the cavity when $R=47 \mathrm{~mm}$ is the $\mathrm{TM}_{010}$ mode, when (Eq. 2.2.6) is calculated with $\chi_{01}=2.4048$. Due to the resonance broadening from the input waveguide and $\mathrm{BC}$ waveguides, a $R=50 \mathrm{~mm}$ cavity will still result in good resonance, as seen in Figure 3.4a. The cavity length was chosen early on in this project to be $100 \mathrm{~mm}$. If the cavity length is lowered below $100 \mathrm{~mm}$, then the magnitude of the central peak is reduced and the resonance performance was worsened. This could be observed by a reduced 
average field density in Figure 3.4a, and a reduced $\zeta(R, L)$ from Figure 3.5a. If the cavity length is increased to beyond $100 \mathrm{~mm}$, it increases the sensitivity of the performance of the cavity, as another TM mode (observable in Figure 3.4a, specifically the lowest curved mode) begins to compete with the dominant resonant field profile of the $\mathrm{TM}_{010}$ mode.

Thus, the resonant cavity dimensions were determined for commissioning. The dimensions of the cavity are; $R=50 \mathrm{~mm}, L=100 \mathrm{~mm}$ and the input WR340 waveguide orientation is horizontal with respect to the axis of the cavity. It was then useful to visualise the profile of the electric and magnetic fields, to gain an intuition about their profiles within the cavity.

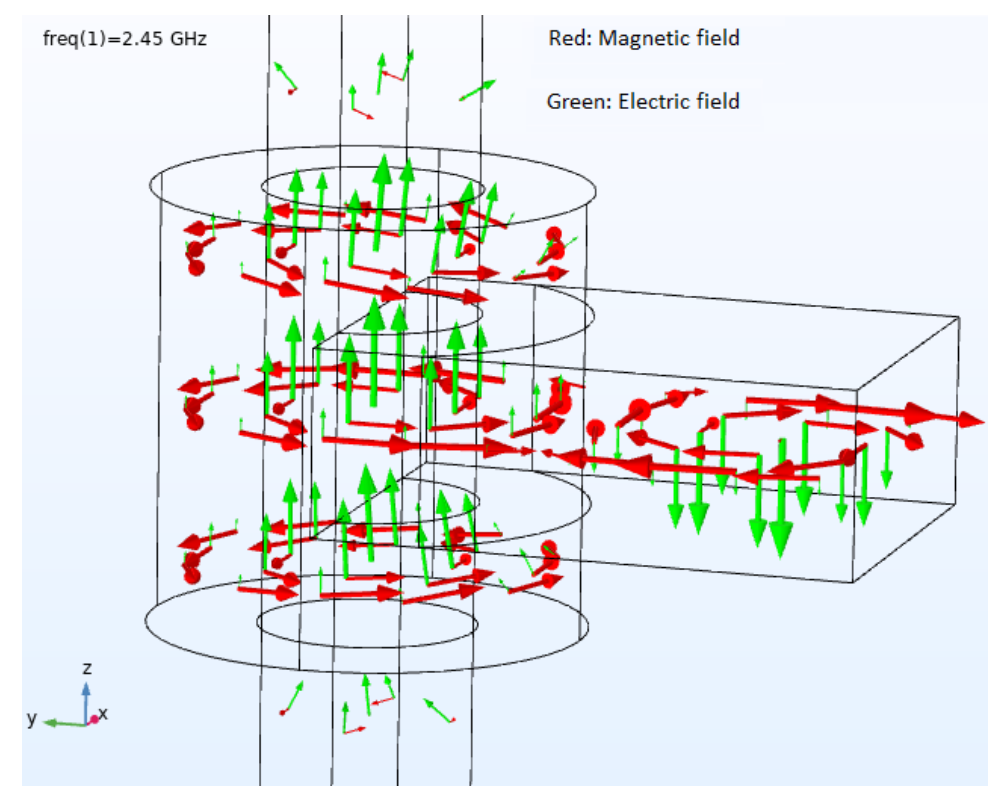

Figure 3.6: Arrow volume plot of the resulting resonance in the cavity with $R=50 \mathrm{~mm}$ and $L=100 \mathrm{~mm}$. The WR340 input waveguide has characteristic $\mathrm{TE}_{10}$ mode propagation (i.e. the circulating magnetic field arrows). No thermal insulation was included in this model. The length and direction of the arrows represent the value of the electric and magnetic fields.

Figure 3.6 demonstrates the $\mathrm{BC}$ effect above and below the cavity, where the amplitude of the fields exponentially decay. The cavity is optimised for electric losses as the longitudinal electric field is centred along the central axis of the cavity. The magnetic field circulates along the perimeter of the cavity.

It should however be noted that this field profile in Figure 3.6 is a static image, but in reality oscillates at $2.45 \mathrm{GHz}$. In addition, the microwaves propagate along the WR340 waveguide at about $0.7 c$ [38]. Thus, there exists a 
continual flux of microwave energy throughout the cavity. In addition, since all types of waves tend to diffract around sharp edges, this explains the 'tilt' of the magnetic field (red) arrows in Figure 3.6 above and below the input waveguide. The average electromagnetic energy density in the cavity in the field profile in Figure 3.6 is $4134 \times 10^{-10} \mathrm{~J} / \mathrm{cm}^{3}$, evaluated with (Eqs. 2.1.5, 2.4.7, 2.4.8).

\subsection{Thermal insulation}

For the resonant cavity to function as a microwave furnace, thermal insulation must be added to the cavity to reduce the radiative heat losses to the surroundings. A large thermal gradient can be maintained by the inclusion of such thermal insulation, increasing $T_{0}$ in (Eq. 2.3.1). Introducing thermal insulation and other media will however affect the resonance performance of the cavity. The resonant frequency of some general (rectangular or cylindrical) cavity packed with a dielectric with permittivity $\varepsilon_{r}$ is reduced by $f \rightarrow f^{\prime} / \sqrt{\varepsilon_{r}}[36]$ (therefore the resonant cavity dimensions also change). The dielectric also must be a low-loss ${ }^{2}$ thermal insulator with low thermal conductivity. AL-25/1700 fibreboard insulation was ideal to use, as it has a low real and imaginary permittivity $\varepsilon_{r}$ and thermal conductivity $k_{t}$. In further work, a large BN tube with outer radius $R=20 \mathrm{~mm}$ would need be placed through the cavity to pass the ironsand through. This required the 'cutting' of the alumina fibreboards into a set of annuli with inner radii of $22.5 \mathrm{~mm}$ and outer radii of $50 \mathrm{~mm}$, to be fitted into the cavity. The resulting annuli are shown in Figure 2.8.

To evaluate the effect of adding thermal insulation, the density of the electromagnetic field $U_{E M}$ from (Eq. 2.1.5) is evaluated along the central axis of the cavity, from one end of one $\mathrm{BC}$ waveguide to the end of the other ${ }^{3}$, and is shown in Figure 3.7.

The average total electromagnetic energy density $U_{E M}$ in the cavity is reduced by a factor of $1 / 2$ from $4134 \times 10^{-10}$ to $2125 \times 10^{-10} \mathrm{~J} / \mathrm{cm}^{3}$. Some of the electromagnetic field energy has leaked further into the $\mathrm{BC}$ waveguide. The microwave power leakage from the $\mathrm{BC}$ waveguides increased by a factor of 10 to $2.49 \times 10^{-7} \mathrm{~mW} / \mathrm{mm}^{2}$. The total leaked microwave power is of the order of microwatts.

\footnotetext{
${ }^{2}$ For the thermal insulation to be considered a low-loss material, the imaginary permittivity must be low, i.e. $\varepsilon_{r}^{\prime \prime} / \varepsilon_{r}^{\prime}<0.01$.

${ }^{3}$ For example from the top to the bottom of Figure 3.3.
} 


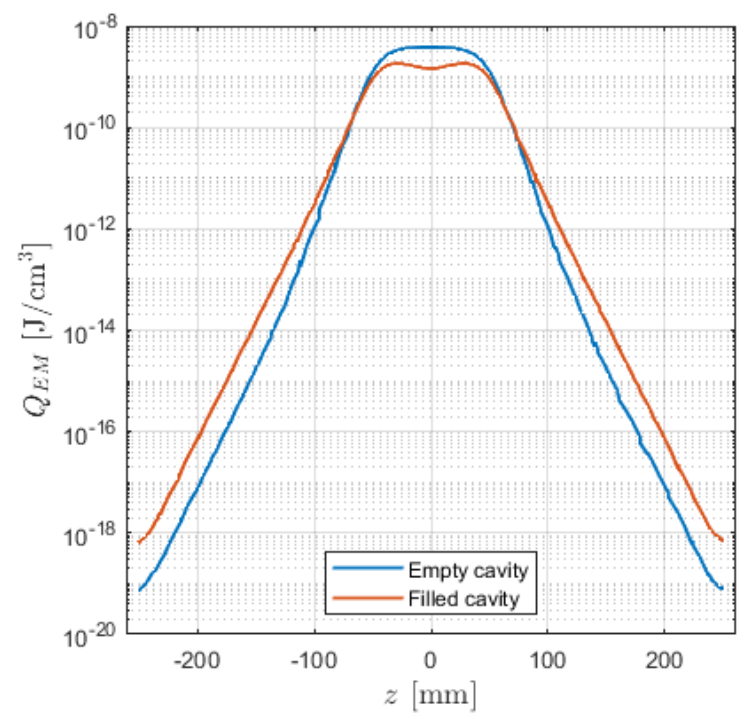

Figure 3.7: The inclusion of thermal insulation reduced the axial electromagnetic field energy density (blue), and appeared to produce a 'bi-modal' type of field energy density profile along the central axis of the cavity (orange).

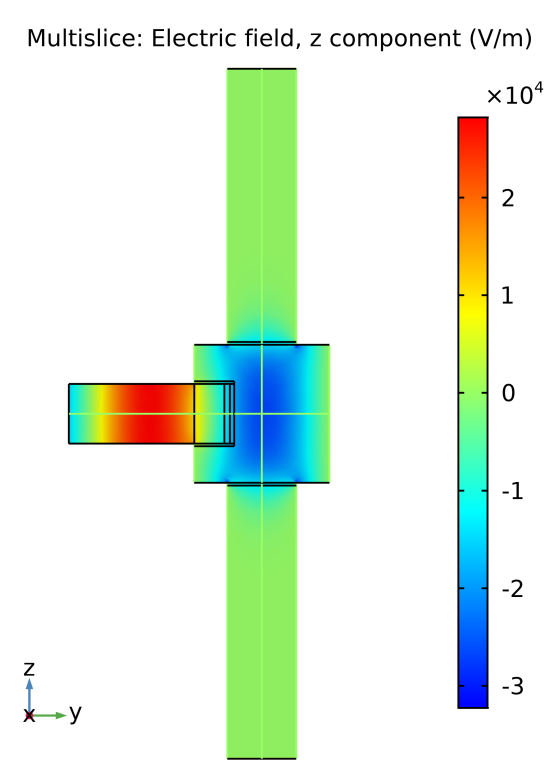

(a) $E_{z}$ before insulation inclusion.

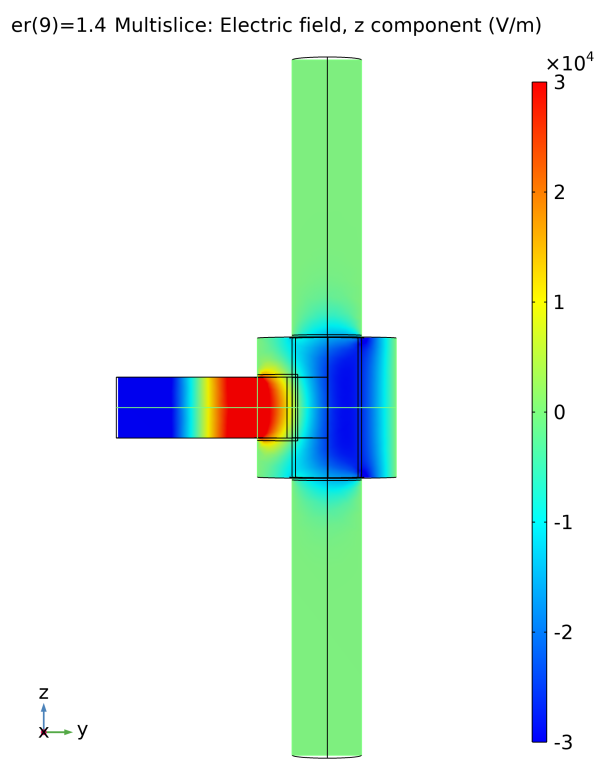

(b) $E_{z}$ after insulation inclusion.

Figure 3.8: Cross-section of prototype furnace simulation $(R=50 \mathrm{~mm}, L=$ $100 \mathrm{~mm}$ ) showing $E_{z}$ field intensity for cavity with (3.8b) and without (3.8a) AL-25/1700 Alumina fibreboard annuli (inner radius $22.5 \mathrm{~mm}$ ). No BN tubes were included in this model. 
Comparing Figure 3.8b to Figure 3.8a demonstrates that the electromagnetic field mode is slightly displaced from the centre of the cavity (note the colouring in Figures 3.8a and 3.8b). The peak electromagnetic field power is still predominantly on the central axis of the cavity. The core functionality of the cavity is unchanged, and is then fit to function as a furnace if ironsand is placed into the centre of the cavity. The BN which contains the ironsand has a real permittivity of approximately 4.2. However, the volume of the BN tube with respect to the size of the cavity is negligible, so only barely affects resonance performance of the cavity beyond the perturbation added by the insulation itself.

\subsection{Optical access}

Measuring temperatures in a microwave furnace presents its own problems, primarily that no thermocouples can be placed within the cavity without affecting the heating dynamics of the cavity. To measure temperature within the furnace, having direct optical contact to the centre of the cavity is essential. For ease of physical measurement, the optical access holes are placed $90^{\circ}$ around the microwave cavity from the input waveguide. These optical access holes need to have a minimum diameter $h$, such that the aperture $M$ of the pyrometer has direct optical contact to the measurement spot $s$ with distance $L$ between the two. The measured object is the side of the BN tube holding the ironsand, with radius $u$. The radius of the cavity is $R$, and the wall thickness is $d$. A table to keep track of the relevant dimensions is then useful.

\begin{tabular}{||c|c||}
\hline Optical access fixed dimensions & Variable \\
\hline \hline Cavity radius & $R=50 \mathrm{~mm}$ \\
\hline Cavity wall width & $d=2 \mathrm{~mm}$ \\
\hline Spot size & $s=0.5 \mathrm{~mm}$ \\
\hline Aperture size & $M=12 \mathrm{~mm}$ \\
\hline Focal distance & $L=150 \mathrm{~mm}$ \\
\hline BN tube radius & $u=7.5 \mathrm{~mm}$ \\
\hline
\end{tabular}

Table 3.2: The table of relevant fixed dimensions used for the optical access hole diameter calculation. 
This, however, may leak significant microwave radiation if the hole size is too large. To further complicate the calculation, the curvature of the cavity affects the effective beyond cutoff length of the hole. This can be observed if a top-down view is used for the wall.

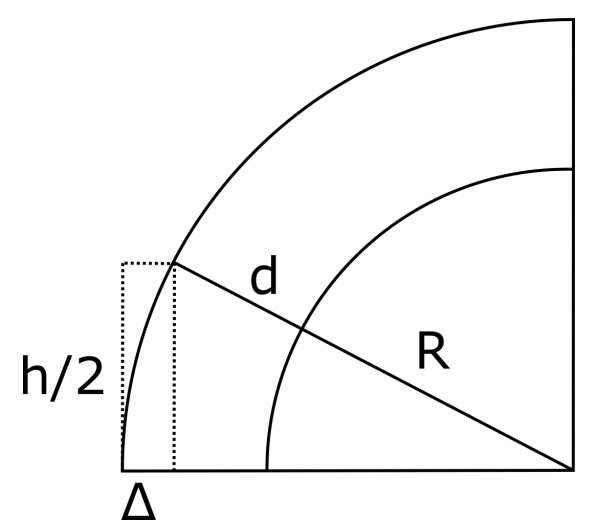

Figure 3.9: Exaggerated diagram of geometrical correction to wall depth. View is from above cavity. This diagram exemplifies the reduced effective depth of the wall due to the curvature of the cavity. A correction factor $\Delta=R+d-\sqrt{(R+d)^{2}-(h / 2)^{2}}$ was then calculated.

By using the correction factor from Figure 3.9, the new wall width was calculated via Pythagoras' Theorem:

$$
\begin{aligned}
& d^{\prime}=d-\Delta \\
& d^{\prime}=\sqrt{(R+d)^{2}-\left(\frac{h}{2}\right)^{2}}-R
\end{aligned}
$$

The total leaked microwave power in the context of this geometry could be calculated via

$$
\mathrm{dB}=-\frac{10}{\ln (10)} \frac{2}{c}\left(\sqrt{(R+d)^{2}-\left(\frac{h}{2}\right)^{2}}-R\right) \sqrt{\left(\frac{4 \pi c \chi_{11}^{\prime}}{h}\right)-\omega_{0}^{2}}
$$

which is adapted from (Eq. 3.3.1). If a hole height of $h=10 \mathrm{~mm}$ and a driving frequency of $\omega_{0}=1.54 \times 10^{10} \mathrm{rad} / \mathrm{s}$ is used, the resulting decibel attenuation is $35.3 \mathrm{~dB}$. To reduce the leaked microwave power, additional beyond cutoff tubing is required. This can be visualised in an exaggerated diagram in Figure 3.10. 


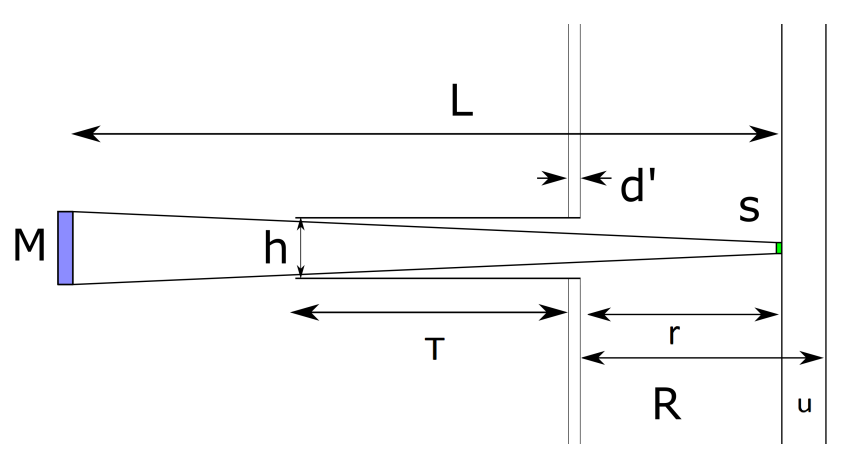

Figure 3.10: Exaggerated diagram of optical access limitation by pyrometer. View is from the side of cavity. A description of initial parameters in this plot is given in Table 3.2, and the rest given in Table 3.3.

\begin{tabular}{||c|c||}
\hline Parameter & Variable \\
\hline \hline Optical access hole width & $h$ \\
\hline Optical access tubing length & $T$ \\
\hline Corrected cavity wall width & $d^{\prime}$ \\
\hline Cavity wall width correction term & $\Delta$ \\
\hline Distance from cavity to BN tube & $r$ \\
\hline
\end{tabular}

Table 3.3: The table of relevant parameters used specifically for the optical access hole calculation.

If tubing with length $T$ is included, the minimum optical access diameter $h$ increases. Using similar triangles, a relationship between the optical access tube dimensions can be found.

$$
\frac{M-s}{L}=\frac{h-s}{r+d^{\prime}+T}
$$

Knowing $d^{\prime}$ from (Eq. 3.6.1), a less than elegant exact solution for $h$ can be found.

$$
h=\frac{-\nu \eta}{\left(\nu^{2}+\frac{1}{4}\right)}+\frac{\sqrt{(r+d)^{2}\left(4 \nu^{2}+1\right)-\eta^{2}}}{2\left(\nu^{2}+\frac{1}{4}\right)}
$$

with $\nu=\frac{L}{M-s}$ and $\eta=\nu s-T-r$ such that $h$ from (Eq. 3.6.4) is the minimum tubing diameter for any tubing length $T$. Returning to (Eq. 3.6.2): the required tubing length $T$ to attenuate the microwave intensity by some 
fixed $\mathrm{dB}$, with optical access tube diameter $h$ is written $\mathrm{as}^{4}$ :

$$
T=R-\sqrt{(R+d)^{2}-\left(\frac{h}{2}\right)^{2}}+\frac{c \ln (10)}{20 \sqrt{\left(\frac{2 \pi c \chi_{11}^{\prime}}{h}\right)^{2}-\omega_{0}^{2}}} \mathrm{~dB}
$$

where a positive attenuation in $\mathrm{dB}$ is input in the above expression. The expressions in (Eq. 3.6.4) and (Eq. 3.6.5) are then plotted in Figure 3.11 for a range of decibel attenuation values.

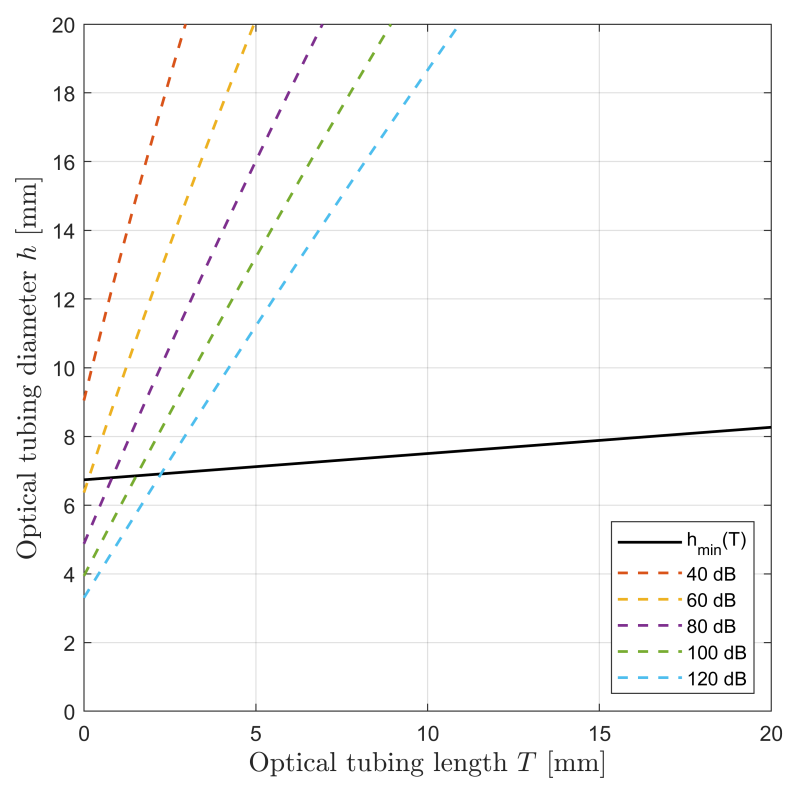

Figure 3.11: Diagram of optical access tube geometries resulting in a range of $2.45 \mathrm{GHz}$ microwave attenuation. The black line describes the minimum optical tube diameter, and the dashed coloured lines describe the amount of $2.45 \mathrm{GHz}$ microwave attenuation for each combination of tube dimensions.

The designed tube must have a diameter above the black line in Figure 3.11 , so that the pyrometer used in this work has direct optical access to the measurement spot. The length of the tube should ideally be as long as possible to minimise microwave leakage, but not too long as to block the optical access. Tubing length of $5 \mathrm{~mm}$ with a conveniently procurable inner tube diameter of $10 \mathrm{~mm}$ would suffice to attenuate the microwave radiation by over $120 \mathrm{~dB}$. However to 'over-engineer' the safety of the system, an optical tubing length of

\footnotetext{
${ }^{4}$ Unfortunately, there is no closed form expression for $h(T)$ in (Eq. 3.6.5), so $T(h)$ is derived and the inverse of that function plotted in Figure 3.11.
} 
$20 \mathrm{~mm}$ was used for this work. This maximised the optical safety of the tube, while maintaining direct optical contact.

Optical access tubes with inner diameter $h=10 \mathrm{~mm}$ and length $T=20 \mathrm{~mm}$ were manufactured, then onto the side of the cavity after a hole was drilled out. Optical access tubes are incorporated into the computational model, and a full figure of the completed model is included in Chapter 5. This concludes the initial design work to confidently commission the microwave cavity so such that the cavity is safe to operate, and useful for laboratory testing, and therefore suitable for experimental verification and refinement of computational results. Table 3.4 is the collection of final cavity dimensions used to build the cavity.

\begin{tabular}{||c|c||}
\hline Dimension & Value \\
\hline \hline Cavity radius & $50 \mathrm{~mm}$ \\
\hline Cavity length & $100 \mathrm{~mm}$ \\
\hline BC waveguide radius & $25 \mathrm{~mm}$ \\
\hline BC waveguide length & $200 \mathrm{~mm}$ \\
\hline Optical access radius & $5 \mathrm{~mm}$ \\
\hline Optical access length & $20 \mathrm{~mm}$ \\
\hline WR340 input waveguide orientation & Horizontal \\
\hline
\end{tabular}

Table 3.4: Table of commissioned dimensions to build the microwave cavity. The radii written in this table refer to the inner radius of that part. The width of the copper tubing used to build the cavity was $2 \mathrm{~mm}$. 


\section{Chapter 4}

\section{Experimental methods}

Having finalised the design of a laboratory-scale microwave furnace in the previous chapter, the next stage of this research was to build and test that prototype. In this chapter, the experimental components used in this work are discussed, after which the various measurement equipment is introduced. For many pieces of equipment, calibration and analysis of instrumental errors was required. This chapter concludes with pictures of the experimental setup and a definitive simple experimental method to refine the computational model with. Some explorational experimental results are also included as they informed what various artefacts could be produced and observed during testing.

\subsection{Transmission line components}

To transfer microwave power from a magnetron to a resonant cavity, several transmission line components are necessary. The components within the waveguide system which injects microwave power into the cavity, collectively form the transmission line. Each component is based on the standard WR340 width of $86.36 \mathrm{~mm}$ and a height of $43.18 \mathrm{~mm}$, allowing the system to be easily taken apart and put together again. In this section, each transmission line component is discussed separately. The standard order of components [81] in the transmission line is employed, and a picture of this setup is shown as Figure 4.1.

Two pictures of each component are included at the end of the relevant sub-sections; one of the external body of the component, and another to observe the internal structure of that component. 


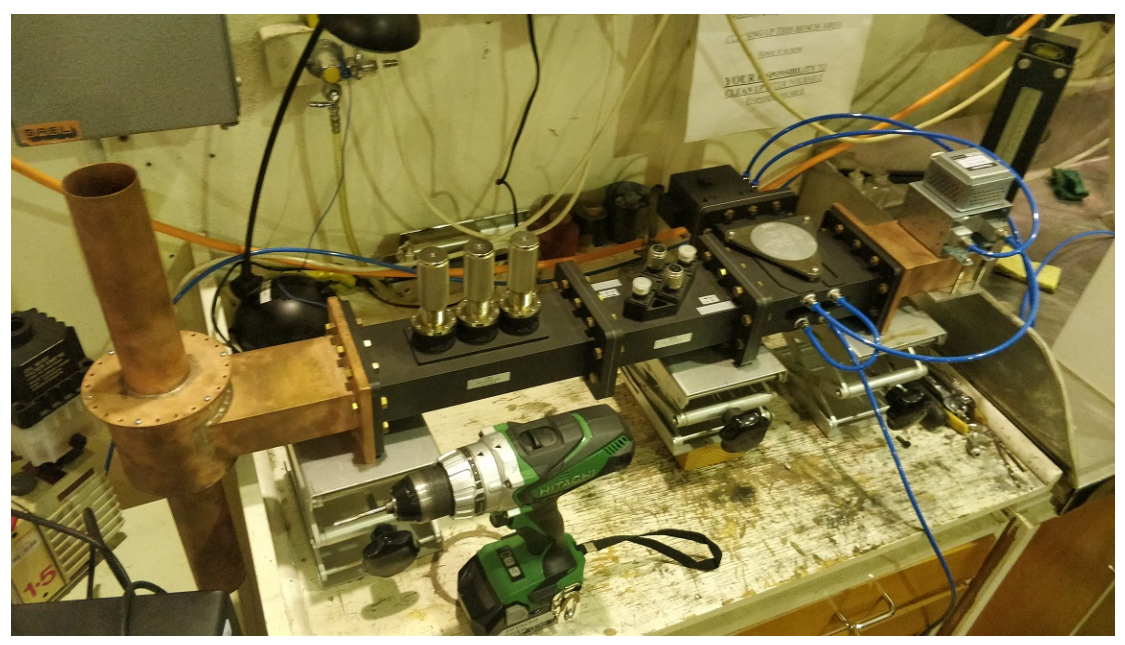

Figure 4.1: Basic setup of RF transmission line. From right to left; magnetron launch, circulator, terminator (behind circulator), dual-directional coupler, three-stub tuner and microwave furnace. All components are discussed, and assembled as in this picture of the setup. The system is raised from the bench by lab jacks. Water cooling tubing (blue tubes on the right) for the magnetron, terminator and circulator is included. Note that the optical access tubes had not yet been added to the side of the microwave cavity.

\subsubsection{Magnetron launch}

To produce microwave power, mains voltage $(230 \mathrm{~V}, 50 \mathrm{~Hz})$ is converted to a high DC voltage of $4.2 \mathrm{kV}$ by a custom-built potentiometer-controlled Enbiens YB-MP1000 power supply. This high voltage is fed to a Panasonic $1 \mathrm{~kW}$ 2M261-M32 magnetron. The magnetron uses that high DC voltage to produce $2.45 \pm 0.05 \mathrm{GHz}$ power. The typical efficiency of a $1 \mathrm{~kW}$ magnetron is $70 \%$ [82].

The method by which a magnetron functions is by thermionic emission of electrons from a hot central cathode [82] shown in Figure 4.2. There are high strength magnets, with magnetic field lines parallel to the central axis of the resonant cavity of the magnetron, which can be seen as the black cylinders in the magnetron in Figure 4.3a. The emitted electrons have their paths deflected by the magnetic field in the interaction chamber, between the heated cathode and anode block. This leads the electrons to pass over a number of small slits in the anode block, through which a capacitive build up of charge occurs on each side of the slit. Beyond the slit is a cylindrical cavity through which the diffusing movement of charge due to that aforementioned capacitive build up 


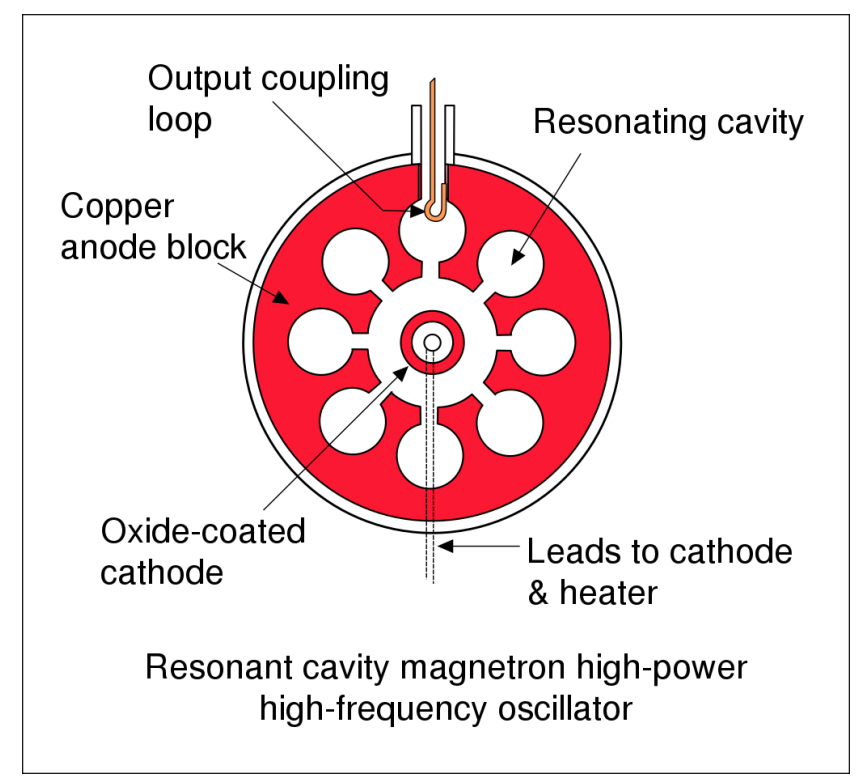

Figure 4.2: Cross-sectional diagram of typical magnetron structure. The magnetic field lines are parallel with the axis of the structure (perpendicular to the page) [83].

produces a changing magnetic field within the cavity. The coupled dimensions of the slit and the cavity result in resonance at $2.45 \mathrm{GHz}^{1}$. In a magnetron of this type, there are multiple small cavities for this resonance to occur. These cavities are then coupled to a coaxial coupling line which guides the microwave power from the cavity to the WR340 waveguide.

The ceramic probe from which the microwave power is emitted is seen in Figure $4.3 \mathrm{~b}$, and is off-set by $\lambda / 4^{2}$ from the closed-side of the magnetron waveguide component. This setup launches microwave power into the transmission line. The magnetron used in this work is a water-cooled upgraded version of the standard Panasonic 2M261-M32 magnetron (that is a commercial component for domestic kitchen microwave ovens). Water-cooling improves magnetron performance over long active periods.

\footnotetext{
${ }^{1}$ The exact frequency may drift from $2.45 \mathrm{GHz}$ due to a varying load, for example by about $\pm 50 \mathrm{MHz}$ in a standard microwave oven magnetron.

${ }^{2}$ The wavelength of $2.45 \mathrm{GHz}$ microwaves is $\lambda=12.26 \mathrm{~cm}$
} 


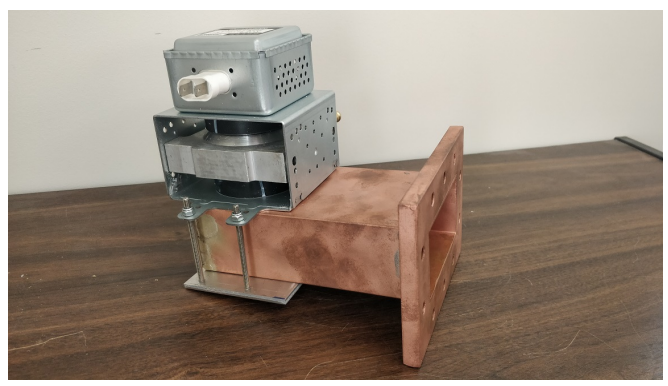

(a) Magnetron launch body

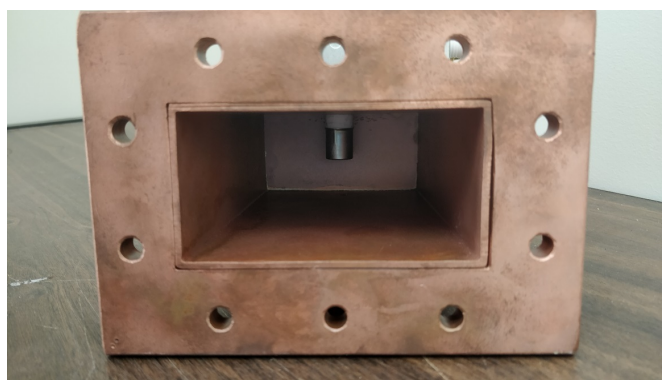

(b) Magnetron internal view

Figure 4.3: The magnetron launch has three sections. The first of which is a heating block (top). The second is cathode-anode 2M261-M32 magnetron (center). The third is a water cooling heat-sink (around the magnetron block), where the microwave power is produced. The final part is ceramic probe, which protrudes into the transmission line (Figure 4.3b).

\subsubsection{Circulator}

Allowing reflected microwave power to propagate back towards the magnetron would result in heating the magnets in the magnetron, thus reducing the magnetic field produced by those magnets. This in turn affects the propagation of emitted electrons from the cathode, and reduces the output microwave power of the magnetron.

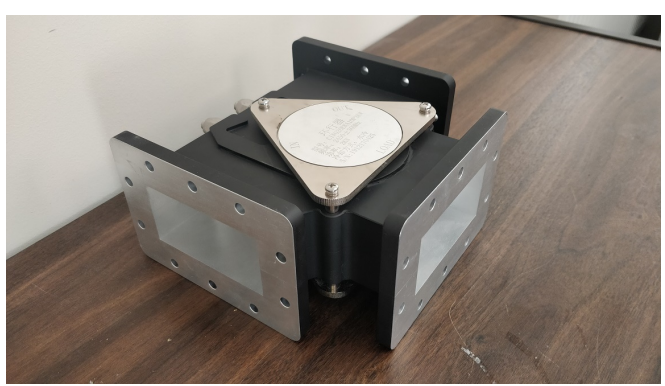

(a) Circulator body

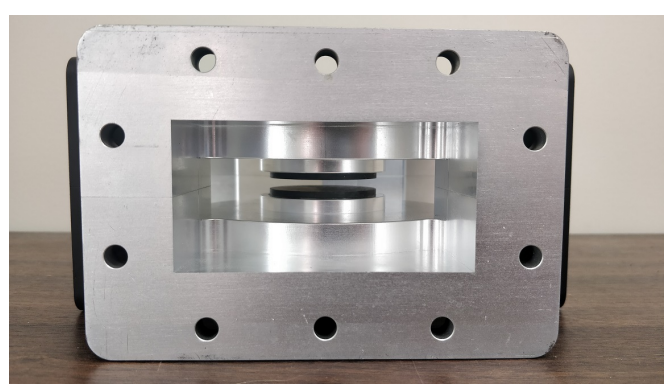

(b) Circulator internal view

Figure 4.4: The two black disks are the ferrite spinel magnets, which allow for selected directional propagation of electromagnetic radiation through the component; i.e. from IN to OUT, or from OUT to LOAD.

To avoid these problems, reflected power is blocked from reaching the magnetron, by using a circulator shown in Figure 4.4a. The circulator directs reflected microwave power from the cavity into the terminator (discussed next). 
The directional control is performed by the circulator which contains two strong spinel ferrite magnets. These magnets allow electromagnetic fields to pass through the magnetic fields generated by the spinel ferrite magnets in only one direction with the ferromagnetic Faraday effect [84]. This is due to the inherent spin direction of the electrons within the ferrite crystal structure [85]. Microwave radiation directed from the magnetron launch (IN) to the microwave cavity (OUT) passes straight through the circulator, whilst the majority of the radiation propagating from the microwave cavity (OUT) to the terminator (LOAD) is reflected into a 90 degree arm of the waveguide ${ }^{3}$. In this way, reflected power to the magnetron is attenuated by $30 \mathrm{~dB}$ by the circulator used in this work, therefore preventing magnet overheating and therefore mitigating damage done to the magnetron.

\subsubsection{Terminator}

Reflected microwave power is directed to the terminator via the circulator. The purpose of the terminator is to maintain high microwave power output of the magnetron. The internal body of the terminator can be observed in Figure $4.5 \mathrm{~b}$ and is comprised of three tuned stubs and two water tubes. This results in continuous deposition of reflected microwave power from the cavity into the water. The water is heated by the deposited microwave power. This heating process can be used to calibrate power transfer dynamics within the transmission line. The temperature difference is monitored with customdeveloped LabVIEW software with a K-type thermocouple wrapped in PTFE ( Polytetrafluoroethylene) sealing tape to thermally isolate the thermocouple from the from air.

\subsubsection{Three-stub tuner}

The three-stub tuner allows for control of the directional propagation of the microwave radiation within the transmission line. Using the three-stub tuner, the combined reflected power from the three-stub tuner and microwave furnace cavity can be minimised, therefore optimising the microwave power deposition rate in the microwave cavity. Each tuning knob on the external body is marked

\footnotetext{
${ }^{3}$ Or from the terminator (LOAD) to the magnetron source (IN). Ideally however, this does not happen as all reflected power should be deposited in the terminator.
} 


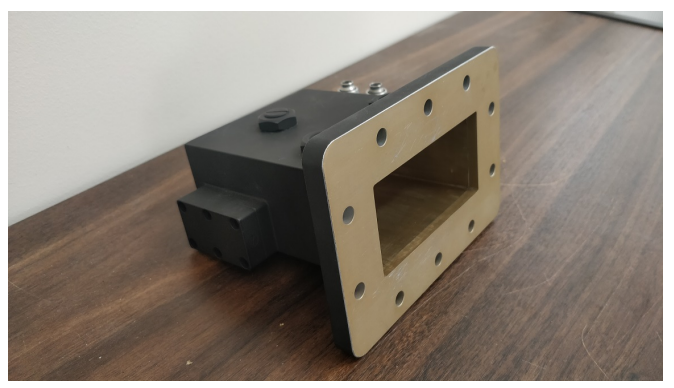

(a) Terminator body

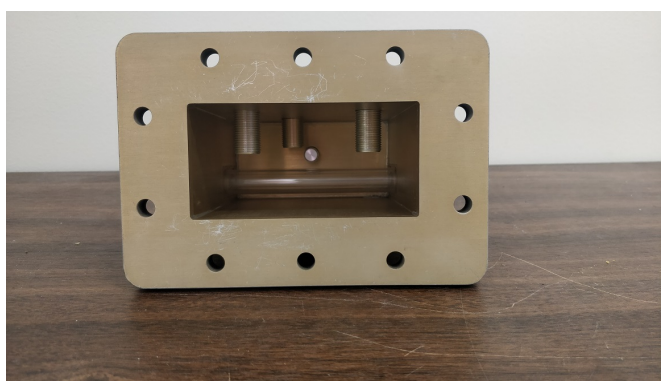

(b) Terminator internal view

Figure 4.5: The three stubs and water tubes in the terminator are designed as a load for microwave power. This component was also used for microwave power magnitude calibration.

at four equally spaced points around the base of the tuner, the zero point of the tuner corresponds to full retraction of the stub within the three-stub tuner. A complete $360^{\circ}$ rotation of a stub corresponds to a protrusion of $1.21 \pm 0.02 \mathrm{~mm}$. The maximum number of stub turns is approximately 21 turns, which corresponds to a protrusion depth of $25.4 \mathrm{~mm}$. Each stub has an outer diameter of $15.66 \pm 0.02 \mathrm{~mm}$, and has a spherically-shaped end to prevent arcing within the waveguide. The distance between the stubs is $45.68 \pm 0.02 \mathrm{~mm}$. By rotating the screws, the stubs are protruded into, and retracted from the waveguide.

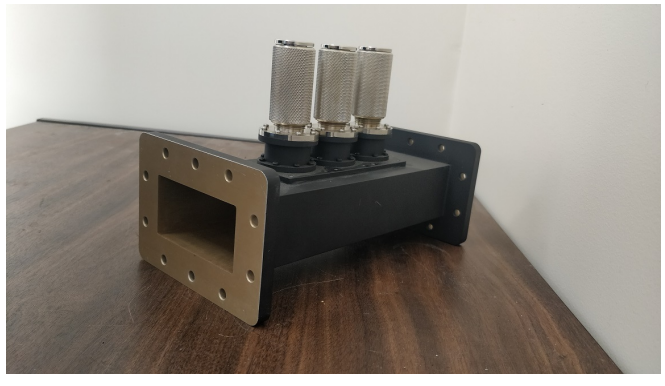

(a) Three-stub tuner body

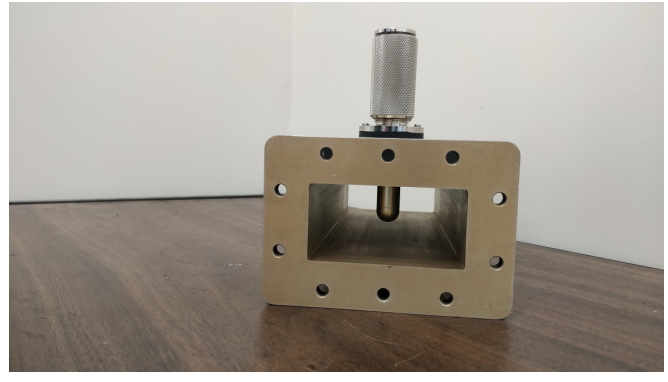

(b) Three-stub tuner internal view

Figure 4.6: The three stubs with spherical end caps can be retracted and protruded by turning the tuners from the outside of the component. This changes the reflection and transmission properties of incident microwave power through this component, allowing for optimised microwave heating of ironsand in the cavity.

Some RF power from the magnetron is reflected by this component. The rest 
of the RF power that is not reflected, is incident to the microwave cavity. Then, some of that resulting transmitted power is then reflected by the microwave cavity. The RF power reflected by the microwave cavity is then again incident to the three-stub tuner. This results in further transmission and reflection of $\mathrm{RF}$ power, this time the source of the power being the microwave cavity. The amplitude and phase of those waves propagating towards the magnetron are tune-able (by the three-stub tuner) to destructively interfere with one-another to minimise power transmitted towards the terminator/magnetron. A standing wave between the microwave cavity and three-stub tuner is produced as a result. This maximises the power transfer from the magnetron to the microwave cavity. However, this occurs only when the tuning is 'ideal', thus it remains essential to have a circulator, and terminator in the system of RF electronics. In addition, the reflection dynamics of the system change significantly during heating of the ironsand in the furnace.

\subsubsection{Dual-directional coupler}

Since the directional power can be modified by the protrusion depths of the three-stub tuner, the amplitude of power travelling in each direction along the transmission line is of interest. The dual-directional coupler is used to detect the directional RF power by coupling the WR340 waveguide to two coaxial transmission lines. The coupling is done via two identical $23 \mathrm{~mm}$ holes out of the centre of the top of the dual-directional coupler. On the other side of each hole is a coaxial line, angled at $45^{\circ}$ from the plane of the transmission line, clearly seen in Figure 4.7a. The coaxial line has a ratio of outer-to-toinner-conductor radius of 2.3 , resulting in wave impedance of $50 \Omega$. There are two coaxial lines, and the RF power is reverse-coupled into these lines due to the geometrical design of the holes [86]. The microwaves then propagate along the connected coaxial line in the opposite direction from which they were propagating in the transmission line. The hole sizes result in $60 \pm 1 \mathrm{~dB}$ reverse coupling. A RF detector antenna is connected at the end of each coaxial lined. This detector reads the propagating RF power through the main transmission line. At the other end of each coaxial line, a $50 \Omega$ terminator is connected. This terminator prevents signal reflection and gives high directivity $(>30 \mathrm{~dB})$ to each directional power measurement.

The advantage of using the setup order as in Figure 4.1 is that it allows 


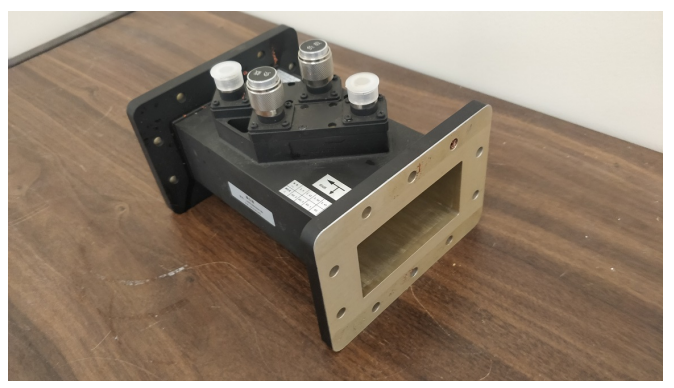

(a) Dual-directional coupler body

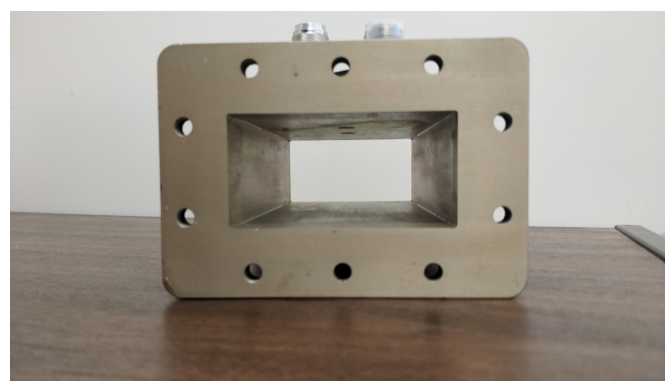

(b) Dual-directional coupler internal view

Figure 4.7: The dark knobs in Figure 4.7a are $50 \Omega$ terminators. The other ends (pictured here with white plastic covers) are connected to AD8317 RF-DC detectors via coaxial cables for directional power analysis. Further details on the connection of these detectors is included in Section 4.3.2.

for continuous high power output of the magnetron, control over microwave propagation within the system with the three-stub tuner, and analysis of that directional power propagation throughout the system during heating with the dual directional coupler.

\subsubsection{Microwave cavity}

The microwave cavity was then manufactured in-house by Lead R\&D Engineer John Futter at GNS Science, with the design parameters shown in Table 3.4. The modelled prototype microwave cavity model had best performance with radius $R=46 \mathrm{~mm}$ (shown in Figure 3.4a, but the closest procurable copper tubing had a radius of $50 \mathrm{~mm}$. Due to the 'smearing' of electromagnetic modes in the same figure, this radius difference was acceptable and would still result in good microwave resonance within the microwave cavity. The pieces of copper tubing (microwave cavity body and $\mathrm{BC}$ waveguides) were then brazed and soldered together as modelled in Figure 3.3.

The radius of the microwave cavity was $50 \mathrm{~mm}$, the length was $100 \mathrm{~mm}$, the WR340 waveguide had width $86.36 \mathrm{~mm}$, height $43.18 \mathrm{~mm}$. The BC waveguides had radius $25 \mathrm{~mm}$ and length $200 \mathrm{~mm}$. To include Alumina fibreboard AL25/1700 thermal insulation, the top plate of the microwave cavity and BC tube were brazed together as can be seen in Figure 4.8, and are connected to the rest of the microwave cavity via 36 screws. It is noted that the optical access holes were not yet implemented at this stage of furnace development and were 


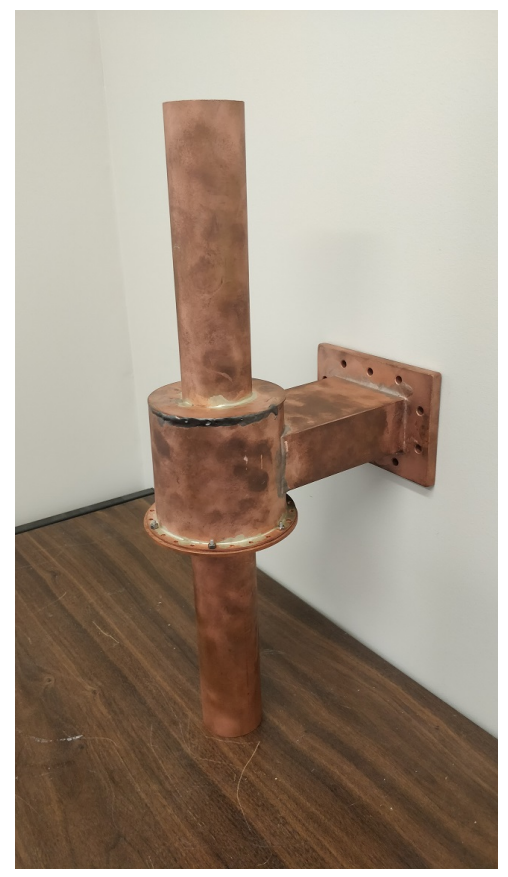

Figure 4.8: First build of the microwave cavity by John Futter. A dozen screws were screwed in for the purpose of the picture. The input WR340 waveguide is on the right, and the dimensions of the microwave cavity and $\mathrm{BC}$ waveguides were verified and were in agreement with the commissioning outline.

added later, once the pyrometers used in this work were chosen.

\subsection{Experimental setup}

Once the furnace cavity had been manufactured as in Figure 4.8, the prototype furnace was assembled by connecting the rest of the transmission line components as per Figure 4.1. At this point, none of the pyrometers, the AD8317 logarithmic microwave power detectors, or the thermal insulation were yet available, nor had the LabVIEW software been developed to process the power measurement data. As various components arrived, the system was upgraded and continually tested. Initial tests were done to check microwave leakage sites on the setup to ensure safe operation. The initial setup was arranged horizontally and placed near a direct water source for water-cooling of various components, as is shown as Figure 4.9. The assembly was placed horizontally for ease of initial testing with various crucibles.

The $0 \rightarrow 6 \mathrm{~V}$ voltage control was approximately linearly related to the 


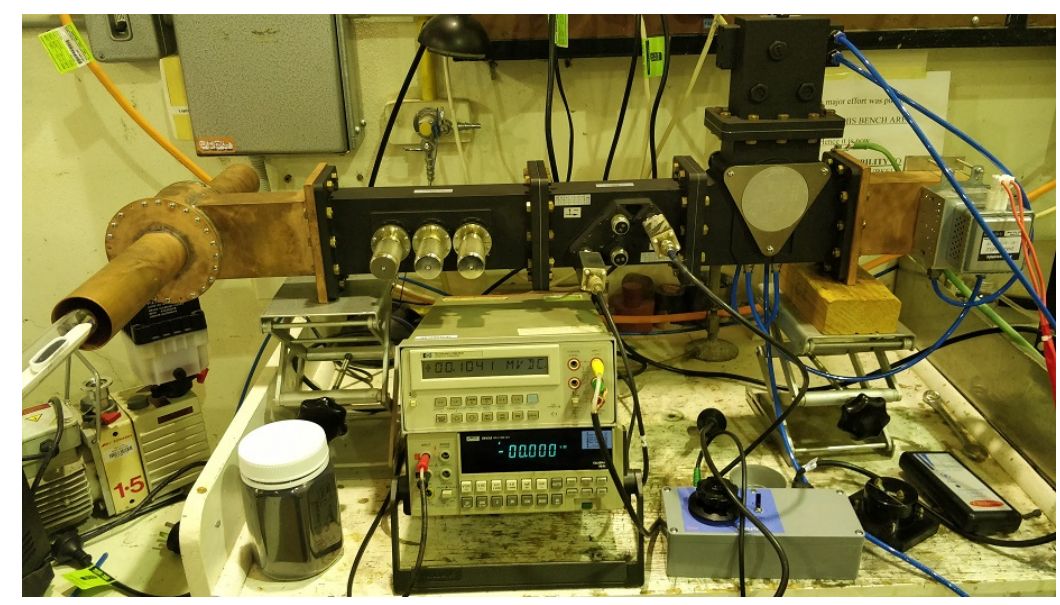

Figure 4.9: Initial assembly of the prototype microwave furnace for preliminary testing. From left to right: alumina boat filled with ironsand, microwave furnace cavity, three-stub tuner, dual-directional coupler, terminator (above), circulator (below), magnetron. Below on the right (with the blue sticker) is the power controller on the right with continuous voltage control from $0 \rightarrow 6 \mathrm{~V}$. Below on the left are two multimeters to qualitatively measure the time-averaged directional microwave power propagating through the transmission line.

output power of the magnetron. During initial commissioning, some power measurement devices were available, but uncalibrated, and were used for purely qualitative measurements. An old Ircon modline two-colour pyrometer was also used for the same reason. Key observations indicated that the reflected power could be reduced by tuning the stubs of the three-stub tuner. Simultaneously, the temperature measured by the two-colour pyrometer reached the maximum measurement temperature $\left(1400^{\circ} \mathrm{C}\right)$. This part of the research was focused on ensuring that the microwaves were indeed able to quickly heat TTM ironsand. Small alumina ceramic crucibles known as 'boats' were centred in the cavity and heated with the microwave furnace. These boats were placed into closed-ended quartz tubes. Quartz is both optically and microwave transparent, which allows for observation of the microwave heating of the TTM ironsand placed into the cavity. Many times during this phase of research, plasma was produced in the cavity by the high microwave power once the three-stub tuner positions were optimised. This resulted in melting of sections of the ironsand and alumina boats, which is discussed further in Chapter 4.4 .

Later, once the pyrometers had arrived, two optical access tubes were brazed onto the side of the microwave cavity, thermal insulation fibreboards 
were packed into the cavity, and the furnace cavity was oriented upright for testing. The LabVIEW software to process the data was developed at this stage. A hole was drilled into the back of the magnetron launch component to ventilate the transmission line with $\mathrm{N}_{2}$ gas during operation, in order to prevent the outside of the BN tube from oxidising at high temperatures [87]. Thin BN rods (15 mm diameter, $300 \mathrm{~mm}$ length) were added and a hole was drilled down the centre of the length $(10 \mathrm{~mm}$ diameter, $100 \mathrm{~mm}$ length) from one end of one of the tubes for ironsand packing and heating in the microwave furnace. Water cooling copper tubing was also brazed to the outside of the base of the $\mathrm{BC}$ waveguide to reduce the temperature of the copper furnace. Kaowool thermal insulation was packed into the top of the BC waveguide, to cover the K-type thermocouple and to reduce convective heat loss, shown in Figure 4.10.

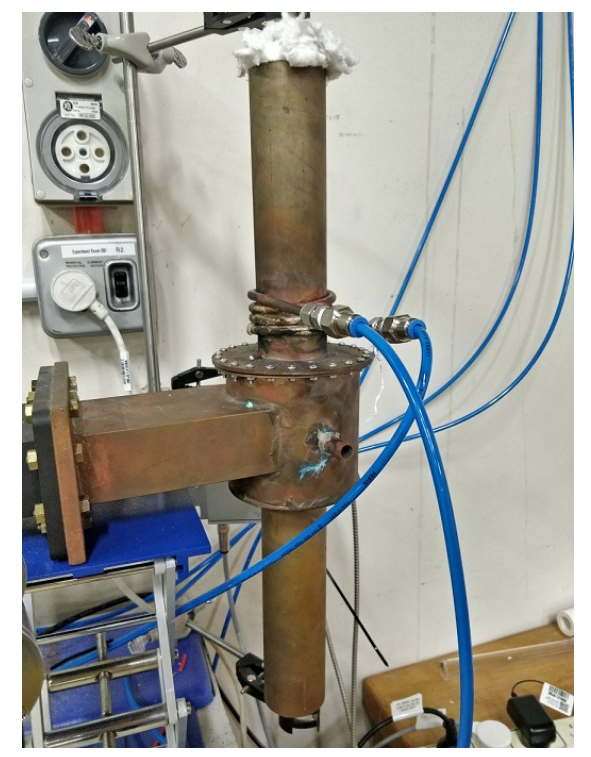

Figure 4.10: Final microwave furnace setup picture. The optical access tubing, the water cooling jacket, kaowool plugging, and BN tubes are included in this picture, in addition to being placed vertically again.

Much data initially extracted was useless due to the 'clipping' of the power measurement, along with the poor data processing practice of pure averaging of signal intensity. This was corrected as a measurement improvement process. After reducing the power of the power measurement signal by adding $20 \mathrm{~dB}$ attenuators, measurements were improved. Then the measurements were further improved by subtracting the $>10 \mathrm{kHz}$ aliased noise by adding a $1 \mu \mathrm{F}$ capacitor 
between the output and ground lines of the RF-DC detectors, described in Section 4.3.2. After this, the voltage output of the RF-DC detectors were calibrated to a power measurement with the water heating calibration. In addition, the water flow rate was measured with an RS Pro Flow Meter, described in more detail in Section 4.15.

\subsection{Measurement processes}

The following sections introduce the measurement equipment and calibration procedures to extract data from the measurements to compare to the simulations in Chapter 5. This section includes RF-DC detectors to measure the power propagation throughout the system. A reliable power measurement requires calibration of that directional power measurement through water calorimetry, along with determining a water-cooling flow rate. In addition, the single-colour pyrometer was calibrated via the two-colour pyrometer.

\subsubsection{Microwave leakage detection}

To ensure the safety in the system, a simple precursor RF receiver antenna with a diode between the arms was built to produce a rectified DC response to leaked microwave radiation, shown in Figure 4.11. The antenna had not been calibrated, but was tested with standard microwave ovens where the leakage from the side of the door was enough to 'max' out the needle on the RF receiver. Since commercial microwaves are developed to be microwave safe, the purpose of the RF receiver was to find sources of leakage, as a qualitative measurement and general safety indication.

For the microwave furnace to be a useful tool in heating materials it must be safe to operate. For the laboratory scale microwave furnace, the amount of microwave leakage was measured with an ERD (Electromagnetic $\underline{\text { Radiation }}$ Detector). The detector is a simple half-wave dipole antenna [88]. The ERD then scales the voltage generated across the antenna arms to a calibrated logarithmic scale, similarly to the AD8317 RF-DC detector boards to be discussed in the next section. The leaked microwave power is measured by holding the ERD next to each possible source of microwave power leakage in the experimental setup. The orientation of the ERD is important in measurement, so all three orthogonal orientations are checked for each potential source of 


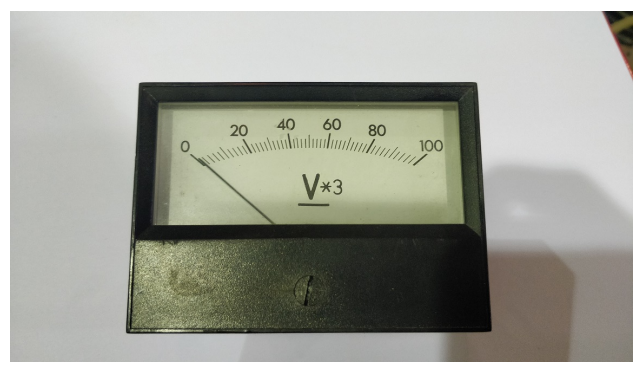

(a) Face of RF receiver

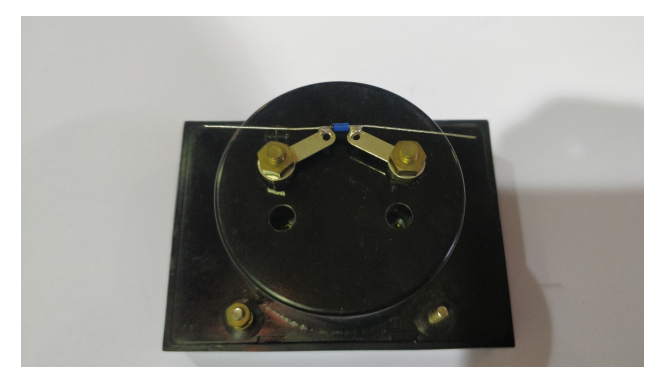

(b) Back of RF receiver

Figure 4.11: RF receiver for initial qualitative microwave leakage measurements. The needle moved when microwave radiation interacted with the antenna on the back of the RF receiver. The functionality and sensitivity of this device was tested by observation of microwave leakage from a commercial microwave oven at the on-site cafeteria.

microwave leakage along the transmission line.

The ERD was factory calibrated previously to a wide range of microwave frequencies. Each LED (Light Emitting Diode) on the ERD corresponds about to an increased order of magnitude of microwave power. The highest sensitivity of $2.45 \mathrm{GHz}$ microwave radiation was approximately 3 LED's. This corresponds to a background field intensity of $0.25 \mathrm{~mW} / \mathrm{mm}^{2}$. There was no significant increase of microwave leakage above the background level of the ERD from the rest of the microwave system. The maximum microwave power that was measured was 8 LED's from magnetron launch connection. From Figure 4.12b,

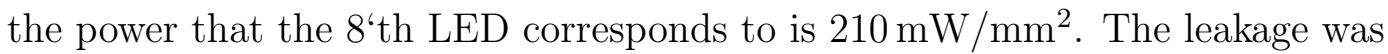
minimised (i.e., reduced to ambient levels) by tightening the screws holding the magnetron to the transmission line.

Human general public long term exposure limit is $4939 \mathrm{~mW} / \mathrm{mm}^{2}$ [80] calculated via $U=\frac{1}{2} c \varepsilon_{0}|\vec{E}|^{2}$. The greatest microwave leakage was due to an imperfect connection of the base of the magnetron to the WR340 waveguide launch in Figure 4.3a. The ERD showed that the system was successfully designed to be microwave-safe during operation.

\subsubsection{AD8317 RF-DC detectors}

To validate the electromagnetic simulation of the COMSOL model, a physical directional power measurement is essential. A key measurement for the model 


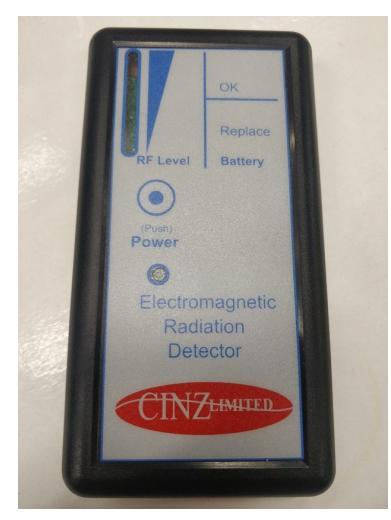

(a) The ERD detector.

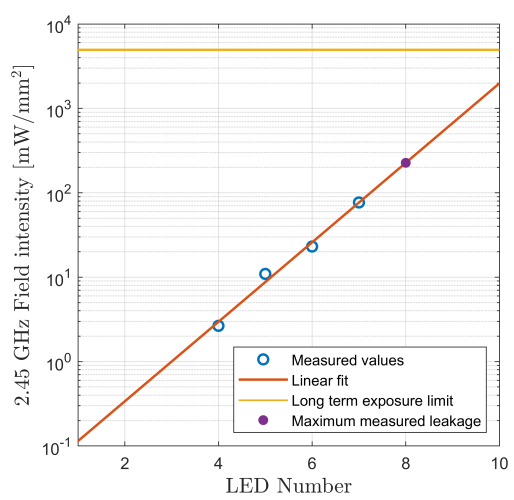

(b) LED light number against microwave power.

Figure 4.12: ERD detector, and previously-calibrated power measurements for quantitative microwave leakage measurements. A linear fit (in $\mathrm{dBm}$ which is a logarithmic power measurement unit, relative to $1 \mathrm{~mW}$ ) was employed to find the power measurement beyond the calibrated range.

investigation process is the acquisition of the reflection coefficient $r$ of the cavity, defined as the ratio of reflected power from the cavity over the power incident to it, and how that reflection coefficient changes as the materials in it absorb microwave power. The reflection coefficient is significantly affected by the interaction of the ironsand with microwave energy; i.e. the temperature dependence of $\varepsilon_{r}$ and $\mu_{r}$. Connected to the coaxial lines on the dual-directional coupler in Figure 4.7 are AD8317 RF-DC detectors, pictured in Figure 4.13.

The specific RF receiver type used in this work is an Analog Devices AD8317 $55 \mathrm{~dB} \log$ detector, which turns RF power into a logarithmically scaled DC voltage. In turn, this voltage is measured with a NI (National Instruments) USB-6001 DAQ (Data Acqusition) card. According to the AD8317 RF-DC detector board datasheet found online, it has an input range of time averaged $-5 \mathrm{dBm}$ to $-55 \mathrm{dBm}$ microwave power if the frequency is near $2.2 \mathrm{GHz}^{4}$. The resulting output DC voltage that the chip produces ranges from $1.6 \mathrm{~V}$ to $0.4 \mathrm{~V}$. This results in a primarily linear power scale of $2.4 \mathrm{mV} / \mathrm{dB}$. This voltage measurement is acquired and logged via LabVIEW software. The AD8317 RF-DC detectors therefore allow for analysis of the directional power passing through the dual-directional coupler transmission line component.

There were various stages to calibrating the voltage measurements from the

\footnotetext{
${ }^{4}$ The detector has a higher power detection range at a lower frequency.
} 


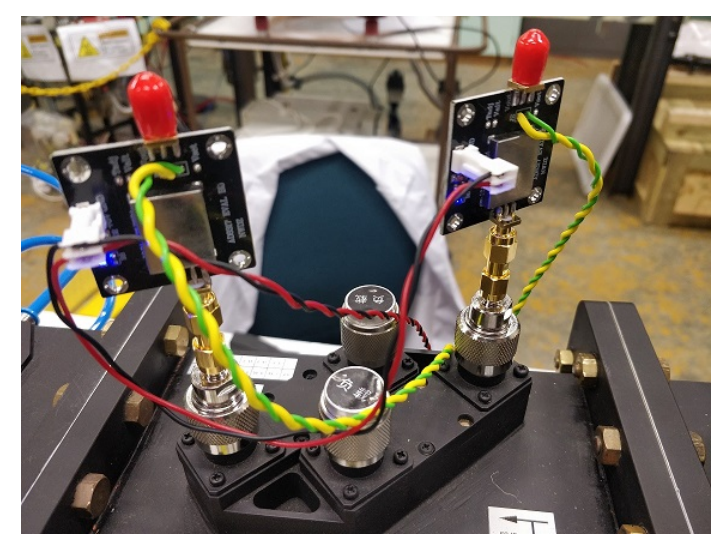

Figure 4.13: AD8317 RF-DC detector boards attached via adapters to the coaxial connectors on the dual-directional component of the transmission line.

AD8317 RF-DC detectors to RF power measurements. The NI DAQ was used to acquire signals at the highest acquisition rate for the piece of equipment, $10 \mathrm{kHz}$. There were two unexpected types of signals observed during measurement, the first of which was a rectified $100 \mathrm{~Hz}$ mains 'hum' enveloping the acquired signal. The time-average of the enveloped signal was presumed to be the same as the DC power measurement. The maximum power that could be measured by the RF-DC detectors was $-5 \mathrm{dBm}$, which corresponded to $316 \mathrm{~W}$ when the $60 \mathrm{~dB}$ coupling from waveguide to coaxial line was included. This resulted in a 'clipped' power measurement (as the peak power output by the magnetron over one cycle was well over $316 \mathrm{~W}$ ). Averaging this measurement would not well represent the RMS microwave power. The solution to this was to add extra $20 \mathrm{~dB}$ attenuators, which increased the maximum instantaneous power measurement to $31.6 \mathrm{~kW}$ (i.e., changed the power measurement range to between -35 to $15 \mathrm{dBm}$, before inclusion of the $60 \mathrm{~dB}$ coupling from the dual-directional coupler).

Due to the aliased signal time-averaging, the signal still gave nonphysical results. The source of the aliased signal then needed to be found, and those signals filtered from the measurement. The aliased signals were measured with a Tektronix TPS $2014100 \mathrm{MHz}$ oscilloscope. The oscilloscope was connected to an exposed section of the output voltage lines of the AD8317 detectors. Using the Fourier-transformed view of the oscilloscope while all equipment is disconnected from power, various high frequency ( $>50 \mathrm{kHz}$ ) background signals were observed. These signals were suspected to come from other equipment used in the laboratory space. Then, the power supply was connected but not turned on. A picture of the resulting FFT indicates the presence of a significant 
$150 \mathrm{kHz}$ signal as shown in Figure 4.14.

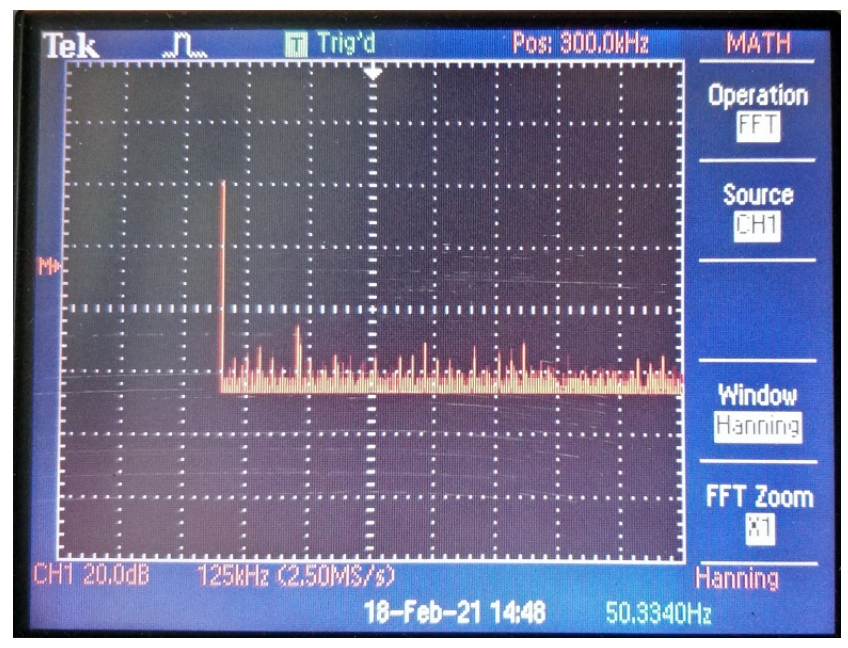

Figure 4.14: Oscilloscope screen while connected to AD8317 board output lines. The $150 \mathrm{kHz}$ signal was clearly present in this view of the signal. In addition, a $50 \mathrm{~Hz}$ mains hum was noted by the oscilloscope.

During powering of the magnetron, electrical noise (spurious high frequency signals) was present across the entire frequency, spectrum similarly as in Figure 4.14. The $150 \mathrm{kHz}$ signal dominated this spectral noise. To remove this range of high frequency aliased signals, a $1 \mu \mathrm{F}$ capacitor was placed in parallel with the ground and the detector wires of the NI USB-6001. The capacitor was used as a low-pass filter by producing a low impedance path for the high frequency signals, essentially removing their contribution to the acquired data. The internal resistance of the chip was used as the resistive load in this RC low-pass filter. However, the resulting amplitude of the acquired data was reduced. The bias voltage of the detectors was unchanged and it was assumed that the voltage measurement range remained at $1.2 \mathrm{~V}$. The internal voltageto-RF-power calibration of the boards was invalidated by the filtering of some of these high frequency signals. This indicated that some significant microwave power was carried by various high frequency $(\approx 100 \mathrm{kHz})$ signals, produced by the magnetron. The power associated with those signals was no longer measured as they were filtered out of the measured data. Water calorimetry was then used to calibrate the adjusted voltage-to-RF-power scaling with a physical power measurement from the heating of water passing through the terminator. 


\subsubsection{Water calorimetry}

The magnetron produces some amount of microwave power written as $P_{f w d}$. The cavity does not absorb all of this microwave power, so some power is reflected. For the purpose of the calibration, it was assumed that reflected power from the cavity $P_{b w d}$ is assumed to be equal to the power deposited in the water cooling loads in the terminator. The reflected power $P_{b w d}$ could therefore be calibrated via the temperature rise $\Delta T$ of the water via

$$
P_{b w d}=\frac{\Delta m}{\Delta t} c_{p} \Delta T
$$

The room temperature heat capacity of water is well known; $c_{p, w a t e r}=$ $4180 \mathrm{~J} / \mathrm{kgK}$, varying slightly with temperature. The output water temperature was measured by NI USB-TC01 with a K-type thermocouple, as before mentioned it was wrapped in PTFE tape to improve thermal isolation. The terminator is the first water-cooled component along the water-cooling line. The input water temperature was found to remain constant (i.e. at room temperature) throughout calibration. To measure the microwave power deposited into the water, knowledge of the mass flow rate $\Delta m / \Delta t$ is essential. The water flow rate is measured with an RS PRO Flow Meter via LabVIEW software, placed in line with the water cooling during operation. A $5 \mathrm{~V}$ supply line was connected to the flow meter, again controlled by the DAQ. A $2.2 \mathrm{k} \Omega$ pull-up resistor was connected between the $5 \mathrm{~V}$ line input and signal output. The water flowing through the flow meter pushes a free-running turbine, with over-moulded magnets where the rotation rate is measured by a Hall effect sensor. The flow meter then produces a digital signal of $5 \mathrm{~V}$ pulses at a frequency proportional to the flow velocity. The pulse frequency to mass flow rate proportional constant is referred to as the K-factor ${ }^{5}$. To determine the $\mathrm{K}$-factor for the flow meter used in this work, water flow rate calibration was done. A water hose with $3 / 8$ " OD was connected from a 3 atmosphere water supply through the flow meter, and into a bucket. The weight of the bucket was measured before the tap was turned on, then measured again after 1 minute, after which the tap was turned off. A variety of valve positions were chosen for the tap, resulting in different water flow rates into the bucket and a range of pulse frequencies as produced by the flow meter. The total number of pulses

\footnotetext{
${ }^{5}$ The datasheet of the RS PRO 511-4772 flow meter quotes a K-factor of approximately 1120.
} 
was compared against the total weight difference of the bucket. The flow and tick rates are compared by dividing each by the flow duration in Figure 4.15.

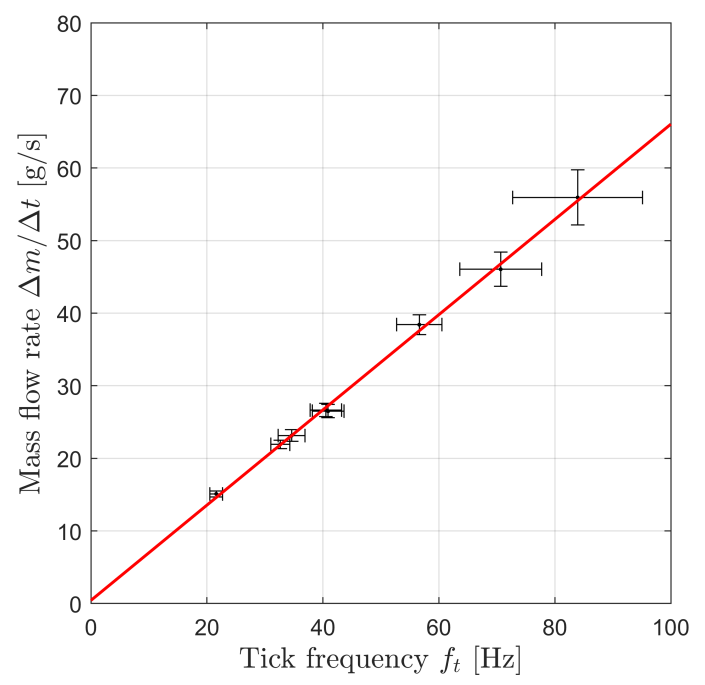

Figure 4.15: Pulse rate to mass flow calibration. It shows an excellent linear relationship $\Delta m / \Delta t=0.66 f_{t}+0.43$ between tick rate and mass flow rate. Note that the linear relationship is extrapolated to below 20, and above $83 \mathrm{~Hz}$.

Thus, the flow rate is given in terms of the tick rate $f_{t}$ by $\Delta m / \Delta t=$ $0.66 f_{t}+0.43$. Extrapolations below this flow rate suffer from tick quantisation, where the rate of ticks limited the approximate flow rate measurement accuracy.

The next calibration required is to calculate the power deposited into the water in the terminator, with (Eq. 4.3.1). The input power to the magnetron was controlled from $0 \%$ to $100 \%$ in discrete steps. After turning the dial up, a one minute delay allowed the water to reach a steady-state temperature. The reflected power signal measured by the AD8317 RF-DC detectors was calibrated against the power deposited into the water as it passed through the terminator. There were two core assumptions in this approach. The first was to assume that the thermal losses such as conductive losses along the water-cooling tubing are small compared to the heat energy carried by the water. The second was that effectively all microwave power incident on the terminator was used to heat the water (thus no resulting power would propagate towards the magnetron through the circulator). A calibration curve for various measured voltage-to-power produced by the AD8317 board was then calculated, and is shown as the blue line in Figure 4.16. Some post-processing must be done to the data acquired by the RF-DC detectors. To succinctly present the 
processing procedure, the following formula was employed;

$$
V_{b w d}=\log _{10}\left(\frac{1}{N} \sum_{i=1}^{N} 10\left(V_{b w d, i}^{\prime}-V_{b w d, \text { max }}^{\prime}\right)\right)
$$

with number of samples $N=10000$ with acquisition rate $10 \mathrm{kHz}$. A singe backward voltage measurement is denoted as $V_{b w d, i}^{\prime}$. This voltage measurement is relative to some reference voltage $V_{b w d, \max }^{\prime}$ associated with a zero power measurement value. The time-averaged voltage value was averaged over 10000 measurements. The calibration was done with respect to the reference voltages for $V_{f w d, i}^{\prime}$ and $V_{b w d, i}^{\prime}$. These reference voltages are $V_{f w d, \max }=1.53 \mathrm{~V}$, and $1.50 \mathrm{~V}$ for $V_{b w d, \max }=1.50^{6}$.

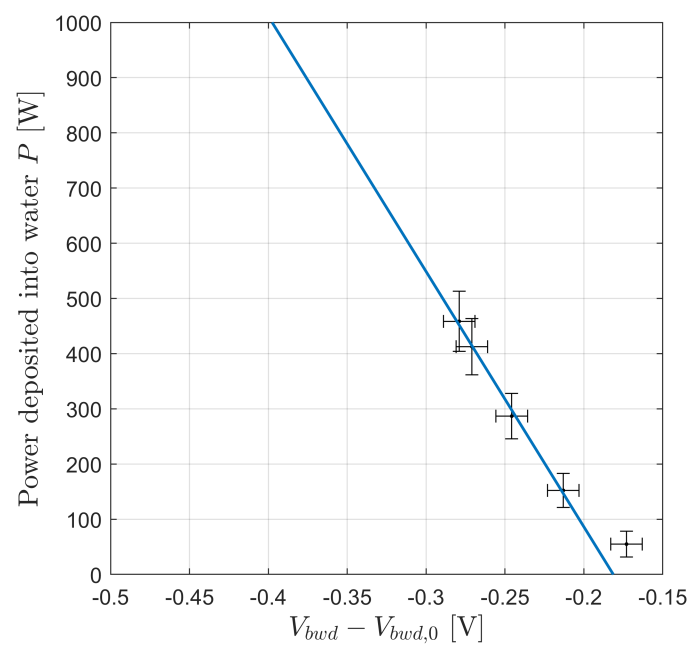

Figure 4.16: Plot of measured backward voltage against power deposited into water.

It is also noted that at low input powers to the magnetron, most of the power supplied power does not linearly increase microwave production. The 'cut off' input power beyond which the microwave production appeared linear was when the controller set the power supply output to over $30 \%$ of maximum power. Since the calibration is mainly of interest when large microwave powers are transferred, those low input power measurements $(0-30 \%)$ were not included in the calibration. The forward voltage measurements were assumed to adhere to the same calibration as the reverse voltages did. The linear relationship between

\footnotetext{
${ }^{6}$ It was verified that they are board-specific reference voltages by swapping AD8317 board and dual-directional coupler ports, then repeating the measurement.
} 
voltage measurement and power deposited into the terminator component (as seen in Figure 4.16) was given by $P=-4624 V-839$ with $R^{2}=0.997$. The accuracy of this calibration was mainly limited by the minimum tick rate of the flow meter. Thus, the power as measured by the RF-DC AD8317 boards was calibrated with the power deposited into flowing water through the water load in the terminator. Then, since both forward and backward power measurements are calibrated via water calorimetry, the reflection coefficient $r$ of the cavity is determined in terms of a ratio of the backward-over-forward power. This reflection coefficient is written as

$$
r=\frac{P_{b w d}}{P_{f w d}}
$$

This ratio between backward and forward power can also be expressed in terms of decibels via

$$
r=10 \log _{10}\left(\frac{P_{b w d}}{P_{f w d}}\right)
$$

This is the most common form of representing reflection losses in microwave electronics, and is the way by which transient microwave cavity reflection coefficient data is presented in Chapter 5 .

\subsubsection{Pyrometers}

In addition to measuring the directional power in the system, a temperature measurement of the heated ironsand is essential. Standard thermocouples do not suit these types of temperature measurements as the thermocouple wire will act as an antenna for microwave power [65]. This will damage the thermocouple, and result in potentially increased microwave leakage. Optical pyrometers were the ideal solution to measuring temperatures in a microwave furnace. To allow for those temperature measurements, direct optical access to the bored BN tube which contained the ironsand was required. This required the addition of two optical access holes in the side of the resonant cavity, and safe geometric implementation of these holes is described in Chapter 3.6.

Two infrared optical thermometers were used in this research. The first is a single-colour pyrometer from Micro Epsilon, specifically the CTLaserM2 with CF2 lens pyrometer, and a two colour pyrometer, the QKTRD-4485 with TR 2 pyrometer. The CTLaserM2 is sensitive to $\lambda=1.60 \mu \mathrm{m}$ with a temperature 
range $T \in(490,2000)^{\circ} \mathrm{C}$. The QKTRD-4485 two colour pyrometer is sensitive to $\lambda_{1}=0.97 \mu \mathrm{m}$ and $\lambda_{1}=1.05 \mu \mathrm{m}$ with a temperature range $T \in(800,2000)^{\circ} \mathrm{C}$. The single colour pyrometer has a lower minimum temperature, but is not automatically emissivity corrected. An emissivity correction is important due to the thermal radiation profile (Eq. 2.3.2) of BN used in this work [89]. Two colour pyrometers solve this emissivity issue by measuring the intensity of two separate (but still close) wavelengths of emitted light. The pyrometer then takes the ratio of the intensity of light of those two wavelengths and calculates a unique temperature associated with that ratio. Pictures of the pyrometers are included as Figures 4.17a and 4.17b.

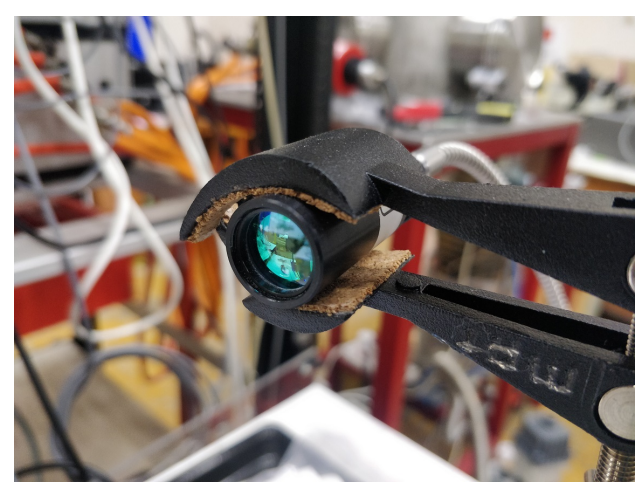

(a) Dr. Maurer Optoelektronik two colour pyrometer, QKTRD-4485.

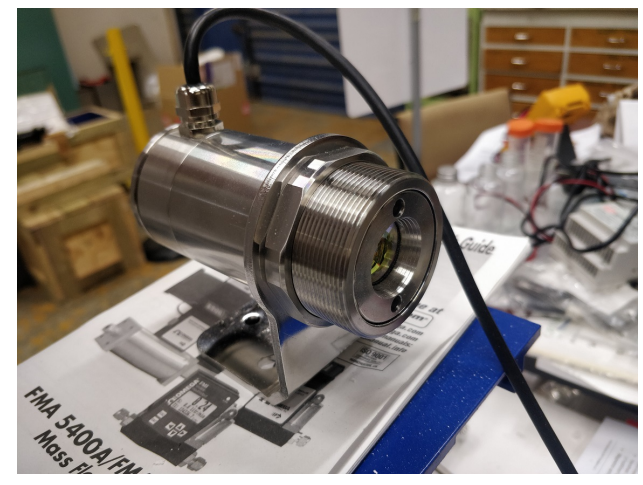

(b) Micro Epsilon single colour pyrometer, CTLaserM2, CF2 lens.

Figure 4.17: Pictures of 4.17a two colour QKTRD-4485, and 4.17b single colour CTLaserM2 pyrometer measurement heads.

In this research, the two-colour pyrometer was used to calibrate the temperature measurement of the single-colour pyrometer. This emissivity calibration was then extended to the entire temperature range of the single-colour pyrometer. This emissivity calibration was then performed with the microwave furnace using a small BN tube (ID $10 \mathrm{~mm}$, OD $15 \mathrm{~mm}$ ) packed with TTM ironsand, and centred in the microwave cavity. The cavity and tube were purged with $\mathrm{N}_{2}$ gas, and the pyrometers activated after being focused on the surface of the BN tube. The BC waveguide ends were packed with Kaowool to minimise convective heat losses. The magnetron was then activated at maximum power for $\approx 10$ minutes without tuning the stubs in the three-stub tuner. Once a steady state temperature had been reached, the stubs were then moved to improve the resonance performance of the furnace. Once a final steady-state temperature was reached 
at the measurement spot, the magnetron was then turned off, so the heated ironsand and BN rapidly radiatively cooled. Once the spot temperature fell outside the temperature range of the pyrometers, all measurements were ended. A second order polynomial is used for the temperature correction function of the single colour CTLaserM2 pyrometer as

$$
T_{\text {Ratio }}=3.6981 \cdot 10^{-4} T_{\text {Single }}^{2}+0.5733 T_{\text {Single }}+223.3014
$$

This was calculated in MATLAB from regression analysis with $R^{2}=0.9991$. Both temperatures are measured in ${ }^{\circ} \mathrm{C}$ and visualised as Figure 4.18.

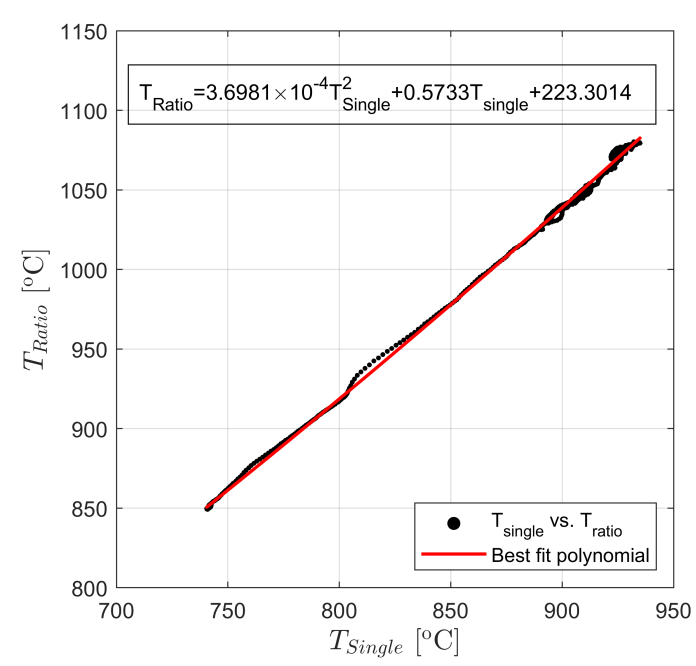

Figure 4.18: Comparison of spot temperature measurements made by single colour CTLaserM2 pyrometer, and two-colour QKTRD-4485 pyrometer. For this calibration, the emissivity of $\mathrm{BN}$ is assumed to be constant near the measurement wavelengths $\left(\lambda_{1}=970 \mathrm{~nm}, \lambda_{2}=1050 \mathrm{~nm}\right)$ for the QKTRD-4485 pyrometer.

Minor deviations from this curve were observed during heating. From the instrument error, a $1 \%$ of measurement value and $5^{\circ} \mathrm{C}$ error in temperature correction multiplied with the calibration is used in further measurements. This comes from the two instrument errors, as the temperature as measured by the single-colour pyrometer needed to be calibrated by the two-colour pyrometer. The factory measurement error of the two-colour pyrometer is $0.5 \% \pm 1^{\circ} \mathrm{C}$ and the factory error of the single-colour pyrometer is $0.3 \% \pm 3{ }^{\circ} \mathrm{C}$. The correction polynomial in (Eq. 4.3.5) is extrapolated to the entire temperature range of the CTLaserM2 pyrometer $\left(T_{\text {single }} \in(490,2000)^{\circ} \mathrm{C}\right)$. This assumption is suitable if 
the slope of the emissivity between $970 \mathrm{~nm}$ and $1050 \mathrm{~nm}$ of $\mathrm{BN}$ is assumed to not change significantly between $550^{\circ} \mathrm{C}$ and $850{ }^{\circ} \mathrm{C}$.

\subsection{Explorational experimental findings}

This section details some supplementary qualitative findings of the behaviour of TTM ironsand during initial experiments in various crucibles in the microwave cavity. Firstly, plasma was produced within the cavity when there were sharp crucible edges or open surfaces of loose TTM ironsand. This resulted in partial melting of many crucibles, and often of the ironsand itself. Many Alumina crucibles were destroyed due to the thermal stresses applied by the intense heating by the microwaves. Some BN crucibles were also cracked due to highly rapid heating and cooling of material ${ }^{7}$.

\subsubsection{Plasma production}

Many times throughout this research, microwave plasma was produced as a result of excellent impedance matching between magnetron and microwave cavity. When this was achieved, the field amplitude of the microwaves in the cavity increased drastically. Microwave plasma was produced at sharp corners of objects in the cavity as a result. This effect was most pronounced in the small 'boat'-shaped crucibles used early on in this work. This can be seen in Figure 4.19.

A plasma is a gas of ions and electrons [90,91]. Microwave plasma is produced when the space through which the microwaves propagate exceeds its breakdown voltage. This breakdown voltage is the point at which the valence electrons in a gas are given enough energy to become free electrons [92]. For air (which is primarily composed of $\mathrm{N}_{2}$ gas) the breakdown voltage is $34.5 \mathrm{kV} / \mathrm{cm}$ [93] at 1 atmosphere. At the interface between a conductor and air, the electric field lines must enter the conductor perpendicularly ${ }^{8}$ to the interface. If a conductor has a sharp point, then these field lines focus on that point, easing the breakdown voltage condition to be met.

\footnotetext{
${ }^{7}$ Often, a faintly burnt scent could be smelled from the furnace. This was usually the first indication that the ironsand was heating, even before the ironsand transitioned past its Curie temperature.

${ }^{8}$ This is true when the conductor is a perfect conductor.
} 


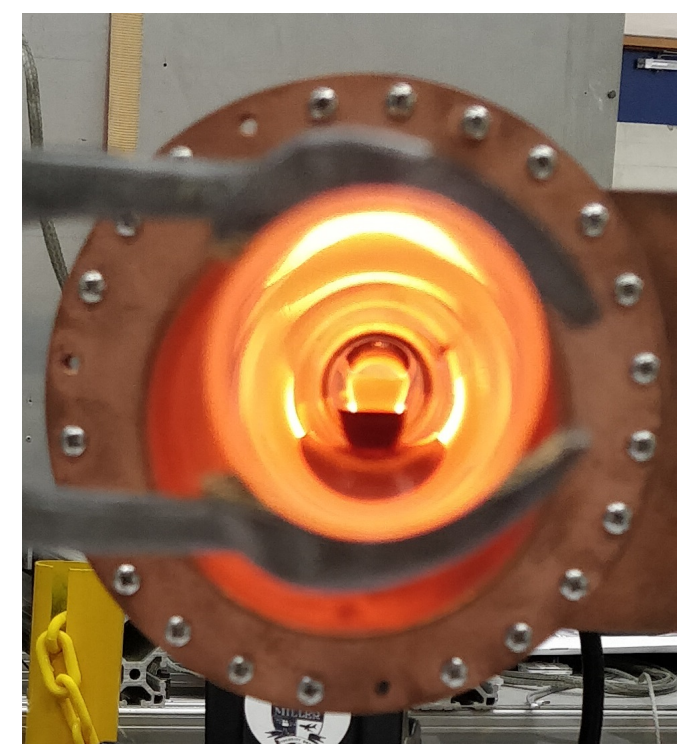

(a) High temperature ironsand

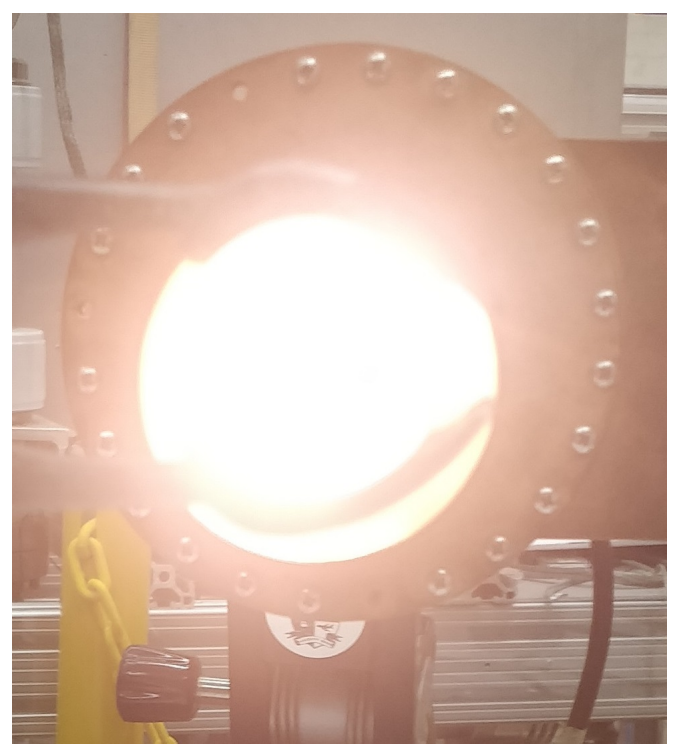

(b) Plasma ignition

Figure 4.19: Before-and-during pictures of plasma ignition in microwave cavity with Alumina boat filled with ironsand. A loud low-pitch sound was produced while the plasma was lit. This was due to the $50 \mathrm{~Hz}$ mains 'hum', powering the magnetron, resulting in plasma pulsing.

Thus, a plasma was produced when the system was well matched (i.e. reflected microwave power $r$ is minimised) and the system is optimally heated (see Figure 4.19b). The microwave plasma was observed to occur very near to the surface of the TTM ironsand in the crucible, where large thermal gradients in materials may have promoted internal sparking. This internal sparking was observed as Lichtenberg figures (discussed in more detail in Chapter 4.4.2). The temperature of the container Alumina boats and BN tubes decreased when a plasma was ignited, as all microwave power was focused into maintaining the microwave plasma, due to its high conductivity. Plasma is generally an excellent conductor, due to the high mobility of free charge carriers. Thus the conductive losses dominate the power losses in the cavity. It is also noted that the impedance of the cavity also suddenly changed due to the presence of the plasma. The plasma thermally radiates intensely, and heated the material which it is closest to, which was generally a microwave transparent crucible. This results in permanent damage to the crucible, as in Figure 4.20.

The melting point of Alumina is $2072{ }^{\circ} \mathrm{C}$ [94], implying the temperature of the plasma greatly exceeded the melting temperature of Alumina. The rapid 


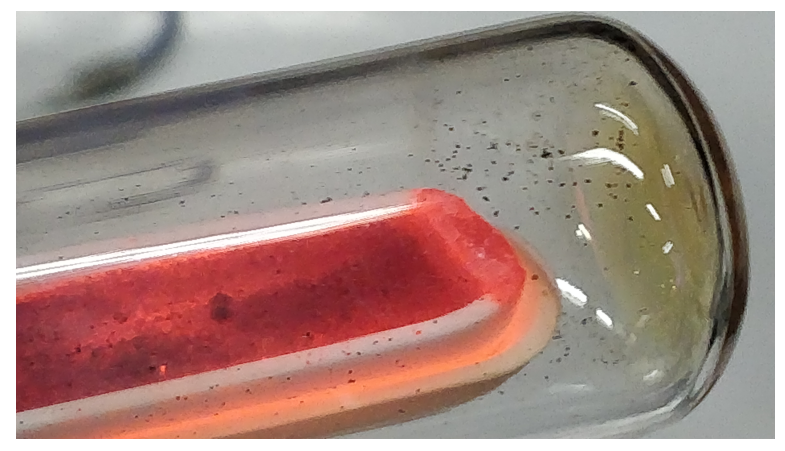

Figure 4.20: Red-hot glowing TTM ironsand and melted-end of alumina crucible in closed-ended quartz tube. Some browning of the end of the quartz tube is seen and is due to plasma deposition of materials onto the tube.

cooling from this high temperature resulted in crystallisation of material in the melted section, shown in Figure 4.21.

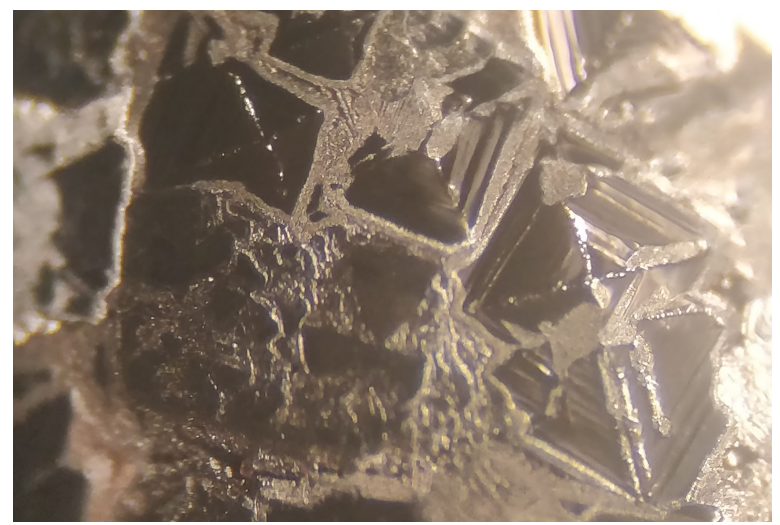

Figure 4.21: Crystallised surface of melted section of Alumina-ironsand from Figure 4.20 after rapid radiative cooling, viewed under microscope. The crystal structures shown here match the macroscopic crystalline structure of pure magnetite, meaning that the ironsand was also fully melted.

A clear crystallisation occurred after radiative cooling of the ironsand and alumina 'boat'. The crystal produced was suspected to be a combination of TTM, TTH ( Titanohematite) (due to the oxidation of the TTM at high temperatures), and alumina.

\subsubsection{Lichtenberg figure production}

A Lichtenberg figure is the result of dielectric breakdown within an insulator [95-97]. They are essentially a 'lightning-strike' of electric discharge within the 
insulating material, and large-scale versions are most often seen when lightning strikes the ground, for example fusing mineral grains in desert sand. That case of electric discharge is more specifically known as a fulgurite. In a few experiments, Lichtenberg figure structures were produced within the ironsand, displayed in Figure 4.22. These Lichtenberg figure arms were extremely delicate once exposed. The surface was partially oxidised and melted at one section. This was produced via plasma ignition.

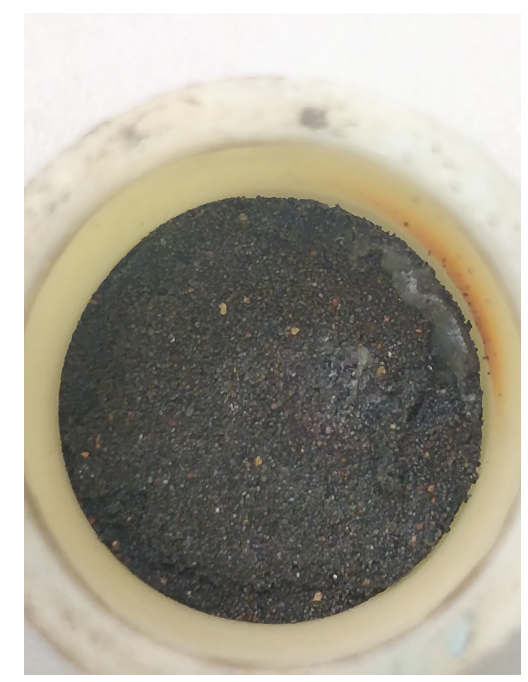

(a) Top-down picture of heated ironsand.

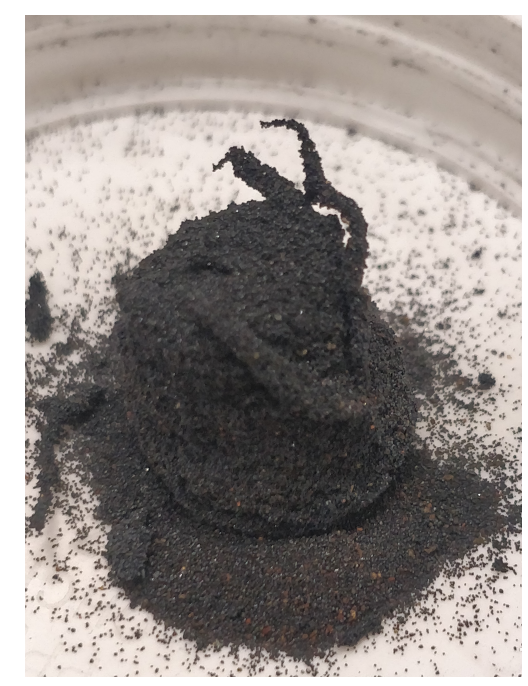

(b) Lichtenberg figures produced in ironsand.

Figure 4.22: Lichtenberg figure produced in body of loose ironsand in Alumina crucible after the loose ironsand was carefully tapped off.

The production of the Lichtenberg figure arms was often observed, but never reliably produced. An explanation for this effect is that when the plasma ignites (and the sand has not sintered), a lightning strike-like effect occured within the TTM ironsand. Since producing Lichtenberg figures was not the purpose of the microwave furnace, it was important to have no sharp edges within the microwave cavity itself, so to minimise the chance of producing a microwave plasma. This then minimises the production of Lichtenberg figures.

\subsubsection{Partial ironsand sintering}

Sintering is the process of compacting and forming a solid mass of material (in the context of this research the ironsand), via heating, while below the 
melting point of the material [98]. In the case of the small BN tube used in the research, it was also of interest to produce sintered 'rods' of TTM ironsand as an alternative application for a microwave furnace. These rods are easier to process since they remain porous even after sintering, so gas can be passed through the sinter without venting through, or fluidising the bed of ironsand. Rods were often successfully produced, however often at the cost of the crucible itself. Nonetheless, the TTM ironsand was sometimes safely extractable while retaining an intact crucible. By increasing the wall width of the bored BN tube, the lifetime and reusability of the rod remarkably improved. The inner walls of those crucibles were not visibly spoiled by the heated TTM ironsand. Two pictures follow of a partially sintered/melted/oxidised 'rod' of TTM ironsand. The first (Figure 4.23) is a picture of a cross section from the middle of the solidified ironsand, and the second (Figure 4.24) is a picture of the top-end of the solidified ironsand.

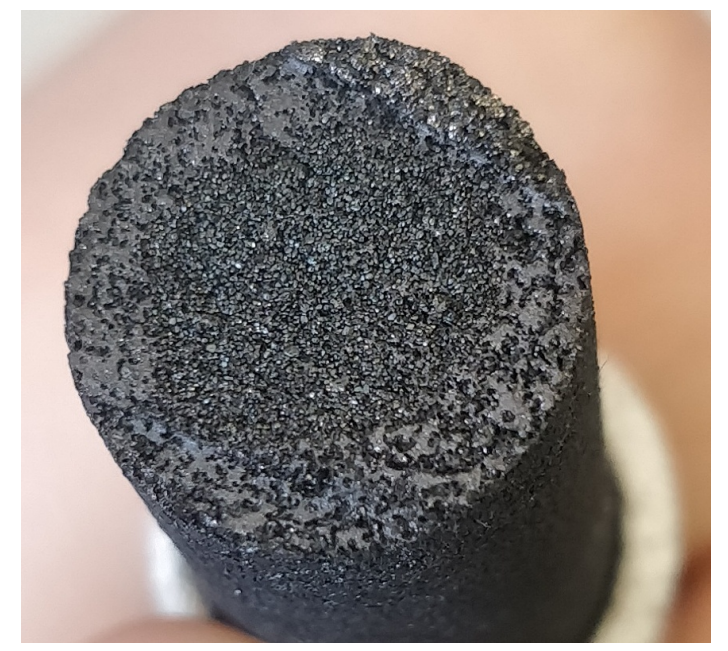

Figure 4.23: Cross section of extracted rod of partially-sintered TTM ironsand. The diameter of this rod is $14 \mathrm{~mm}$. There are two phases of ironsand; the outer which is partially melted, and the interior of the tube which is effectively sintered.

This partial sintering shows another potential application of the microwave furnace, as the heating along with electromagnetic mode placement can be controlled by different cavity designs. However, this is not directly the focus of this work. Further research could develop this approach as an alternative continuous method to producing ironsand sinter for other applications. 


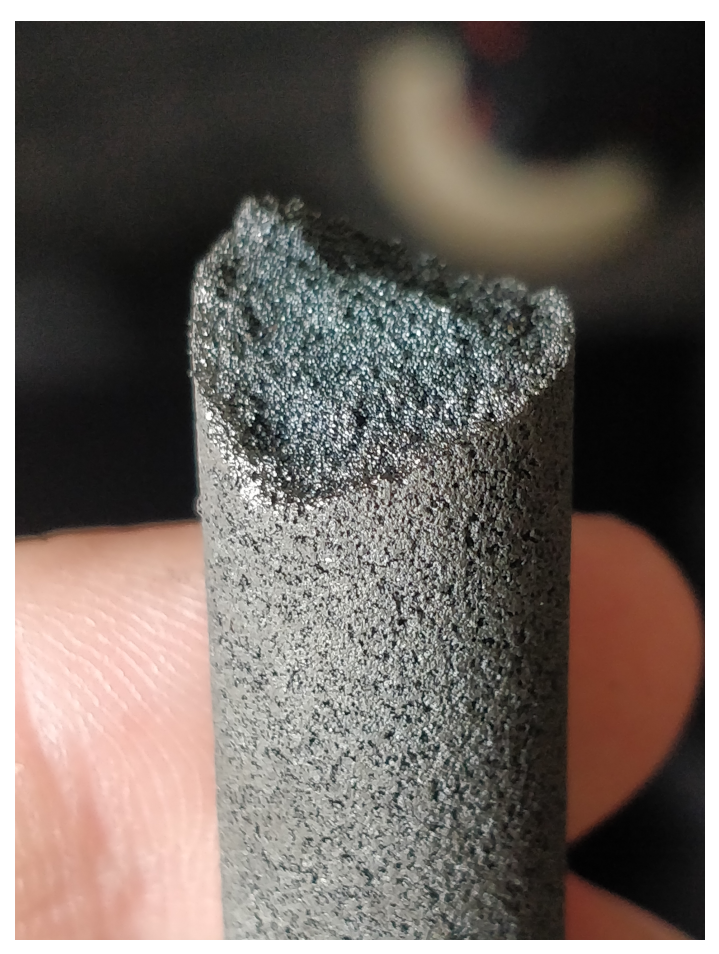

Figure 4.24: End-piece section of extracted rod of partially-sintered TTM ironsand. The diameter of the rod is $14 \mathrm{~mm}$. The internal section of this appears partially sintered. A blue hue in the ironsand is observed at the end of this partially-sintered ironsand column.

\subsubsection{XRD analysis}

XRD (X-Ray Diffraction) was employed to review if any significant chemical species changes occurred during the heating of the ironsand. Researchers Ao Zhang (Robinson Research Insitute) and Martin Ryan (Callaghan Innovation) performed the XRD measurements and helped with analysis of the following results. A reference sample of raw unheated ironsand was used as a control measurement. Two heated samples were presented: one which was heated under a $\mathrm{N}_{2}$ gas sheath, whilst the other was heated under air under the same experimental procedure set out in Section 4.5. The temperature reached by the surface of the bored $\mathrm{BN}$ rod of this sample was $650{ }^{\circ} \mathrm{C}$, over 10 minutes.

The heated samples of the ironsand showed some variation in relative peak intensity for the TTM phase (the peaks at $51^{\circ}$ and $90^{\circ}$ as seen in Figure 4.25). However, no increase of the intensity of the TTH phase was observed. In addition, it was observed throughout this work that when ironsand was heated a second time in the furnace, it reached steady-state temperatures much 


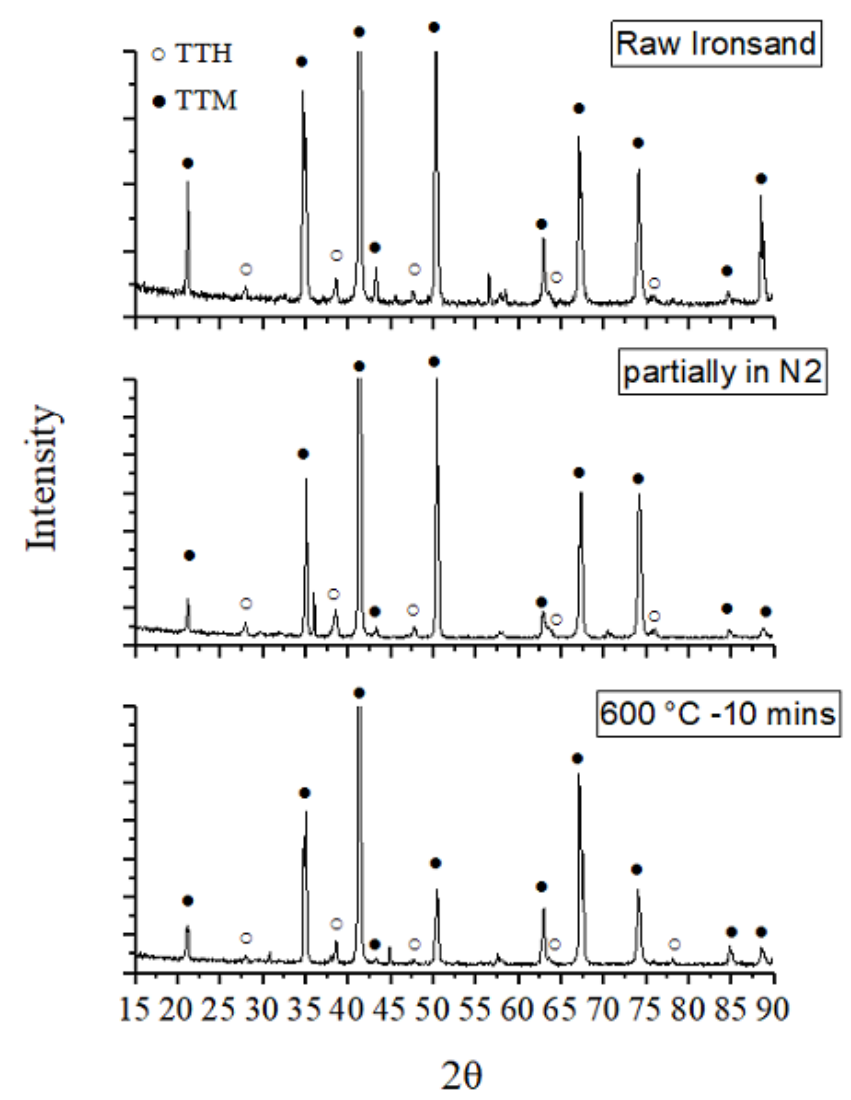

Figure 4.25: XRD scans of ironsand used in this work, compared with raw ironsand. Patterns indicate that the microwave-heated ironsand in the cavity had not significantly oxidised throughout heating. The dots indicate the presence of TTM, and the circles indicate presence of TTH. The x-ray source used to obtain these diffraction patterns was Co-60.

faster than ironsand that had not been previously heated. This could be due to the expulsion of impurities in the ironsand, allowing for significantly improved microwave heating at lower temperatures. This indicates that purifying (or preheating) the ironsand could greatly improve the performance of the microwave furnace. As this purification stage is generally an essential step to the reduction process, regardless of whether a microwave furnace is used, a microwave heating method of rapidly 'boosting' the temperature of ironsand may show promise. This however was a qualitative result, and would require further research for a more detailed analysis. 


\subsection{Experimental procedure}

This section details the process of taking test measurements of the experimental system, along with a diagram of the experimental setup, shown in Figure 4.26. Optical pyrometers measure the transient heating $T(t)$ of the bored BN rod in the centre of the cavity (which is in direct thermal contact with the ironsand). The calibrated AD8317 boards on the RF-DC receivers measure the directional power propagating within the system to calculate the transient reflection coefficient of the cavity $r(t)$ with (Eq. 4.3.4) in $\mathrm{dB}$. The temperature near the end of the BN crucible (at the ends of the BC waveguide) $T_{B C}(t)$ is measured by K-type thermocouple with the National Instruments USB-TC01 and automatically acquired via LabVIEW software. All measurements are compared to the initial model and used to infer refinements to that model, shown in Chapter 5.2.

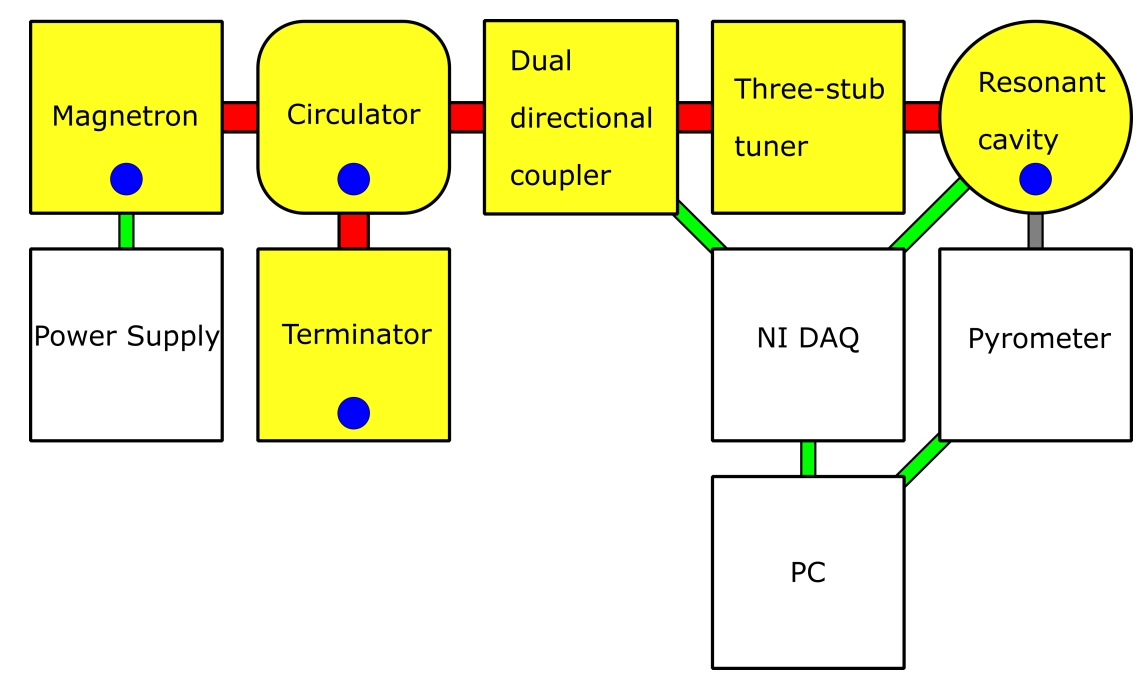

Figure 4.26: Diagram of experimental setup. Microwave power is contained in the yellow blocks, the red sections being WR340 waveguide connections. Green sections are connections between various electronics. The grey connection is the optical access of the pyrometer to the resonant cavity. The blue dots on the transmission line components are where water-cooling is employed.

The experimental process was extensively explored throughout the thesis, and it was found that the electronic properties significantly varied around the 
Curie temperature of the ironsand ${ }^{9}$. Thus, the experiment used to refine the computational model was made as simple as possible to exemplify this effect. The process in taking a measurement to infer refinements to the computational was as follows:

1. wear safety glasses, laboratory coat, closed footwear,

2. fill BN crucible with TTM ironsand and centre it in the cavity,

3. turn on electronics, except power supply,

4. ensure $\mathrm{N}_{2}$ gas flow ${ }^{10}$ and water cooling valves are open,

5. focus the pyrometer on BN tube surface through optical access holes,

6. wrap thermocouple wire around the $\mathrm{BN}$ rod at end of $\mathrm{BC}$ tube, while ensuring that the end is in contact with $\mathrm{BN}$ rod,

7. fill ends of $\mathrm{BC}$ waveguides with Kaowool to minimise convective thermal losses,

8. ensure all stubs of three-stub tuner are fully retracted,

9. turn on power supply at minimum input power for 30 minutes,

10. begin acquisition of temperatures and directional power ${ }^{11}$,

11. turn on power supply at maximum power for 600 seconds,

12. turn off power supply,

13. after the surface of the BN tube has cooled below the minimum temperature measurement of the pyrometer, turn off all acquisition data and close $\mathrm{N}_{2}$ gas valve.

This scheme has been shown to exhibit highly repeatable results, exemplifying the temperature variation, specifically the significant increase of the real permittivity of TTM near its Curie temperature, shown in Figure 2.10.

\footnotetext{
${ }^{9}$ The reflection coefficient $r(t)$ reduced significantly, indicating excellent microwave absorption near the Curie temperature.

${ }^{10} \mathrm{Use} \approx 20 \mathrm{~cm}^{3} / \mathrm{s}$ to clear transmission line every minute.

${ }^{11}$ As the pyrometer is not controlled through LabVIEW, the acquisition software (Compact Connect) is activated 10 seconds after the LabVIEW software is activated.
} 


\section{Chapter 5}

\section{Results}

This chapter presents the results of experiments performed following the procedure described in Chapter 4. Following this, the first full transient model of the heated ironsand in the cavity is simulated. Then, an investigation in material property (input magnetron power, TTM packing density and BN thermal conductivity) variation is presented. The optimal combination of variations of these parameters is found to result in the best possible agreement with experimental observation. The optimal three-stub tuner protrusion depths are then modelled at $T_{\text {room }}$ and $T_{\text {steady }}$, and the latter is tested against experiment.

\subsection{Experimental results}

The result of an experiment with a small load (20 g) of TTM ironsand in a small BN tube is presented. This measurement provides experimental data, which is then used to validate modelling results. All stubs of the three-stub tuner were fully extracted from the waveguide for this experiment. For this experiment the small bored BN rod was centred in the microwave cavity, filled with TTM ironsand, and heated for 10 minutes under the conditions as set out by the experimental procedure.

The temperature at the surface of the $\mathrm{BN}$ tube in the centre of the cavity is defined as $T(t)$. This was optically measured by CTLaserM2 single colour pyrometer after the temperature measurement was calibrated with QKTRD-4485 two-colour pyrometer. The temperature at the surface of the BN tube, $250 \mathrm{~mm}$ from the centre of the cavity (at the height at which the $\mathrm{BC}$ waveguide termi- 
nates) is defined as $T_{B C}(t)$. This was measured by wrapping the thermocouple around the $\mathrm{BN}$ tube at that height, then packing the ends of the $\mathrm{BC}$ tubes with kaowool. The reflection coefficient is measured via the RF-DC detectors connected to the dual-directional coupler, defined as $P_{b w d}(t) / P_{f w d}(t)=r(t)$. The results of those measurements, are shown as Figure 5.1.
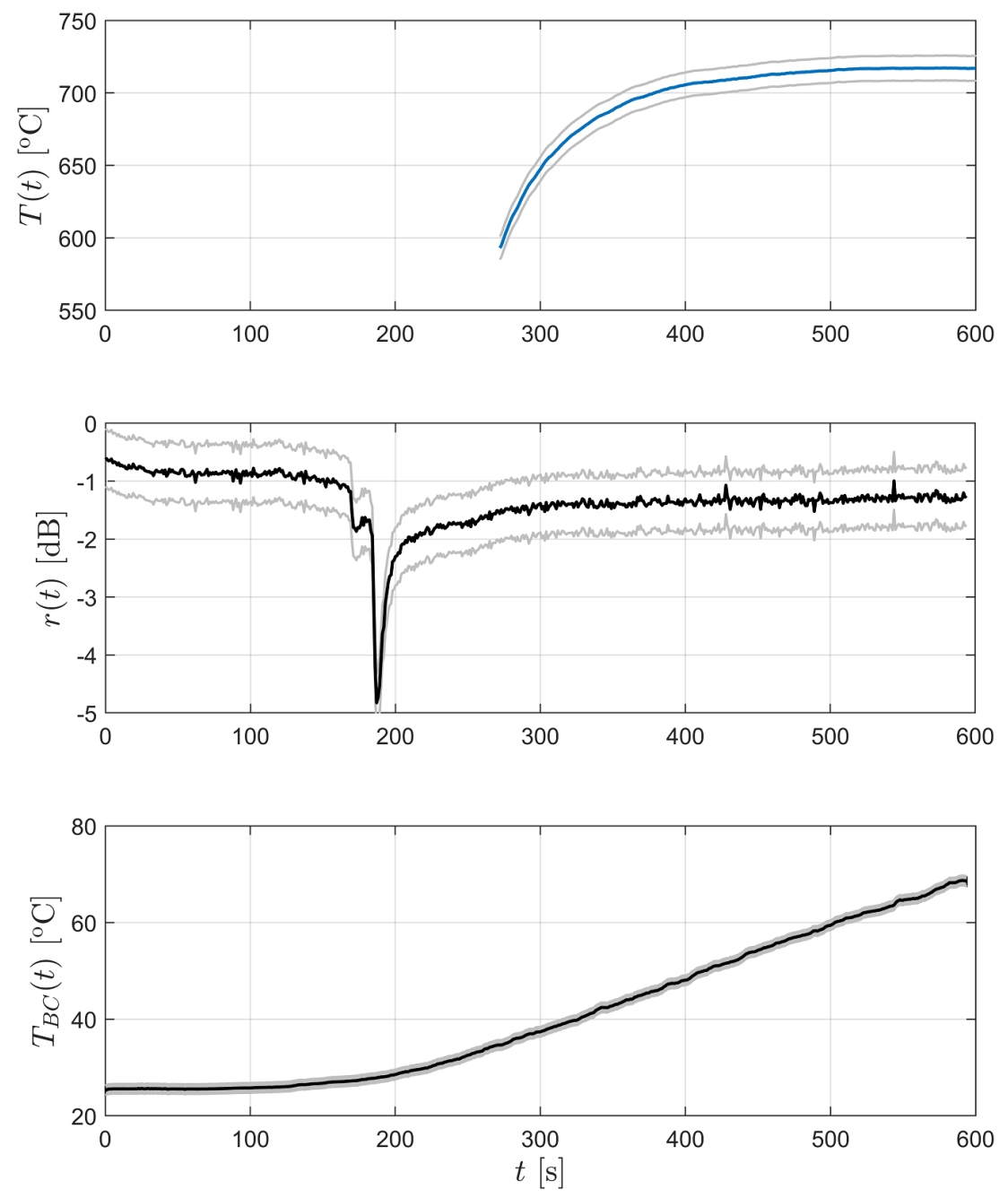

Figure 5.1: Experimental results for the time evolution of the BN tube temperature $T(t)$, cavity reflection coefficient $r(t)$ and $T_{B C}(t)$. A characteristic absorption peak occurs around $t \equiv t_{r e s}=187 \mathrm{~s}$, indicating a sharp change of electromagnetic properties of the ironsand around its Curie temperature. The grey lines represent the standard error of those respective measurements, calculated by error propagation.

A distinct reflection coefficient profile was observed, at $t_{\text {res }}=187 \mathrm{~s}$ where the 


\begin{tabular}{||c|c||}
\hline Parameter & Measured value \\
\hline \hline$r\left(T_{\text {room }}\right)$ & $-1.0 \mathrm{~dB}$ \\
\hline$T_{\text {steady }}$ & $716^{\circ} \mathrm{C}$ \\
\hline$r\left(T_{\text {steady }}\right)$ & $-1.3 \mathrm{~dB}$ \\
\hline$t_{\text {res }}$ & $187 \mathrm{~s}$ \\
\hline$r\left(t_{\text {res }}\right)$ & $-4.7 \mathrm{~dB}$ \\
\hline
\end{tabular}

Table 5.1: Table of key measured data from Figure 5.1.

microwave reflection reaches a minimum that is approximately $-4.7 \mathrm{~dB}$ from an initial reflection amount of $-1.0 \mathrm{~dB}$. The 'sharpness' of $r\left(t_{r e s}\right)$ indicates that the bulk of the ironsand uniformly transitions through $T_{C}$ at $t_{r e s}$. Following $t_{r e s}$, the temperature in the ironsand increases above the minimum temperature threshold of the pyrometer measurement $\left(600^{\circ} \mathrm{C}\right)$, and continues to rise until the temperature appears to converge to $716^{\circ} \mathrm{C}$ over the 600 seconds. A key measurement is that the temperature of the $\mathrm{BN}$ in the cavity rises to over $700{ }^{\circ} \mathrm{C}$ after the onset of high absorption produced by $t_{r e s}$, shown as the top plot in Figure 5.1. This indicates that a significant heating step occurs at time $t_{r e s}$, and that resulting sudden increase in heat energy in the system diffuses throughout the rest of the experiment. It is observed that $T_{B C}(t)$ begins to rise above $T_{\text {room }}$ shortly after $t_{\text {res }}$. The reflected power steadily increases over this time, indicating a reduction in absorption with increasing temperature under these experimental conditions. A steady-state temperature is reached in the furnace when the power losses match the microwave absorption rate. The temperature at the end of the $\mathrm{BN} \operatorname{rod} T_{B C}(t)$ is also seen to increase constantly over time after $t=200 \mathrm{~s}$, as heat is conducted along the BN tube. The results of this measurement are tabulated in Table 5.1.

Repeated experimentation resulted in similar reflection coefficient-time and temperature-time curves as Figure 5.1. After each experiment, the ironsand was removed from the BN tube in the cavity, and replaced with new ironsand. This was done as it was found that ironsand which was repeatedly used in the cavity became 'stuck' to the inner walls of the BN tube, and thereafter presented a different transient heating profile. At low temperatures the reflection coefficient is $-1.0 \mathrm{~dB}$, and at high temperatures the reflection coefficient is $-1.3 \mathrm{~dB}$. The high frequency signal noise observed in the reflected power signal was likely due to slight instability of the magnetron's output power during operation. A key 
issue observed from this experimental data is that- the forward power delivered by the magnetron was found to reduce noticeably during the experimental run. This significantly affected the heating rate of TTM ironsand in the cavity, and implies that the output power of the magnetron dropped over the course of the experiment.

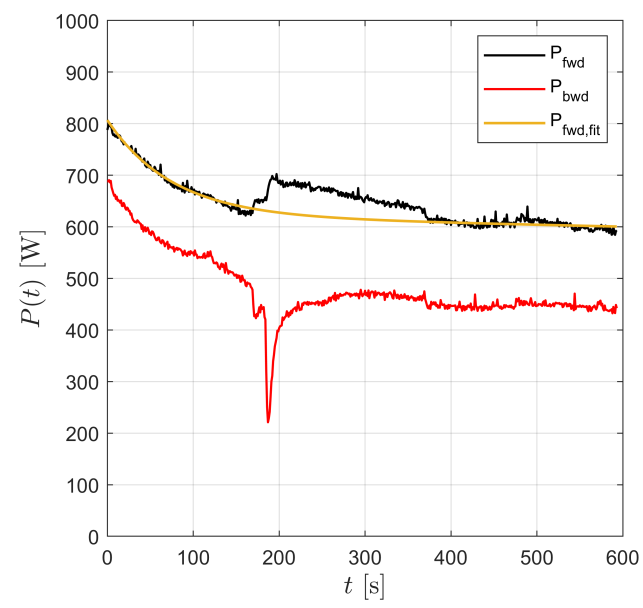

Figure 5.2: Transient evolution of transmitted and reflected power supplied to and from the cavity during an ironsand heating run. This was calculated via the power calibration done in Figure 4.3.1, in Section 4.3.3. The input power reduces from $800 \mathrm{~W}$ to $600 \mathrm{~W}$ over $600 \mathrm{~s}$. This is from the same experimental run as Figure 5.1. The yellow line is a best fit curve of the black input power curve, shown in (Eq. 5.1.1).

One possible cause of this reducing forward power might be that microwave energy was deposited in the magnetron, causing it to overheat as the run progressed. Water cooling of the magnetron was used to minimise the heating of its magnets. However, the magnetron is after the terminator in terms of the order of water-cooled components. The heating power transferred to the load depends on the impedance matching of the magnetron to that load. As the impedance of the load changes, the reflected and absorbed power also changes, and could clearly be observed at around $t_{r e s}$ in Figure 5.2. For implementation of this transient input power decay in the computational model, a smooth analytic curve was required. The forward power appeared to exponentially decay to $\approx 600 \mathrm{~W}$ through the heating run. MATLAB's inbuilt Curve Fitting tool was used to calculate this analytic curve. The forward power measurements between $t=168$ and $t=382 \mathrm{~s}$ were not included in this curve fitting model. A 
linear combination of exponential decay curves $a e^{-b t}+c e^{-d t}$ are used for the curve fitting to best fit the experimental $P_{f w d}$ measurement.

The fitting curve that was used to best match the black curve in Figure 5.2 was:

$$
P_{f w d}=185 e^{-1.33 \times 10^{-2} t}+622 e^{-5.93 \times 10^{-5} t}
$$

which yielded a residual of $R^{2}=0.98$. This completed the experimental aspect of this work. The most robust experimental measurements are the temperature data, as the power measurements have much greater experimental error. In addition to the greater experimental error of those measurements, the calibrations that were made were extrapolated beyond the actual calibration range. This adversely affected the reflection coefficient magnitude measurements.

\subsection{Microwave furnace model investigation}

This section details the modelling process developed and used to describe the thermal and electrical properties of TTM ironsand during microwave heating. The results of changing various properties of the model are highly coupled, so changing one model parameter can have multiple effects on the result of a computational model. Initially an approach was attempted which focused on solving the initial behaviour at room temperature, then at high temperature and finally the transient characteristics of the furnace. However, it was found that this separation of regimes was not a suitable way to model the furnace dynamics, as it was possible for a large range of material property variation combinations to yield the same result.

Modelling a microwave furnace begins with the use of literature values of the relevant materials. Literature values for the properties of each material used in this work were given in Chapter 2. In the case of the ironsand, literature values of magnetite were used as a starting point as this is a highly similar material to the ironsand, and has known electromagnetic properties at $2.45 \mathrm{GHz}$. The input microwave power is not constant over time, so transient heating was modelled with decaying input power (see discussion of Fig. 5.2 above). For modelling steady-state systems (Eq. 5.1.1) is evaluated for $t=600$. This results in a 'steady-state' forward power of $P_{f w d}=600 \mathrm{~W}$, used for modelling steady-state systems. For modelling transient systems, the decaying input power (Eq. 5.1.1) 
was used.

Once the transient heating had been acceptably modelled without using stub protrusions, the optimal depths of the stubs were then predicted via stub depth sweeps in the model. This predicted the optimised stub protrusion lengths for the computational model, and the effects on heating performance of the furnace thereof. The stub positions at high temperatures were then qualitatively compared to experimental results.

\subsection{Modelling results}

The purpose of this section is to detail the process behind all modifications made to the COMSOL model to better match measurement results. The initial model result, Figure 5.1 is compared to the result of the computational model, Figure 5.4. After this, the effect of varying several different properties (magnetron input power, magnetite packing density and $\mathrm{BN}$ thermal conductivity) are examined, in order to determine how best to improve agreement between model and experiment.

A thin $\mathrm{BN}$ rod with a diameter of $1.5 \mathrm{~cm}$ was placed into the furnace, from the end of one $\mathrm{BC}$ waveguide to the end of the other. In the real experiment, this was made from two separate $\mathrm{BN}$ rods. The rod from the bottom has a length of $30 \mathrm{~cm}$, so it protrudes from the end of the lower $\mathrm{BC}$ waveguide to the top of the cavity, which can be seen in Figure 4.10. In this rod, a $1 \mathrm{~cm}$ diameter hole was drilled $10 \mathrm{~cm}$ deep into the end of the $\mathrm{BN}$ rod that is placed into the cavity, seen in Figure 2.6. This hole was then filled with TTM ironsand. The COMSOL model was made to imitate this experimental setup as closely as possible. In this model, air flow was neglected. In the experimental setup of this work, convective heat losses were reduced through plugging the $\mathrm{BC}$ ends to restrict gas flow.

Two optical access holes are included in the model geometry, along with matching holes in the AL-25/1700 fibreboard thermal insulation. The threestub tuner was included for room temperature and steady state temperature optimisation of the reflection coefficient, but would not be implemented until later on in modelling. The spacing from the centre of the cavity to the centre of the first stub of the three-stub tuner was $420 \mathrm{~mm}$ in both the experiment and model. The dimensions of the tuners were discussed in Section 4.1, describing 


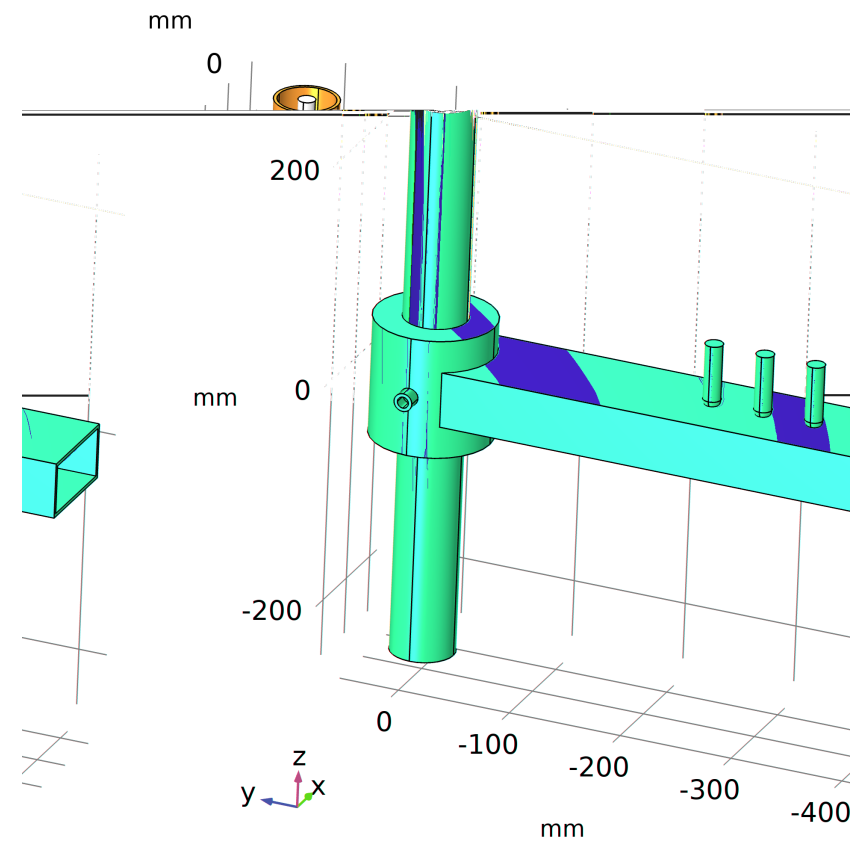

Figure 5.3: An external view of the model geometry used to model heating of TTM contained within the 'thin BN tube'. The BC waveguides, white BN 'tube', optical access holes and three-stub tuner are included to best mirror the experimental setup.

the three-stub tuner in detail. The final model used for subsequent modelling is shown in Figure 5.3.

\subsubsection{Initial model results}

An initial simulation was performed of transient heating over $600 \mathrm{~s}$ and is shown in Figure 5.4. Free time stepping was employed to minimise computation time, governed by the BDF ( backward differentiation formula) method [99] with a maximum order of 4 . The electromagnetic solver used in all subsequent models was GMRES. The thermodynamic solver used in all subsequent models was PARDISO. The predicted reflection coefficient $r$, surface spot temperature $T$ and temperature near end of $\mathrm{BC}$ waveguide $T_{B C}$ are plotted against time for 600 seconds. The transient input power in the model ${ }^{1}$ was set according to (Eq. 5.1.1). The room temperature was set to $26^{\circ} \mathrm{C}$ to best match experimental conditions in the laboratory. The properties of materials used in this model are the exact temperature-dependent values taken from Section 2.5 in Figures 2.7

\footnotetext{
${ }^{1}$ Set as a $\mathrm{TE}_{10}$ mode input port of $2.45 \mathrm{GHz}$ microwave power.
} 
and 2.10. The material properties for use in modelling are linearly interpolated between data points, and linearly extrapolated beyond their measured ranges and stored in 'look-up tables' for COMSOL to retrieve the specific material property any temperature. No other material parameters have been further adjusted.
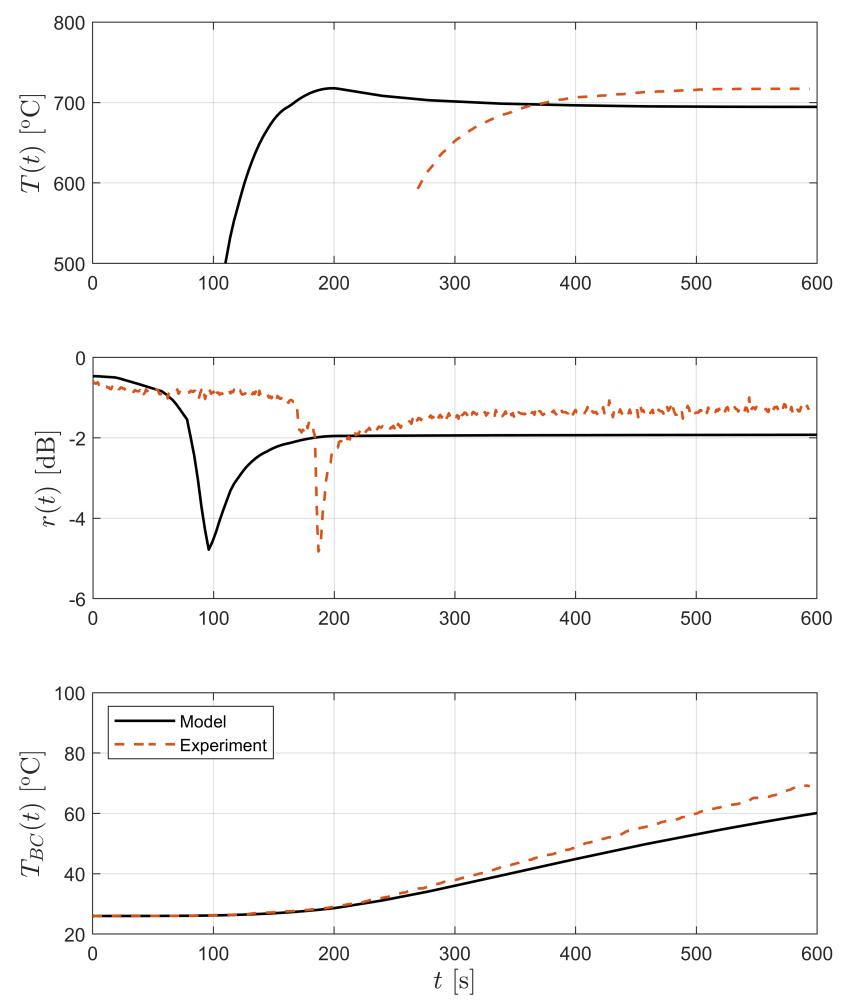

Figure 5.4: Simulated transient plots for microwave heating of ironsand in the experimental furnace. A clear resonance occurs at $t=96 \mathrm{~s}$, which temporarily delivers a greatly increased rate of heating.

A table of key results of this simulation (Table 5.2) are useful for comparison with the experimental results in Table 5.1.

Comparing the distinct differences between model and experiment, the model showed a lower rate of microwave absorption, however a faster rate of heating. There are clear magnitude $(r(t))$ and temporal $t_{r e s}$ discrepancies between this initial model and experimental results. The general 'profile' of the curve was however present, implying this was an acceptable starting point for modelling the dynamics of the microwave furnace. The properties of the model around $t_{r e s}$ was then the most interesting aspect of the entire experiment. From Figure 2.10 it is observed that the real permittivity of TTM increases when 


\begin{tabular}{||c|c||}
\hline Parameter & Modelled value \\
\hline \hline$r\left(T_{\text {room }}\right)$ & $-0.47 \mathrm{~dB}$ \\
\hline$T_{\text {steady }}$ & $694^{\circ} \mathrm{C}$ \\
\hline$r\left(T_{\text {steady }}\right)$ & $-1.92 \mathrm{~dB}$ \\
\hline$t_{\text {res }}$ & $96 \mathrm{~s}$ \\
\hline$r\left(t_{\text {res }}\right)$ & $-4.8 \mathrm{~dB}$ \\
\hline
\end{tabular}

Table 5.2: Table of key initial modelling results taken from Figure 5.4.

$T$ is close to $T_{C}$. This same profile is observed in the model as when TTM is heated in experiment. The profile of the electromagnetic field energy density, $U_{E M}$, during its transition through $t_{r e s}$ is shown in Figure 5.5. Three instants are chosen, each $30 \mathrm{~s}$ apart, to demonstrate the changing energy density field profile throughout the resonance.
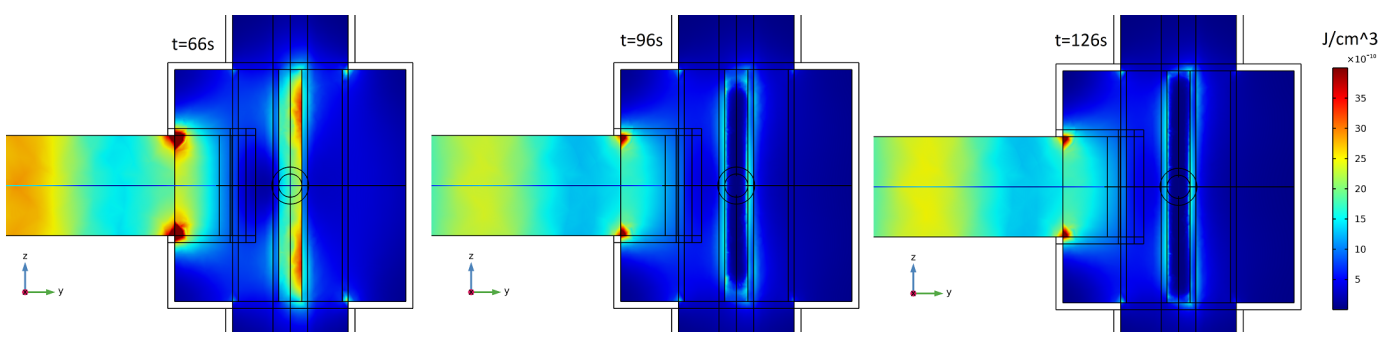

Figure 5.5: Side-on view of $U_{E M}$ (Eq. 2.1.5) in cavity during model run of Figure 5.4 at various instants during transient simulation of the model. Left; $t=66 \mathrm{~s}$ : middle: $t=96 \mathrm{~s}$, right: $t=126 \mathrm{~s}$. The ironsand is located in the centre of the cavity.

In Figure 5.5, $t=66 \mathrm{~s}$ the plotted electromagnetic field energy density shows high field densities in two locations along the central axis of the ironsand 'rod'. This high field density results in excellent ironsand heating at those locations. This eventually brings the temperature at those points to the Curie temperature, at $t=96 \mathrm{~s}$. Due to the significantly increasing conductivity of the modelled ironsand, this 'ejects' the electric field to the surface of the modelled ironsand $^{2}$. Beyond $T_{C}$, electrical losses dominate, resulting in the low (dark blue) energy density in the ironsand in the cavity. This effect could be seen

\footnotetext{
${ }^{2}$ As the microwave penetration into the ironsand reduces due to the skin effect at high conductivities [36].
} 
at both $t=96 \mathrm{~s}$ and $t=126 \mathrm{~s}$. Beyond $t=126 \mathrm{~s}$, conductive surface losses dominate the microwave absorption of the ironsand in the cavity.

\subsubsection{Model refinements}

Since the experiment has three measured curves, a combination of three parameters are used to best fit the behaviour of the model to experimental result. The important features to best fit the model to are; $t_{r e s}, T_{\text {steady }}$ and $T_{B C}(t)$. The sharpness of the absorption peak at $t_{\text {res }}$ is a key feature of the experimental results. The current discrepancy in width of the absorption peak is due to the linear extrapolation of $\mu_{r}(T)$ from Figure 2.10. The distinct absorption peak is a result of the near-uniform transition of most of the ironsand through its Curie temperature. Parameters of the system that are varied must maintain the distinct reflection coefficient profile. Three factors are defined:

- Input power with factor $\alpha \leq 1$. This term determines the magnitude of the power incident in the cavity through scalar multiplication with the measured power from (Eq. 5.1.1). Reducing this term will delay the modelled $t_{\text {res }}$, and reduce $T_{\text {steady }}$.

- Ironsand packing factor with $\beta \leq 1$. This factor controls the magnitude of the permittivity $\varepsilon_{r}^{\prime}-i \varepsilon_{r}^{\prime \prime}$, permeability $\mu_{r}^{\prime}-i \mu_{r}^{\prime \prime}$, heat capacity $c_{p}$ and thermal conductivity $k_{t}$ of the ironsand. Reducing this term will again delay the modelled $t_{\text {res }}$, but increase $T_{\text {steady }}$ due to a lowered thermal mass of the ironsand in the cavity.

- BN thermal conductivity with factor $\gamma \geq 1$. This term is a composite term modelling both conductive and convective losses in the model. Increasing this term would slightly delay $t_{\text {res }}$, and slightly lower $T_{\text {steady }}$. Increasing $\gamma$ increases the rate of heat flow through $T_{B C}$.

These factors $\alpha, \beta$ and $\gamma$ were chosen as they all represented a unique aspect of the furnace model. For the case of the input power, the exact magnitude of the energy deposited into the cavity had some systematic errors during calibration due to the uncertainty in water flow rate through the water cooling. The case for the ironsand packing factor is that ironsand is a distinctly different material and will have properties differing from the literature values of magnetite, taken from Figure 2.10. This may explain the reduction of the 
magnitudes of the $\varepsilon_{r}, \mu_{r}, k_{t}$ and $c_{p}$ material parameters for the ironsand, when compared to similar magnetite powders. Furthermore, the thermal conductivity of BN was increased in the model to accommodate for the other thermal losses in the system which were not included in the COMSOL model. The most significant type of losses that are not considered are convective heat losses due to the flowing $\mathrm{N}_{2}$ gas. A variety of independent steady-state models are evaluated for specific values near literature values of each function; $\alpha, \beta$ and $\gamma$. Firstly, a parameter sweep for the input power $\alpha$ is done, comparing the effects of each of these values on $r\left(T_{\text {room })}, r\left(T_{\text {steady }}\right)\right.$ and $T_{\text {steady }}$. At this stage, $t_{\text {res }}$ can not be evaluated ${ }^{3}$. The results of the variation of factor $\alpha$ are tabulated in Table 5.3.

\begin{tabular}{||c|c|c||}
\hline Cavity input power $\alpha P_{f w d}$ & $r\left(T_{\text {steady }}\right)$ & $T_{\text {steady }}$ \\
\hline \hline$\alpha=0.6$ & $-2.58 \mathrm{~dB}$ & $614^{\circ} \mathrm{C}$ \\
\hline$\alpha=0.7$ & $-2.36 \mathrm{~dB}$ & $637^{\circ} \mathrm{C}$ \\
\hline$\alpha=0.8$ & $-2.18 \mathrm{~dB}$ & $657^{\circ} \mathrm{C}$ \\
\hline$\alpha=0.9$ & $-2.04 \mathrm{~dB}$ & $676^{\circ} \mathrm{C}$ \\
\hline$\alpha=1$ & $-1.92 \mathrm{~dB}$ & $694^{\circ} \mathrm{C}$ \\
\hline
\end{tabular}

Table 5.3: Table of steady-state temperature and reflection coefficients upon variation of input power $P_{f w d}$ by factor $\alpha$. The original values for $T_{\text {steady }}$ and $r\left(T_{\text {steady }}\right)$ as from Table 5.2 are retrieved when $\alpha=1$. Note the omission of $r\left(T_{\text {room }}\right)$, this is due to the room temperature reflection not depending on the magnitude of the input power.

Table 5.3, which shows that the steady-state temperature is dependent on the input power. Reducing $\alpha$ was predicted to delay $t_{r e s}$ as less power is absorbed over a fixed time $t$ if $P_{f w d}$ is reduced. The resulting steady-state temperature $T_{\text {steady }}$ also reduced. The next factor that is tested is the relative packing factor of magnetite, $\beta$, and the results of those simulations are shown in Table 5.4 .

In Table 5.4, as the packing density of the ironsand was reduced, its steadystate temperature $T_{\text {steady }}$ is increased due to the reduced thermal mass of the material. In addition to this, the energy absorbed by the ironsand was reduced

\footnotetext{
${ }^{3}$ This is because the modelling method used was a steady-state method, which only simulates the $\partial_{t} T$ temperature field.
} 


\begin{tabular}{||c|c|c|c||}
\hline Ironsand packing factor $\beta$ & $r\left(T_{\text {room }}\right)$ & $r\left(T_{\text {steady }}\right)$ & $T_{\text {steady }}$ \\
\hline \hline$\beta=0.5$ & $-0.23 \mathrm{~dB}$ & $-2.28 \mathrm{~dB}$ & $763^{\circ} \mathrm{C}$ \\
\hline$\beta=0.6$ & $-0.27 \mathrm{~dB}$ & $-2.17 \mathrm{~dB}$ & $744^{\circ} \mathrm{C}$ \\
\hline$\beta=0.7$ & $-0.31 \mathrm{~dB}$ & $-2.08 \mathrm{~dB}$ & $728^{\circ} \mathrm{C}$ \\
\hline$\beta=0.8$ & $-0.36 \mathrm{~dB}$ & $-2.01 \mathrm{~dB}$ & $714^{\circ} \mathrm{C}$ \\
\hline$\beta=0.9$ & $-0.41 \mathrm{~dB}$ & $-1.96 \mathrm{~dB}$ & $703^{\circ} \mathrm{C}$ \\
\hline$\beta=1$ & $-0.47 \mathrm{~dB}$ & $-1.92 \mathrm{~dB}$ & $694^{\circ} \mathrm{C}$ \\
\hline
\end{tabular}

Table 5.4: Table of steady-state temperature and reflection coefficients upon variation of ironsand packing fraction by factor $\beta$. The original values for $T_{\text {steady }}$ and $r\left(T_{\text {steady }}\right)$ as from Table 5.2 are retrieved when $\beta=1$.

at low temperatures for any packing factor $\beta<1$, leading to a delay in $t_{\text {res }}$. Reducing $\beta$ delays $t_{r e s}$, and increases $T_{\text {steady }}$, making it a distinct and useful parameter to consider. The next factor to model was $\gamma$, the $\mathrm{BN}$ thermal conductivity, the results of which are displayed in Table 5.5.

\begin{tabular}{||c|c|c||}
\hline BN thermal conductivity $\gamma k_{B}$ & $r\left(T_{\text {steady }}\right)$ & $T_{\text {steady }}$ \\
\hline \hline$\gamma=1$ & $-1.92 \mathrm{~dB}$ & $694^{\circ} \mathrm{C}$ \\
\hline$\gamma=1.25$ & $-1.95 \mathrm{~dB}$ & $690^{\circ} \mathrm{C}$ \\
\hline$\gamma=1.5$ & $-1.99 \mathrm{~dB}$ & $684^{\circ} \mathrm{C}$ \\
\hline$\gamma=1.75$ & $-2.01 \mathrm{~dB}$ & $679^{\circ} \mathrm{C}$ \\
\hline$\gamma=2$ & $-2.04 \mathrm{~dB}$ & $675^{\circ} \mathrm{C}$ \\
\hline
\end{tabular}

Table 5.5: Table of steady-state temperature and reflection coefficients upon variation of BN thermal conductivity by factor $\gamma$. The original values for $T_{\text {steady }}$ and $r\left(T_{\text {steady }}\right)$ as from Table 5.2 are retrieved when $\gamma=1$. Note the omission of $r\left(T_{\text {room }}\right)$; this is due to the room temperature reflection not depending on the thermal conductivity of BN.

From Table 5.5, every increase of $25 \%$ of the thermal conductivity of $\mathrm{BN}$, the steady-state temperature reduced by $\approx 5^{\circ} \mathrm{C}$. Tables $5.3,5.4$ and 5.5 demonstrate that the different measured factors of the cavity from Table 5.1 are highly coupled. A unique combination of both $\alpha$ and $\beta$ result in $t_{\text {res }}$ and $T_{\text {steady }}$ to better match experimental observation. In addition, an increased $\gamma$ would also slightly delay $t_{\text {res }}$. The prefactors $\alpha$ and $\beta$ were set to 0.9 and 0.6 respectively as an initial guess, to approximately match $t_{r e s}$ and $T_{\text {steady }}$ 
in experiment. Deviations from this combination of prefactors would result in worse agreement with experimental results. The next step was to check if that guess of parameter combinations was correct. The desired result of that transient simulation at this point was: a slight underestimation for $t_{\text {res }}$ and an overestimation for $T_{\text {steady }}$ when compared to the experimental result from Figure 5.1. This would allow for some tolerance with an increased $\gamma$, which would slightly delay $t_{\text {res }}$ and reduce $T_{\text {steady }}$ as in Table 5.5, while heavily improving agreement with $T_{B C}(t)$.
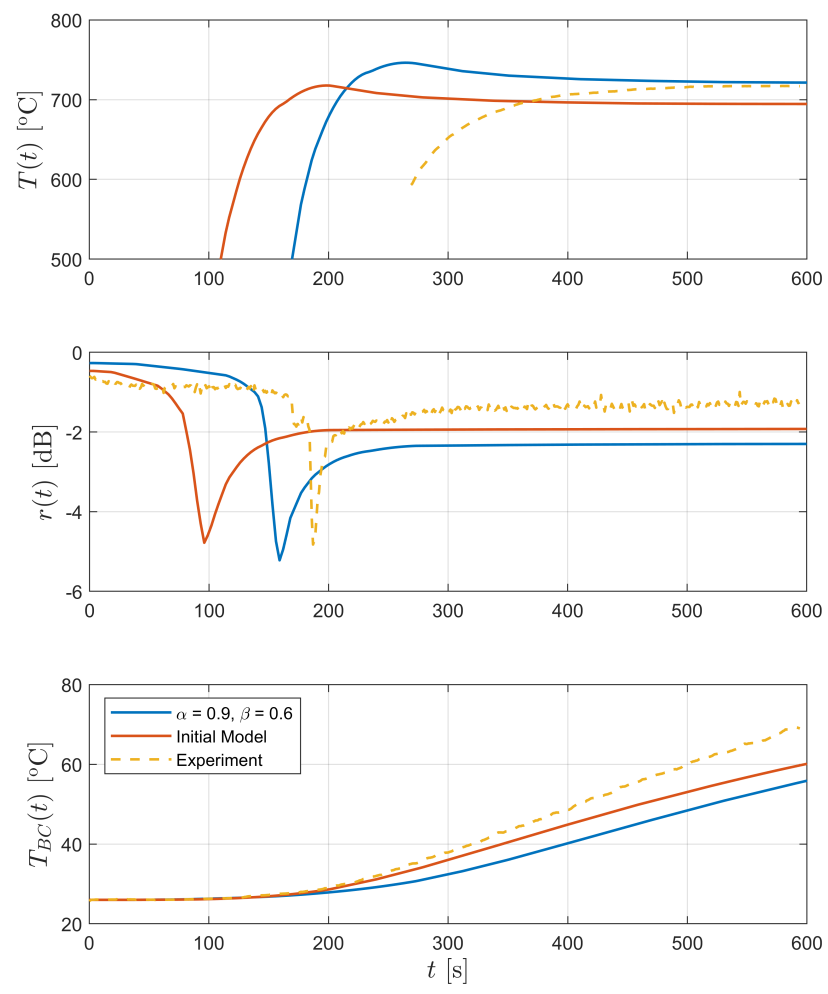

Figure 5.6: Simulated transient plots for microwave heating of ironsand in the experimental furnace. The varied parameters $\alpha=0.9$ and $\beta=0.6$ result in improved agreement between model and experiment. The blue line is the updated model, the orange is the original model from Figure 5.4 and the yellow dashed line is experimental result.

Figure 5.6 shows an improved agreement between model (blue) and experiment (yellow, dashed) from the original model (orange) for $T(t), r(t)$ and $t_{\text {res }}$. In Figure $5.6, t_{r e s}$ leads the experimental $t_{r e s}$, and the steady-state temperature also exceeds experimental result. The under-damped temperature profile in both modelled curves in Figure 5.6 indicates that too much heat energy is 
transferred by the modelled ironsand to the BN tube. The discrepancy between model and experiment could be due to more complex heat energy transfer dynamics between the ironsand powder, and the BN tube. This would delay the diffusion of heat energy from the TTM to the BN crucible, as the TTM ironsand passes as a bulk through the Curie temperature $\left(T_{C}=430^{\circ} \mathrm{C}\right)$.

However, the transient heating $T_{B C}(t)$ agreement with experiment was worsened. The BN thermal conductivity factor was then varied to $\gamma=1.25$, otherwise under the same conditions $(\alpha=0.9, \beta=0.6)$ as in Figure 5.6. This would further delay $t_{\text {res }}$, reduce $T_{\text {steady }}$ and improve agreement between model and experiment for $T_{B C}(t)$. It was then found; as shown in Figure 5.7, that this combination of $\alpha=0.9, \beta=0.6, \gamma=1.25$ was close to the experimental measurement curve. However it was found that $t_{\text {res }}$ was marginally too early, and $T_{B C}(t)$ was slightly underestimated by the model. This was remedied by lowering the packing factor of the ironsand via $\beta=0.55$ to further delay the resonance onset. This also raises the steady-state temperature analogously to Table 5.4. In addition, the BN thermal conductivity was increased with $\gamma=1.3$, to increase the heat flow to the end of the BN tube and to reduce the steady-state temperature, again analogously to Table 5.5.

Figure 5.7 shows good agreement between the modified system parameters $\alpha=0.9, \beta=0.55, \gamma=1.3$ and experiment. The core discrepancies between the 'best-fitted' model (yellow, Figure 5.7) and experiment (dashed purple, Figure 5.7) are as follows:

- $T(t)$ profile shortly after $t_{\text {res }}$. This could have been due to unaccounted thermal energy transfer dynamics between the ironsand grains and walls of the BN tube. This was simulated poorly by COMSOL because the ironsand is modelled in these simulations as a 'bulk' material.

- The magnitudes of $r\left(T_{\text {room }}\right)$ and $r\left(T_{\text {steady }}\right)$. This could have been due to an unreliably extrapolated power calibration.

The model in Figure 5.7 appeared to slightly underestimate the temperature $T_{B C}$ around $t=300 \mathrm{~s}$, and strongly overestimates the rate of heat transfer onto the surface of the centre of the BN tube, resulting in a modelled underdamped heating, which is not the case in the experimental result, where the system appears overdamped. This would indicate that in experiment, more heat energy is transferred into the extremities of the $\mathrm{BN}$ rods. The temperature $T(t)$ rises 

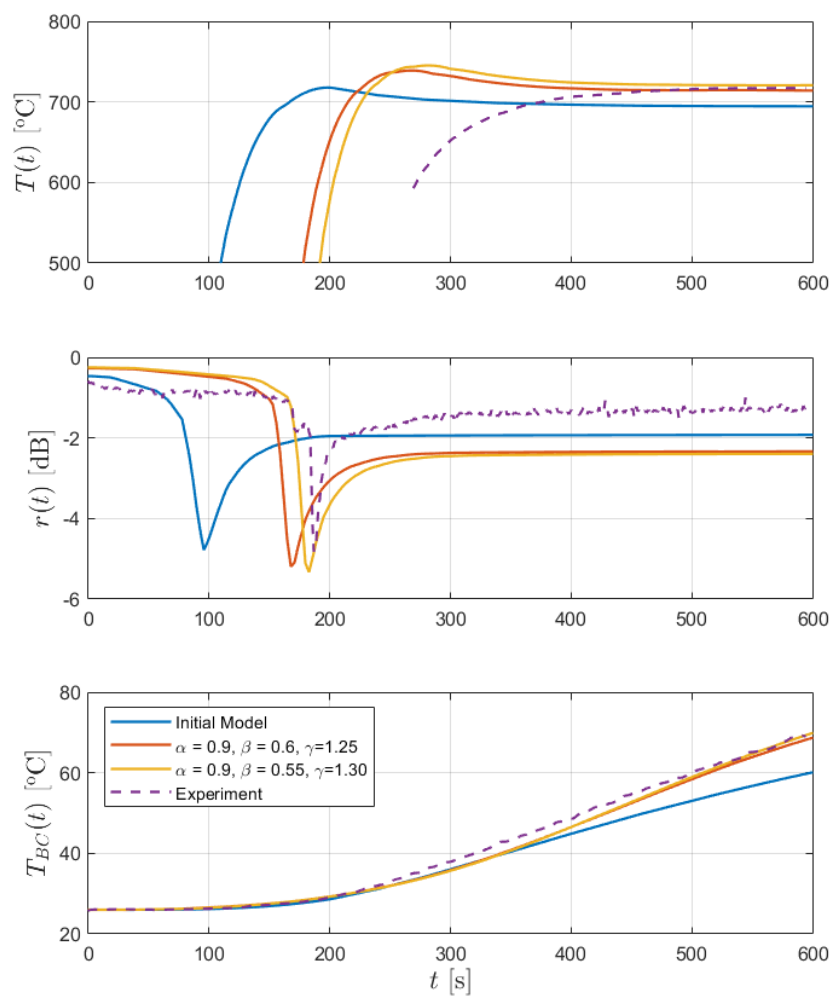

Figure 5.7: Simulated transient plots for microwave heating of ironsand in the experimental furnace. The varied parameters $\alpha, \beta$ and $\gamma$ result in a further improved agreement between model and experiment from Figure 5.6.

far too rapidly near $t_{\text {res }}$, and was suspected to be due to the low thermal contact between the ironsand grains and the drilled-out inner BN rod wall.

To summarise this section: three transient curves were measured in experiment (Figure 5.1), and an initial computational model was produced (Figure 5.4). Then, the model was improved by variation of three parameters, and the experimental results $\left(T_{\text {steady }}, t_{\text {res }}\right.$ and $\left.T_{B C}\right)$ were 'fitted' using those parameters $\alpha, \beta$ and $\gamma$. This resulted in a unique combination of $\alpha, \beta, \gamma$ to result in the best-fit model as shown in Figure 5.7. This demonstrates the limitations of the method used for the model investigation in this work. However, with this computational model, the key aspects (i.e. $\left.T_{\text {steady }}, t_{\text {res }}\right)$ of the heating dynamics of the microwave furnace are accurately modelled. 


\subsection{Modelling the three-stub tuner}

The three-stub tuner gives control of the microwave propagation through the transmission line, and hence reflection and transmission of microwave power into, and out of the microwave cavity. This was observed in multiple experiments, where the highest temperatures within the cavity were produced while the three-stub tuner was actively tuned to minimise reflected power $r$ while at high temperatures. This section aims to determine both the high temperature arrangement of stubs. This would maximise the temperature reached by the ironsand in the cavity. For completeness, this modelling process was also done at room temperature. This indicates the best combination of positions for the stubs to result in greatest initial heating rate at low temperatures. Finally, transient heating curves are computed for both cases over $1000 \mathrm{~s}$. The heating performance of the room temperature optimised stub positions, and the maximum steady-state temperature stub positions are also compared to the original un-tuned model.

To model the effects of of the three-stub tuner, a parameter sweep was done of the stub depths at both room temperature and steady-state temperatures. The method by which the optimised combination of stub protrusions is completed is by optimisation of one stub protrusion depth at a time. For example; to establish the optimised stub positions for the room temperature case, all possible independent stub depths are independently modelled. From this set of models, for the specific stub, the associated stub depth with the greatest reduction in $r\left(T_{\text {room }}\right)$ is chosen. This becomes a fixed stub protrusion depth for the rest of the optimisation process. The second step was to vary the depths of the other two stubs, then to choose the stub (and its associated depth) that resulted again in a further reduction in $r$. That second stub was then fixed for the last step in the optimisation process. The same stub sweep process was done for the last stub, where the final optimised stub depth was established. This process is repeated for the high temperature stub position optimisation. These stub arrangements minimise $r\left(T_{\text {room }}\right)$ and $r\left(T_{\text {steady }}\right)$ respectively.

The maximum stub turns in this modelling stage is 21 turns (to match the limit of turns in the real three-stub tuner), while the step size for the room temperature modelling stage is 1 turn. For the $r\left(T_{\text {steady }}\right)$ optimisation process, a larger step size (3 turns) was used due to the greatly increased computation 
time.

\subsubsection{Low temperature stub optimisation}

Optimising the stubs for low temperatures results in a furnace with an increased heating rate. In the first step all stubs are independently moved into the waveguide to maximise microwave absorption (i.e. to minimise $r\left(T_{\text {room }}\right)$ ).

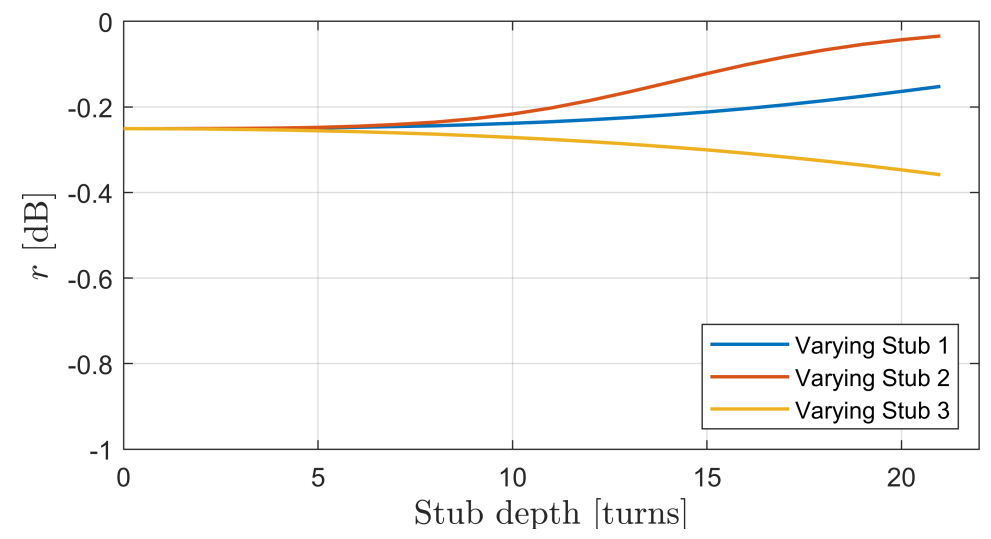

Figure 5.8: Various independent stub protrusions of all three stubs, resulting in a varied reflection coefficient $r\left(T_{\text {room }}\right)$. Moving stub 3 to 21 turns resulted in improved microwave absorption.

Both stub 1 and stub 2 in Figure 5.8 appeared to worsen $r\left(T_{\text {room }}\right)$, but increasing the depth of stub 3 (yellow) to 21 turns showed an increase in microwave absorption. This improved the microwave absorption from $-0.25 \mathrm{~dB}$ without tuning, to $-0.36 \mathrm{~dB}$ after tuning stub 3 to 21 turns. This process was repeated to find the optimised stub positions for stubs 1 and 2, while stub 3 was fixed to 21 turns.

In Figure 5.9, tuning the second stub (orange) to 9 turns resulted in a significant reduction in $r\left(T_{\text {room }}\right)$ from $-0.36 \mathrm{~dB}$ to $-2.19 \mathrm{~dB}$. Tuning the first stub (blue) independently to the second stub did not improve absorption at this stage. The second stub was fixed to a depth of 9 turns. The last simulation tested the optimised position of the first stub, while the second and third stubs were fixed to 9 , and 21 turns respectively.

The optimum depth for stub 1 from Figure 5.10 was 1 turn, and it improved absorption performance marginally over zero turns to $-2.20 \mathrm{~dB}$. Tuning the first stub between 0 and 4 turns showed no significant change in $r\left(T_{\text {room }}\right)$, 


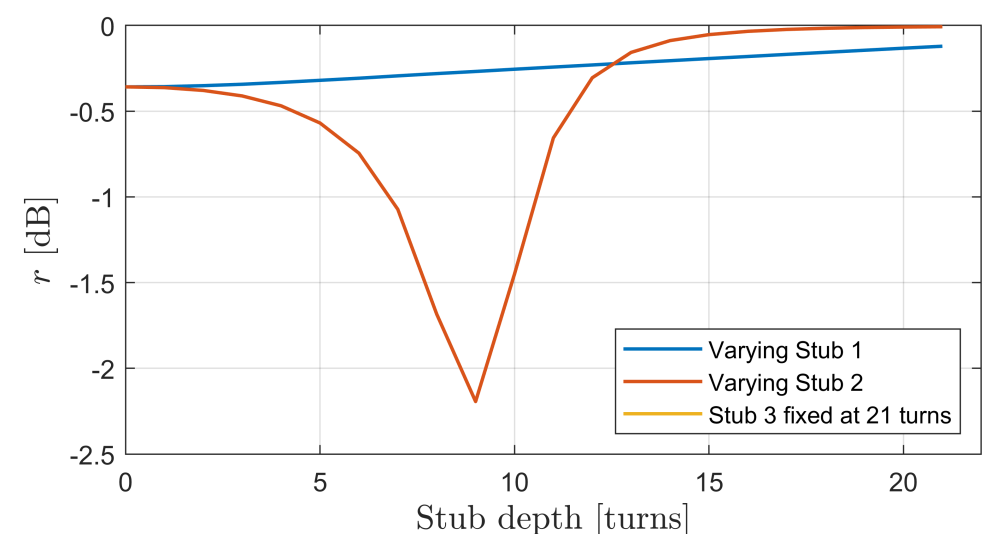

Figure 5.9: Various independent stub protrusions of stub 1 and 2, resulting in a further varied reflection coefficient $r\left(T_{\text {room }}\right)$ depending on stub depths. In these models, the depth of stub 3 was fixed to 21 turns. Moving stub 2 to 9 turns resulted in improved microwave absorption.

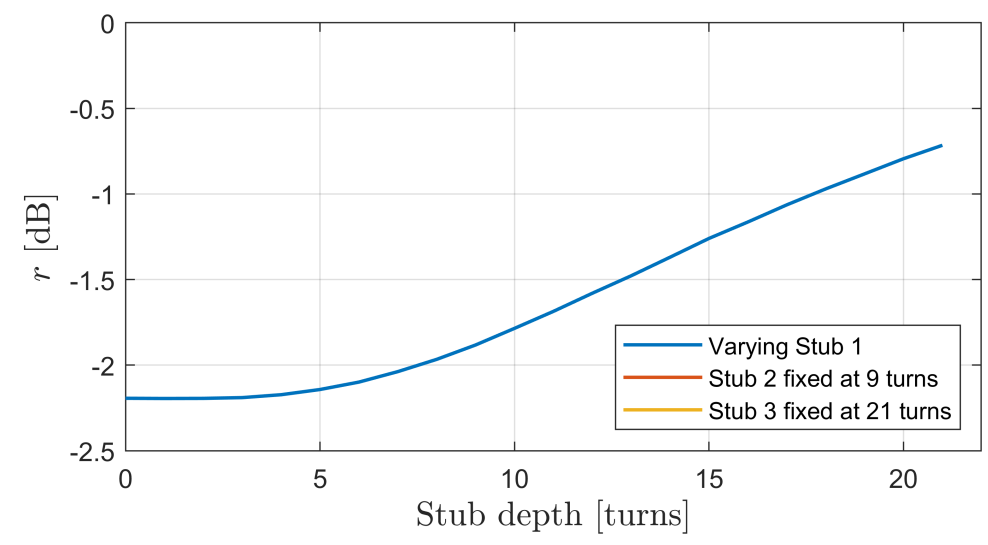

Figure 5.10: Various independent stub protrusions of stub 1, resulting in a worsened reflection coefficient $r\left(T_{\text {room }}\right)$. In this model, the depth of stub 3 was fixed to 21 turns, and the depth of stub 2 was fixed to 9 turns.

observable in Figure 5.10. Optimising the stub tuning arrangement results in a reduction of reflected power of $94 \%$ to $63 \%$, calculated via (Eq. 4.3.4), resulting in a five-fold greater power transfer rate at low temperatures. The resulting stub positions are shown in Figure 5.11. 


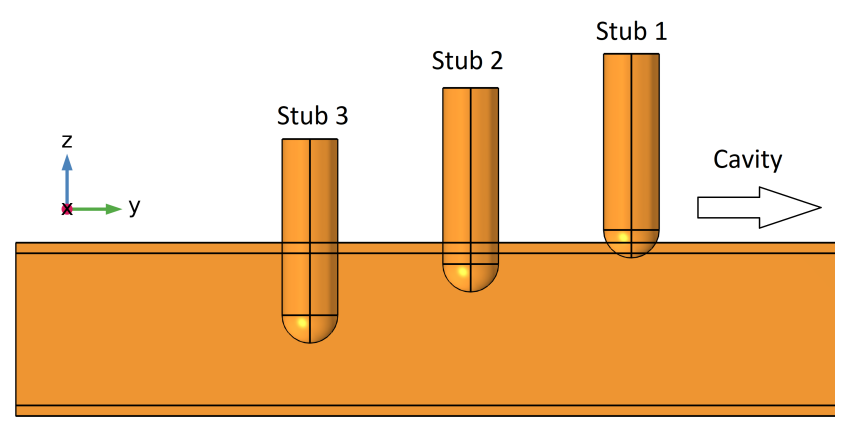

Figure 5.11: Cross section of WR340 waveguide section of Figure 5.3. The tuning stubs are shown in the positions optimised for low temperature heating (as predicted by the modelling in this section) with stub turns of 1,9 and 21 respectively for stubs 1,2 and 3 .

\subsubsection{High temperature stub optimisation.}

As with the low temperature model, the stubs depths were independently tested and iteratively fixed to maximise microwave absorption in steady-state models. This set of models would indicate the optimal stub depths for reaching the highest temperature $T_{\text {steady }}$ possible in the furnace, by minimising the reflected microwave power $r\left(T_{\text {steady }}\right)$. The first of the various depth variations are shown in Figure 5.12.

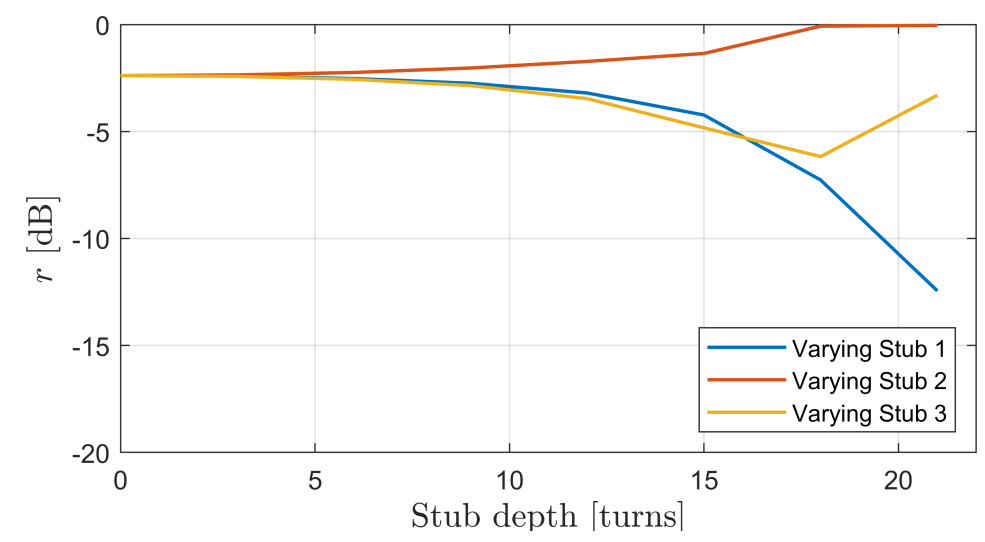

Figure 5.12: Various independent stub protrusions of all three stubs, resulting in a varied reflection coefficient for steady-state models. Moving stub 1 to 21 turns resulted in improved microwave absorption.

Protruding stub 1 again fully into the transmission line with 21 turns 
results in a significant reduction in reflected microwave power from $-2.39 \mathrm{~dB}$ to $-12.45 \mathrm{~dB}$, greatly increasing the power transfer rate to the ironsand, shown in Figure 5.12. For the next models, the first stub is fixed at its maximum turn number (21) while the second and third stub depths are varied independently.

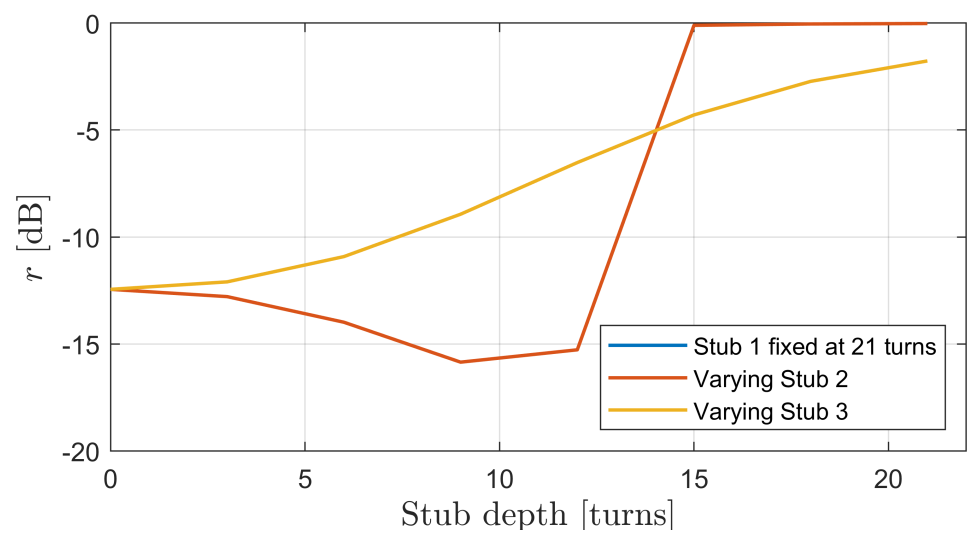

Figure 5.13: Various independent stub protrusions of stubs 2 and 3, resulting in a further varied high temperature reflection coefficient depending on stub depths. In these models, the depth of stub 1 was fixed to 21 turns. Moving stub 2 to 9 turns resulted in improved microwave absorption.

Increasing the depth of stub 9 appeared to worsen microwave absorption, whereas when stub 2 was protruded to a depth of 9 turns, the absorption increased from $-12.45 \mathrm{~dB}$ to $-15.85 \mathrm{~dB}$ as shown in Figure 5.13. Finally, the third stub is protruded into the cavity while stub 1 and stub 2 were fixed at 21 turns, and 9 turns respectively. The result of this protrusion depth sweep is shown in Figure 5.14.

Increasing the protrusion depth of stub 3 while stub 1 and stub 2 were fixed did not reduce $r\left(T_{\text {steady }}\right)$ further than before. Thus, the modelled optimised stub positions to maximise $T_{\text {steady }}$ was 21 turns, 9 turns and 0 turns respectively for stubs 1, 2 and 3. This can be visualised similarly to Figure 5.11 in Figure 5.15 .

The temperature of these associated stub depth combinations was then reviewed. From Figure 5.14, stub depth of stub 3 was plotted against the associated $T_{\text {steady }}$ temperatures shown in Figure 5.16, while stub 1 and 2 were fixed at the depths outlined as shown in Figure 5.15.

Thus, by tuning the depth of the third stub, a variety of steady-state temperatures were achieved in simulation, ranging from $695^{\circ} \mathrm{C}$ to $1034^{\circ} \mathrm{C}$, 


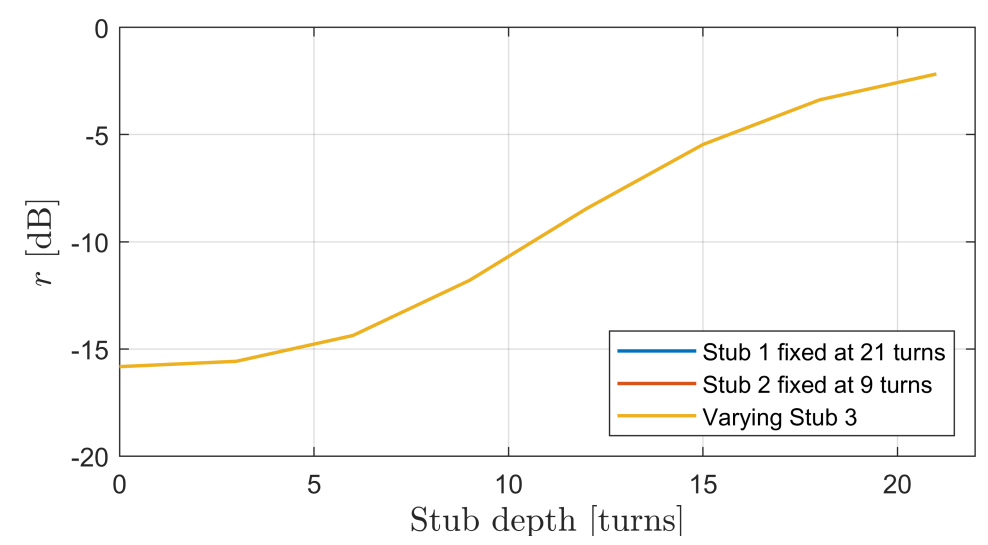

Figure 5.14: Various independent stub protrusions of stub 3, resulting in a further varied reflection coefficient at $r\left(T_{\text {steady }}\right)$. In this model, the depth of stub 1 was fixed to 21 turns, and the depth of stub 2 was fixed to 9 turns.

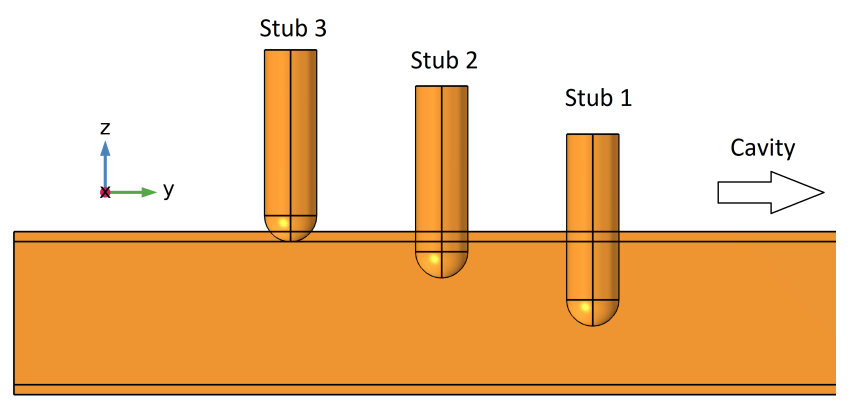

Figure 5.15: Cross section of WR340 waveguide section of Figure 5.3. The tuning stubs are shown in the positions optimised for maximum spot temperature $T_{\text {steady }}$ (as predicted by the modelling in this section) with stub turns of 21,9 and 0 respectively for stubs 1,2 and 3 .

meaning that the stub depth positions could be used as a potential method of temperature control in the microwave furnace. The model prediction of the optimal positions of the stubs was then tested against experimental observation.

The experimental procedure in this test was similar to the default heating method; however once the system was near a steady-state temperature, the stubs were actively tuned to further increase $T_{\text {steady }}$. The optimal combination of stub positions was found via repeated tuning of each stub. The process is as follows: the stub closest to the cavity was tuned to result in maximum absorption; following this, the second stub was tuned after a new steady-state temperature was reached; finally the same is done with the third stub. If at any 


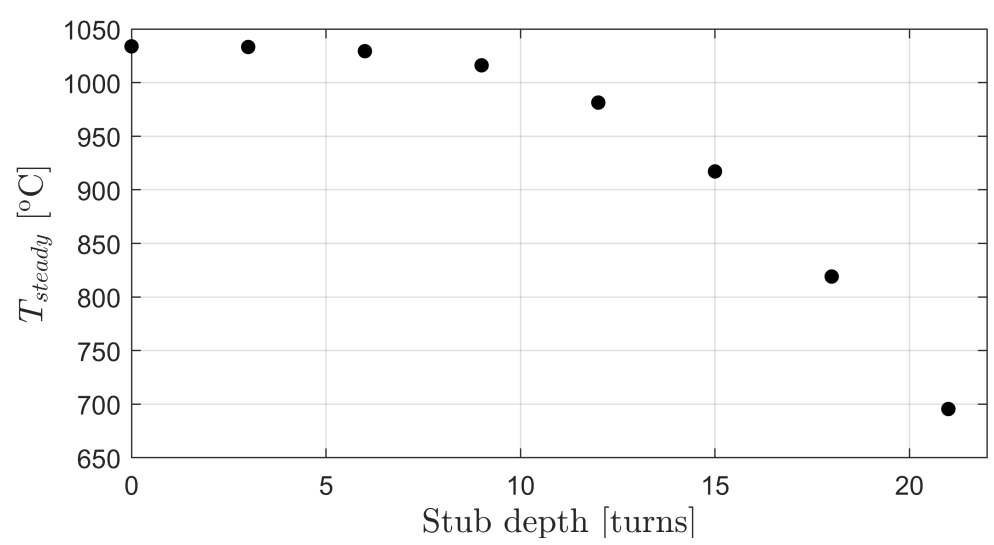

Figure 5.16: Various stub 3 depths and those associated steady-state temperatures. An increased stub depth resulted in worsened microwave absorption, and therefore a reduced $T_{\text {steady }}$. This is the same dataset from the variation of the depth of stub 3 in Figure 5.14.

stage the measured temperature appears to reduce, that stub was reverted to its previous position before that tuning step, 'reverting' the loss in temperature. This process can be seen in Figure 5.17. This process was repeated until moving any stub would result in a lowered steady-state temperature. A peak temperature of $1034{ }^{\circ} \mathrm{C}$ was experimentally observed in Figure 5.17.

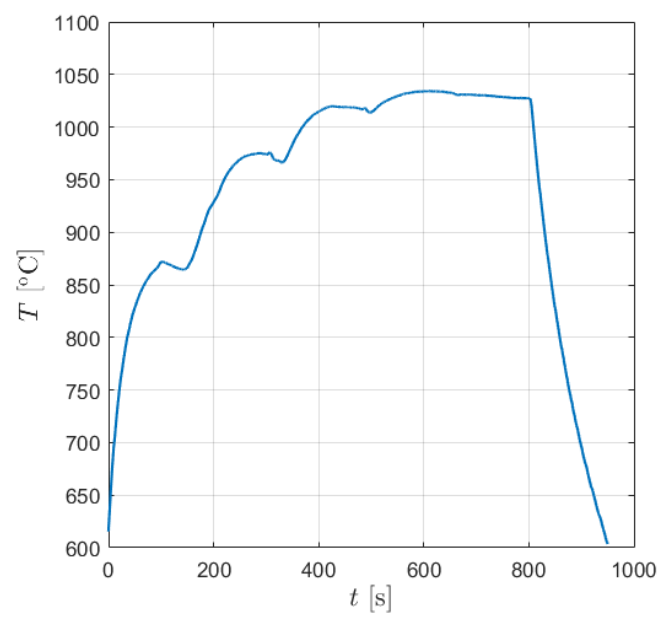

Figure 5.17: Transient heating curve during active stub tuning. The optimal stub depths through experiment that were found were; 20, 0 and 11 turns for stubs 1,2 and 3 respectively.

The first stub protrusion depth nearly matched model prediction. The opti- 
mised second and third stub protrusions were however not predicted correctly by the model. This is likely due to the 'isolated' nature of the computational model where no circulator, magnetron or terminator are present. This would affect in which manner microwaves resonate throughout the entire transmission line, and could be the main reason for the discrepancy between model and experiment. However, the maximum spot temperatures $T_{\text {steady }}$ that was reached by the optimised stub position process of Figure 5.17 was in excellent agreement with the modelled steady state temperature in Figure 5.16.

The diffusion of heat energy is also observed in Figure 5.17 where the temperature slightly relaxes over time (after $t=600 \mathrm{~s}$ ); this is in agreement with model behaviour on diffusing heat energy after rapid heating, observed in Figure 5.7. At $t=800 \mathrm{~s}$ in Figure 5.17, the magnetron is deactivated and radiative cooling rapidly cools the ironsand and $\mathrm{BN}$ tube. The ironsand was removed from the BN tube once it had cooled to room temperature, and no darkening of the part of the BN tube what was in contact with the ironsand was observed, further exemplifying the repeatability of these types of experiments.

\subsubsection{Transient models due to stub optimisation}

The optimised stub positions for low temperature heating, and maximising the steady-state temperatures were established in Figures 5.11 and 5.15, and the high temperature optimised combination of stub depths was tested against experiment in Figure 5.17. The transient heating curves were then simulated with the optimised stub positions for low and high temperature heating, and compared to the best-fitted informed model (yellow curve in Figure 5.7).

In Figure 5.18, those various optimised stub arrangements were tested against one another. A key note from this plot was the very early occurrence of $t_{r e s}$ when the system is optimised for low temperature heating (orange line, Figure 5.18). This indicated that $t_{r e s}$ was not determined only by the transition of the bulk ironsand through its Curie temperature, but also heavily influenced by a significant onset of microwave absorption at specific locations within the column of ironsand in the cavity (as the heat energy had not yet had time to diffuse throughout the ironsand). This transition through $t_{r e s}$ is also sharper in this model (orange line, Figure 5.18) where the system is optimised for low temperature heating. The stub arrangement for a maximised steadystate temperature (yellow line, Figure 5.18) showed a highly delayed $t_{\text {res }}$ when 

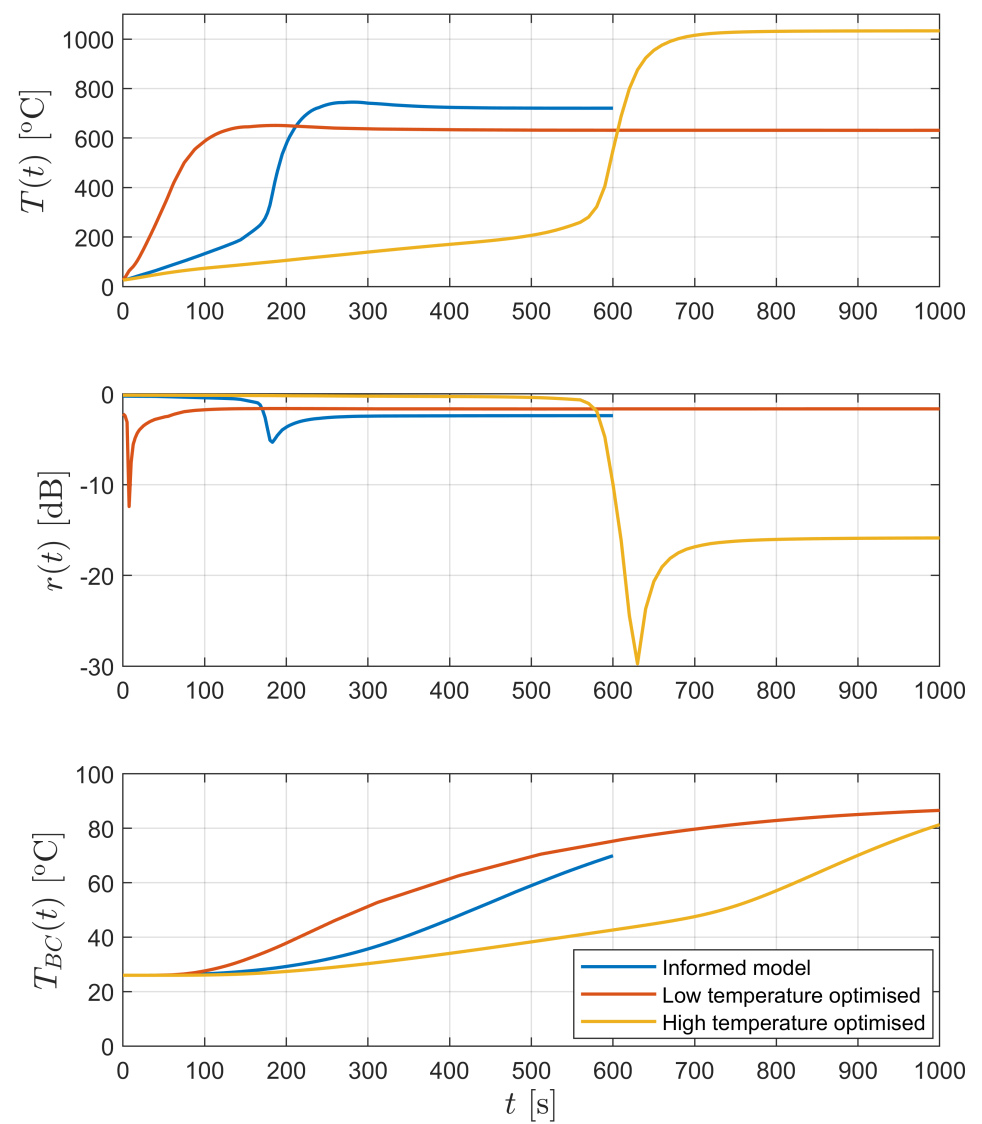

Figure 5.18: Transient heating curves of spot temperature $T(t)$, reflection coefficient $r(t)$ and temperature at end of $\mathrm{BN} \operatorname{rod} T_{B C}(t)$ upon various stub tuner configurations. The tuner depth configurations were prepared as in Figure 5.11 and Figure 5.15. Note that the time axis is here extended to $t=1000 \mathrm{~s}$.

compared to the refined model without stub depth adjustment (blue line). This is due to higher reflection of microwave energy due to the poor arrangement of stub positions for low temperature heating. This shows that the ideal stub positions at low temperatures, and high temperatures are independent. This is likely due to the difference of nature of ironsand microwave absorption at low and at high temperatures (magnetic losses at low $T$ and electrical losses at high $T$ ). A table summarising the key features of Figure 5.18 is shown.

Table 5.6 indicates the high impact that tuning the stubs correctly can have on the performance of the microwave furnace. The results of this model are qualitatively representative of experimental observation, where the microwave heating can be choked at low temperatures by poor stub arrangements, resulting in low microwave power transfer rates. The stub positions can be 


\begin{tabular}{||c|c|c|c||}
\hline Parameter & Informed model & Low temperature & High temperature \\
\hline \hline Stub turns & $0,0,0$ & $1,9,21$ & $21,9,0$ \\
\hline \hline$r\left(T_{\text {room }}\right)$ & $-0.25 \mathrm{~dB}$ & $-2.20 \mathrm{~dB}$ & $-0.14 \mathrm{~dB}$ \\
\hline$T_{\text {steady }}$ & $721^{\circ} \mathrm{C}$ & $631^{\circ} \mathrm{C}$ & $1033^{\circ} \mathrm{C}$ \\
\hline$r\left(T_{\text {steady }}\right)$ & $-2.40 \mathrm{~dB}$ & $-1.65 \mathrm{~dB}$ & $-15.87 \mathrm{~dB}$ \\
\hline$t_{\text {res }}$ & $183 \mathrm{~s}$ & $7.5 \mathrm{~s}$ & $630 \mathrm{~s}$ \\
\hline$r\left(t_{\text {res }}\right)$ & $-12.4 \mathrm{~dB}$ & $-5.3 \mathrm{~dB}$ & $-29.7 \mathrm{~dB}$ \\
\hline
\end{tabular}

Table 5.6: Table of model results of Figure 5.18 upon changing stub depths (noted in order of first, second and third stub turns), optimised to low temperature heating, and a maximised steady-state temperature, compared to the informed model from Figure 5.7.

highly optimised to maximise heat transfer to the ironsand, or for temperature control within the cavity. Automatic impedance matching algorithms have been proposed [100], demonstrating the ability to automatically tune the three-stub tuner stubs to maximise power transfer to a load. This works regardless of the load's electronic properties and could find an implementation in further work.

\subsection{Ironsand temperature profile}

It must be noted, however, that the temperatures as drawn in the figures throughout the refinement and procedure section were the temperatures at the surface of the BN tube containing the ironsand itself. Since the surface of the $\mathrm{BN}$ tube was modelled by the computational model, approximate temperatures within the ironsand itself could be 'inferred'. The axial temperature profile over time is the first property of interest.

At lower temperatures, bi-modal (two locations of greatest heating) heating is clearly visible in Figure 5.19. The transition of this bi-modal temperature profile to a central maximum occurs around $t_{\text {res }}$, accompanied by a remarkable increase in temperature of the ironsand due to the onset of significant electrical losses. The uniformity of the temperature distribution on the central axis of the ironsand is then considered. Ideally, the temperature profile would have perfect cylindrical symmetry, meaning that the ironsand has an even temperature at a specific radius from the centre of the cavity. 


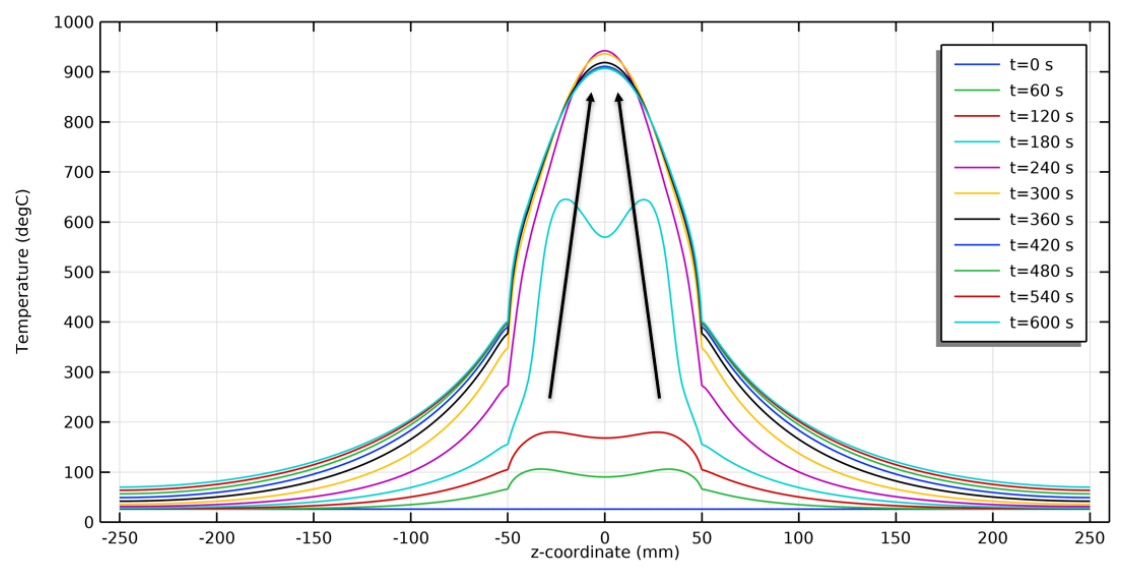

Figure 5.19: Temperature along central axis of cavity shown for 60 second intervals of the improved model with factors $\alpha=0.9, \beta=0.55, \gamma=1.3$ established and taken from the same simulation as Figure 5.7. The black arrows exemplify the displacement of the point of greatest temperature along the central axis throughout the simulation.

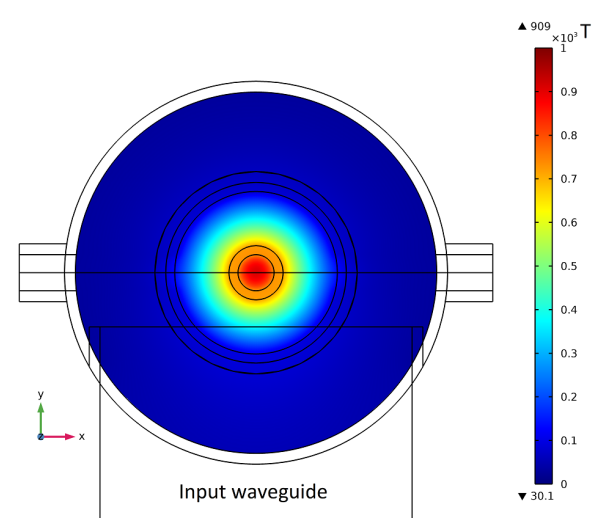

Figure 5.20: Planar cut ( $\mathrm{x}-\mathrm{y}$ plane) at middle of cavity associated temperature surface plot at $t=600$ with factors $\alpha=0.9, \beta=0.55, \gamma=1.3$ from Figure 5.7. The rectangles on the left and right are the optical access tubes, and below the cavity is the input waveguide.

The radial temperature profile of the ironsand in Figure 5.20 is almost ideally cylindrically symmetric. Figure 5.20 also demonstrates that the centre temperature of the ironsand is predicted to be $909^{\circ} \mathrm{C}$, and reduces by $170^{\circ} \mathrm{C}$ at the interface to the inner surface of the BN tube. The outer surface of the $\mathrm{BN}$ is $719^{\circ} \mathrm{C}$. Figure 5.19 shows the temperature at the centre of the ironsand column is significantly higher when compared to the ends of that column (where 
the cavity terminates). In Figure 5.21, the transient ironsand centre point temperature, surface (of BN tube) point temperature and ironsand average temperatures are compared to one another.

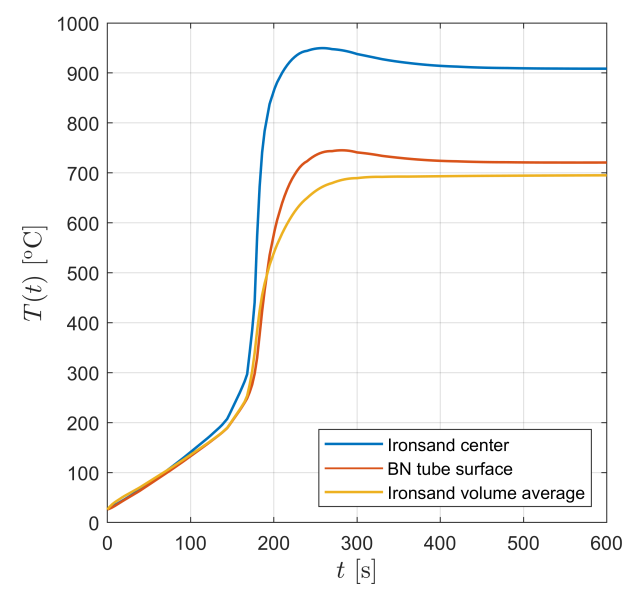

Figure 5.21: Transient temperature-time curves for temperatures at the centre of ironsand, surface of BN tube, and the volume average temperature of the ironsand, with data taken from the same dataset that produced the best-fit curve in Figure 5.7 with $\alpha=0.9, \beta=0.55$ and $\gamma=1.3$.

It must be carefully noted that the ironsand is not uniformly heated throughout the entire heating process. The ironsand volume average in Figure 5.21 initially leads the central and surface temperature measurements. This is because the system primarily heats sections of the ironsand above and below the centre of the cavity. This effect can be seen in Figure 5.19. However, the evaluated temperatures at the centre of the ironsand and the surface of the $\mathrm{BN}$ tube are very close to one another until $t_{r e s}$, after which they diverge, displayed in Figure 5.21. Near and after the transition through $t_{r e s}=184 \mathrm{~s}$, the central point in the cavity is heated the most, promoting this divergence in evaluated temperatures at the centre of the ironsand (blue), ironsand volume average (yellow) and BN tube surface temperature (orange), seen in Figure 5.21. Significantly more thermal power is lost through radiation from the surface of the bored $\mathrm{BN}$ rod in the centre of the cavity, than is conducted along the $\mathrm{BN}$ rod. The observation that the $\mathrm{BN}$ spot temperature is higher than the volume average was unexpected, but understood if Figure 5.19 is again reviewed. The temperature profile along the central axis of the cavity is highly focused to the centre of the cavity, with the temperature at the ends of the cavity barely 
exceeding $400^{\circ} \mathrm{C}$.

In summary, this chapter showed the ability to model the heating dynamics of ironsand in a custom-built microwave furnace. Some discrepancies between model and experiment are still clear, for example the disagreement in reflection coefficient $r(t)$ at low and high temperatures and the rate of change of $T_{B C}$ near $t_{r e s}$ where the model heats to the steady-state temperature much faster than experiment. However, the refinement process greatly improved agreement between model and experiment. COMSOL can be used to infer qualitative information about the temperature-profile in the cavity once steady-state temperatures are reached. This completes the tasks set out to resolve the aims of this thesis. 


\section{Chapter 6}

\section{Conclusions and recommendations}

This chapter concludes and summarises the work done throughout this thesis before outlining potential avenues for future work. The implications of the experimental and computational results of this thesis are then stated.

\subsection{Conclusions}

The core aim of this thesis was to design and build a prototype microwave furnace, and to develop a computational model which simulates the heating of TTM ironsand. The transient heating of the ironsand was simulated for a small BN tube, and the COMSOL model was refined to improve agreement with experimental results.

From the initial model development in Chapter 3, a safe-to-operate prototype microwave cavity was modelled. From the prototype design, the cavity was built in house by John Futter and tested with various ironsand microwave load geometries such as filled Alumina crucibles, a filled bored rod of BN, and a mostly-filled large BN tube. Experimental results included the production of Lichtenberg figures, partial microwave sintering and plasma production as consequences of the microwave heating. Following the experimental procedure developed specifically for this research, the ironsand was heated for 600 seconds and the surface of the $\mathrm{BN}$ tube holding the ironsand reached $716^{\circ} \mathrm{C}$, as shown in Figure 5.1).

The heating procedure was then simulated in COMSOL, producing the results shown in Figure 5.4. Refinements to the model parameters resulted in 
acceptable agreement with experimental results, as shown in Figure 5.7. The experimental reflection coefficient measurements agreed qualitatively with modelling software. Quantitatively, the magnitude of the RF power measurement results did not agree with experimental results, as seen clearly in Figure 5.7. The experimental measurement of the amplitudes of the propagating RF power through the transmission line was difficult. The microwave safe operation of the furnace designed in this work through use of BC waveguides was shown. The most likely source of disagreement between model and experiment was the nature of the ironsand. In the computational model, the ironsand was modelled as a bulk material with relative density scaled ironsand thermal and electrical properties. In reality, the ironsand is a powdered material, and therefore, exhibits significantly different thermal properties when compared to bulk TTM.

The computational model was then used to predict the optimised positions for the stubs of the three-stub tuner for both low and steady-state temperatures. It was found that protruding or retracting the stubs of the three-stub tuner significantly affected the performance of the microwave furnace, from'choking the input microwave power, to improving microwave power transfer dynamics to allow the surface of the $\mathrm{BN}$ tube in the cavity to reach over $1000^{\circ} \mathrm{C}$, as demonstrated in Figure 5.17. At high temperatures, the first stub's protrusion depth was in agreement with experiment; however, the other two stub depth predictions were not in agreement with experimental results.

\subsection{Recommendations for future work}

This research indicates the ability for microwave power to be used to heat TTM ironsand to high temperatures. This thesis also demonstrates that a computational model is a viable method to model the heating characteristics of the system, but will need further requirement. There are a range of questions which may stimulate further research for the furnace beyond this project.

1. How would the microwave furnace perform if more microwave power at $2.45 \mathrm{GHz}$ is employed, and the tuning of the stubs of the three-stub tuner is automated to optimise power transfer?

2. What would the dynamics of the system be if ironsand pellets are used instead of the powdered ironsand, as in this work? Then, how would this change upon an inclusion of hydrogen gas? 
3. Could the microwave furnace be used as a sintering device for producing 'rods' of sintered ironsand from the raw material itself? Or, would it be useful as a sintering device for the pellets?

Thus, from these questions some suggestions for future research using microwave energy to heat ironsand are made:

1. Develop and implement methods of automatically tuning the three-stub tuner as a response to the heating dynamics in the furnace.

2. Measure the intrinsic material properties of NZ ironsand over the relevant temperature ranges. Model the heating dynamics of pellets in a larger $\mathrm{BN}$ tube. Investigate how adding Hydrogen gas to the system will change heating performance throughout the reduction process.

3. Research the sintering performance of ironsand powder and/or pellets in the microwave furnace under a $\mathrm{N}_{2}$ gas sheath.

\subsection{Implications of this thesis}

It has been shown that a $2.45 \mathrm{GHz}$ microwave cavity can be used as a furnace to heat ironsand to over $1000^{\circ} \mathrm{C}$, within a few minutes, with a $1 \mathrm{~kW}$ magnetron. The three-stub tuner can be used for fine temperature control by greatly increasing or reducing the power transfer to the ironsand.

A limitation of the developed microwave furnace is its reliance on the high absorption performance of the ironsand. As the ironsand is reduced, its microwave absorption characteristics at high temperatures change, potentially worsening microwave absorption. The reduction reactions from the ironsand towards purified iron are also endothermic.

Based on the work done in this thesis, the next stages will be to investigate the continuous feeding of ironsand through the furnace. Future work should also improve understanding on the hydrogen reduction of ironsand in a system with continuous throughput, with a target processing rate aim of $1 \mathrm{~kg} / \mathrm{hr}$. 


\section{Bibliography}

[1] Ronald Frank Tylecote. A history of metallurgy. 1992.

[2] World Steel Association. World steel in figures. World Steel Association: Brussels, Belgium, 2019.

[3] World Steel Association. Fact sheet: energy use in the steel industry. Worldsteel Committee on Economic Studies Brussels, Brussels, 2016.

[4] Ottmar Edenhofer, Ramón Pichs-Madruga, Youba Sokona, Ellie Farahani, Susanne Kadner, Kristin Seyboth, A. Adler, I. Baum, S. Brunner, and P. Eickemeier. Contribution of Working Group III to the Fifth Assessment Report of the Intergovernmental Panel on Climate Change. Climate Change, pages 1-11, 2014.

[5] M. Abdul Quader, Shamsuddin Ahmed, S. Z. Dawal, and Y. Nukman. Present needs, recent progress and future trends of energy-efficient UltraLow Carbon Dioxide (CO2) Steelmaking (ULCOS) program. Renewable and Sustainable Energy Reviews, 55:537-549, March 2016.

[6] Chunbao (Charles) Xu and Da-qiang Cang. A Brief Overview of Low CO2 Emission Technologies for Iron and Steel Making. Journal of Iron and Steel Research, International, 17(3):1-7, March 2010.

[7] Pasquale Cavaliere. Clean Ironmaking and Steelmaking Processes: Efficient Technologies for Greenhouse Emissions Abatement. In Clean Ironmaking and Steelmaking Processes, pages 1-37. Springer, 2019.

[8] Chuan Wang, Christer Ryman, and Jan Dahl. Potential CO2 emission reduction for $\mathrm{BF}-\mathrm{BOF}$ steelmaking based on optimised use of ferrous burden materials. International Journal of Greenhouse Gas Control, 3(1):29-38, 2009. Publisher: Elsevier. 
[9] Terry Norgate and David Langberg. Environmental and economic aspects of charcoal use in steelmaking. ISIJ international, 49(4):587-595, 2009. Publisher: The Iron and Steel Institute of Japan.

[10] Valentin Vogl, Max Ahman, and Lars J. Nilsson. Assessment of hydrogen direct reduction for fossil-free steelmaking. Journal of Cleaner Production, 203:736-745, 2018. Publisher: Elsevier.

[11] Zhe Wang, David Pinson, Sheng Chew, Harold Rogers, Brian Joseph Monaghan, and Guangqing Zhang. Interaction of New Zealand ironsand and flux materials. ISIJ International, pages ISIJINT-2015, 2016. Publisher: The Iron and Steel Institute of Japan.

[12] Eungyeul Park, Sang-Beom Lee, Oleg Ostrovski, Dong-Jun Min, and Chang-Hee Rhee. Reduction of the mixture of titanomagnetite ironsand and hematite iron ore fines by carbon monoxide. ISIJ international, 44(1):214-216, 2004. Publisher: The Iron and Steel Institute of Japan.

[13] Yi-ran Liu, Jian-liang Zhang, Zheng-jian Liu, and Xiang-dong Xing. Phase transformation behavior of titanium during carbothermic reduction of titanomagnetite ironsand. International Journal of Minerals, Metallurgy, and Materials, 23(7):760-768, 2016. Publisher: Springer.

[14] Eungyeul Park. Gaseous Reduction of Titanomagnetite Ironsand. page 186, 2002.

[15] M. E. Fleet. The structure of magnetite. Acta Crystallographica Section B: Structural Crystallography and Crystal Chemistry, 37(4):917-920, 1981. Publisher: International Union of Crystallography.

[16] Michael E. Fleet. The structure of magnetite: Symmetry of cubic spinels. Journal of Solid State Chemistry, 62(1):75-82, 1986. Publisher: Elsevier.

[17] W. H. Bragg. The structure of magnetite and the spinels. Nature, 95(2386):561-561, 1915. Publisher: Nature Publishing Group.

[18] Barry A. Wechsler, Donald H. Lindsley, and Charles T. Prewitt. Crystal structure and cation distribution in titanomagnetites (Fe3-xTixO4). American Mineralogist, 69(7-8):754-770, 1984. Publisher: Mineralogical Society of America. 
[19] Zhengde Pang, Xuewei Lv, Yuyang Jiang, Jiawei Ling, and Zhiming Yan. Blast furnace ironmaking process with super-high TiO 2 in the slag: viscosity and melting properties of the slag. Metallurgical and Materials Transactions B, 51(2):722-731, 2020. Publisher: Springer.

[20] Haoyan Sun, Ajala Adewole Adetoro, Zhen Wang, Feng Pan, and Li Li. Direct reduction behaviors of titanomagnetite ore by carbon monoxide in fluidized bed. ISIJ International, 56(6):936-943, 2016. Publisher: The Iron and Steel Institute of Japan.

[21] Ao Zhang, Brian J. Monaghan, Raymond J. Longbottom, Mohammad Nusheh, and Chris W. Bumby. Reduction Kinetics of Oxidized New Zealand Ironsand Pellets in H 2 at Temperatures up to 1443 K. Metallurgical and Materials Transactions B, 51(2):492-504, 2020. Publisher: Springer.

[22] Sigit W. Prabowo, Raymond J. Longbottom, Brian J. Monaghan, Diego Del Puerto, Martin J. Ryan, and Chris W. Bumby. Sticking-Free Reduction of Titanomagnetite Ironsand in a Fluidized Bed Reactor. Metallurgical and Materials Transactions B, 50(4):1729-1744, 2019. Publisher: Springer.

[23] Oscar Bjareborn, Tanzeel Arif, Brian Monaghan, and Chris W Bumby. Fate of titanium in alkaline electro-reduction of sintered titanomagnetite. Materials Research Express, 7(10):106508, October 2020.

[24] Masahiro Hotta, Miyuki Hayashi, and Kazuhiro Nagata. High temperature measurement of complex permittivity and permeability of $\mathrm{Fe} 3 \mathrm{O} 4$ powders in the frequency range of 0.2 to $13.5 \mathrm{GHz}$. ISIJ international, 51(3):491-497, 2011. Publisher: The Iron and Steel Institute of Japan.

[25] Sanjay Kumar and Saurabh Shukla. Concepts and Applications of MICROWAVE ENGINEERING. PHI Learning, April 2014.

[26] Michael Vollmer. Physics of the microwave oven. Physics Education, 39(1):74, 2004. Publisher: IOP Publishing.

[27] A. C. Metaxas. Microwave heating. Power Engineering Journal, 5(5):237247, 1991. Publisher: IET. 
[28] Lixin Tang, Yue Zhao, and Jiyin Liu. An improved differential evolution algorithm for practical dynamic scheduling in steelmaking-continuous casting production. IEEE Transactions on Evolutionary Computation, 18(2):209-225, 2013. Publisher: IEEE.

[29] Lixin Tang, Jiyin Liu, Aiying Rong, and Zihou Yang. A mathematical programming model for scheduling steelmaking-continuous casting production. European Journal of Operational Research, 120(2):423-435, 2000. Publisher: Elsevier.

[30] Hubert Missbauer, Wolfgang Hauber, and Werner Stadler. A scheduling system for the steelmaking-continuous casting process. A case study from the steel-making industry. International Journal of Production Research, 47(15):4147-4172, 2009. Publisher: Taylor \& Francis.

[31] Dario Pacciarelli and Marco Pranzo. Production scheduling in a steelmaking-continuous casting plant. Computers 8 chemical engineering, 28(12):2823-2835, 2004. Publisher: Elsevier.

[32] James Clerk Maxwell. VIII. A dynamical theory of the electromagnetic field. Philosophical transactions of the Royal Society of London, (155):459512, 1865. Publisher: The Royal Society London.

[33] Oliver Heaviside. Electromagnetic Theory. "The Electrician" printing and publishing Company, limited, 1893.

[34] Joseph John Thomson. Notes on recent researches in electricity and magnetism: intended as a sequel to Professor Clerk-Maxwell's Treatise on electricity and magnetism. Clarendon Press, 1893.

[35] Lord Rayleigh. XVIII. On the passage of electric waves through tubes, or the vibrations of dielectric cylinders. The London, Edinburgh, and Dublin Philosophical Magazine and Journal of Science, 43(261):125-132, 1897. Publisher: Taylor \& Francis.

[36] David J. Griffiths. Introduction to Electrodynamics. Prentice Hall New Jersey, 1962.

[37] Harald Ibach and Hans Lüth. Solid-state physics: an introduction to theory and experiment. Springer Science \& Business Media, 2012. 
[38] George Lawrence Matthaei, Leo Young, and Edward McClung Thompson Jones. Microwave filters, impedance-matching networks, and coupling structures. Artech house, 1980.

[39] Allan W. Scott. Understanding Microwaves. Springer Science \& Business Media, 1993.

[40] David R. Lide. CRC handbook of chemistry and physics, volume 85. CRC press, 2004.

[41] John Henry Poynting. XV. On the transfer of energy in the electromagnetic field. Philosophical Transactions of the Royal Society of London, (175):343-361, 1884. Publisher: The Royal Society London.

[42] Friedrich Wilhelm Bessel. Untersuchung des Theils der planetarischen Störungen, welcher aus der Bewegung der Sonne entsteht. 1824.

[43] Max Planck. The Theory of Thermal Radiation. Ann. Phys, 4, 1914.

[44] Jean Baptiste Joseph baron Fourier. Théorie analytique de la chaleur. Chez Firmin Didot, père et fils, 1822.

[45] Isaac Newton. VII. Scala Graduum Caloris. Philosophical Transactions of the Royal Society of London, 22(270):824-829, 1701. Publisher: Royal Society.

[46] COMSOL Multiphysics. RF Module User's Guide. COMSOL Multiphysics, 2020.

[47] H P R Frederickse. Characterization of microwave window materials. page 30, 1982.

[48] Susumu Takahashi, Yusuke Imai, Akinori Kan, Yuji Hotta, and Hirotaka Ogawa. Dielectric and thermal properties of isotactic polypropylene/hexagonal boron nitride composites for high-frequency applications. Journal of Alloys and Compounds, 615:141-145, December 2014.

[49] P. Beiss, R. Ruthardt, and H. Warlimont, editors. Powder Metallurgy Data. Refractory, Hard and Intermetallic Materials, volume 2A2 of Landolt-Börnstein - Group VIII Advanced Materials and Technologies. Springer-Verlag, Berlin/Heidelberg, 2002. 
[50] Pawel Rutkowski, Karol Gala, Kamila Misiura, and Jan Huebner. The Influence of Thermal Properties Anisotropy on Subtractive Laser Processing of B4C/h-BN Composites. Materials, 13(22):5191, 2020. Publisher: Multidisciplinary Digital Publishing Institute.

[51] D.P. DeWitt and Y.S. Touloukian. Thermal Radiative Properties of Nonmetallic Solids, volume 8. Plenum publishing, 1972.

[52] Zircar Ceramics. AL-25-1700.pdf, 2016.

[53] Zircar Ceramics. Permittivity on a range of ZIRCAR Ceramics Alumina Products, 2019.

[54] Richard J. Harrison and Andrew Putnis. The magnetic properties and crystal chemistry of oxide spinel solid solutions. Surveys in Geophysics, 19(6):461-520, 1998. Publisher: Springer.

[55] Juris Meija, Tyler B Coplen, Michael Berglund, Willi A Brand, Paul De Bièvre, Manfred Gröning, Norman E Holden, Johanna Irrgeher, Robert D Loss, Thomas Walczyk, et al. Atomic weights of the elements 2013 (iupac technical report). Pure and Applied Chemistry, 88(3):265-291, 2016.

[56] Richard F Mould. Pierre curie, 1859-1906. Current oncology, 14(2):74-82, 2007.

[57] Gerald Schubert. Treatise on geophysics. Elsevier, 2015.

[58] Soshin Chikazumi and Chad D. Graham. Physics of Ferromagnetism 2e. Number 94. Oxford University Press on Demand, 2009.

[59] Davide Levy, Roberto Giustetto, and Andreas Hoser. Structure of magnetite (fe 3 o 4 ) above the Curie temperature: a cation ordering study. Physics and Chemistry of Minerals, 39(2):169-176, 2012.

[60] Yaroslav Grosu, Abdessamad Faik, Iñigo Ortega-Fernández, and Bruno D'Aguanno. Natural Magnetite for thermal energy storage: Excellent thermophysical properties, reversible latent heat transition and controlled thermal conductivity. Solar Energy Materials and Solar Cells, 161:170176, 2017. Publisher: Elsevier. 
[61] Charles Kittel. Introduction to solid state physics. 1976.

[62] Rochelle M. Cornell and Udo Schwertmann. The iron oxides: structure, properties, reactions, occurrences and uses. John Wiley \& Sons, 2003.

[63] Gerald Burns. Solid State Physics. Academic Press: Orlando. ISBN-13, 1985.

[64] Neil W Ashcroft and N David Mermin. Solid State Physics, 1976.

[65] Evan Pert, Yuval Carmel, Amikam Birnboim, Tayo Olorunyolemi, David Gershon, Jeff Calame, Isabel K. Lloyd, and Otto C. Wilson. Temperature measurements during microwave processing: the significance of thermocouple effects. Journal of the American Ceramic Society, 84(9):1981-1986, 2001. Publisher: Wiley Online Library.

[66] Frank Gerigk. Cavity types. arXiv:1111.4897 [physics], November 2011. arXiv: 1111.4897.

[67] Alfred W. Cook. Coaxial magnetron using the TE111 mode, 1986.

[68] Alex Gurevich. Superconducting radio-frequency fundamentals for particle accelerators. Reviews of Accelerator Science and Technology, 5:119-146, 2012 .

[69] A Nassiri, B Chase, P Craievich, A Fabris, H Frischholz, J Jacob, E Jensen, M Jensen, R Kustom, and R Pasquinelli. History and technology developments of radio frequency $(\mathrm{RF})$ systems for particle accelerators. IEEE Transactions on Nuclear Science, 63(2):707-750, 2015.

[70] Waldemar Scharf. Particle accelerators and their uses. CRC Press, 1986.

[71] JL Vossen and JJ O'Neill Jr. Rf sputtering processes. Technical report, RCA Labs., Princeton, NJ, 1968.

[72] PD Davidse and LI Maissel. Dielectric thin films through RF sputtering. Journal of Applied Physics, 37(2):574-579, 1966.

[73] John H Keller and WB Pennebaker. Electrical properties of RF sputtering systems. IBM Journal of Research and Development, 23(1):3-15, 1979. 
[74] PJM Carrott, JMV Nabais, MML Ribeiro Carrott, and JA Menéndez. Thermal treatments of activated carbon fibres using a microwave furnace. Microporous and Mesoporous Materials, 47(2-3):243-252, 2001.

[75] Chao Zhao, Jef Vleugels, C Groffils, PJ Luypaert, and Omer Van der Biest. Hybrid sintering with a tubular susceptor in a cylindrical single-mode microwave furnace. Acta materialia, 48(14):3795-3801, 2000.

[76] S. F. Cleary, B. S. Pasternack, and G. W. Beebe. Cataract incidence in radar workers. Archives of Environmental Health: An International Journal, 11(2):179-182, 1965. Publisher: Taylor \& Francis.

[77] S. F. Cleary. Microwave cataractogenesis. Proceedings of the IEEE, 68(1):49-55, 1980. Publisher: IEEE.

[78] James C. Lin. Human exposure to RF, microwave, and millimeter-wave electromagnetic radiation [Health Effects]. IEEE Microwave Magazine, 17(6):32-36, 2016. Publisher: IEEE.

[79] M. Röösli, R. Rapp, and C. Braun-Fahrländer. Radio and microwave frequency radiation and health-an analysis of the literature. Gesundheitswesen (Bundesverband der Arzte des Offentlichen Gesundheitsdienstes (Germany)), 65(6):378-392, 2003.

[80] International Commission on Non-Ionizing Radiation Protection. Guidelines for limiting exposure to Electromagnetic Fields $(100 \mathrm{kHz}$ to 300 GHz). Health Physics, 118(5):483-524, 2020. Publisher: LWW.

[81] John F Gerling and P O Box. Waveguide components for configurations for Optimal Performance in Microwave Heating Systems. page 8, 2000.

[82] A. S. Gilmour. Klystrons, traveling wave tubes, magnetrons, crossed-field amplifiers, and gyrotrons. Artech House, 2011.

[83] Resonant_Cavity_Magnetron_Diagram png: Ian Dunsterderivative work: Vanessa Ezekowitz. Diagram of a resonant cavity magnetron (Accessed 3/3/2021), August 2008.

[84] C. Lester Hogan. The ferromagnetic Faraday effect at microwave frequencies and its applications. Reviews of Modern Physics, 25(1):253, 1953. Publisher: APS. 
[85] C. Lester Hogan. The ferromagnetic Faraday effect at microwave frequencies and its applications: the microwave gyrator. The Bell System Technical Journal, 31(1):1-31, 1952. Publisher: Nokia Bell Labs.

[86] H.A. Bethe. Theory of Diffaction by Small Holes. The Physical Review, 66(7-8):163-182, October 1944.

[87] V. A. Lavrenko and A. F. Alexeev. High-temperature oxidation of boron nitride. Ceramics international, 12(1):25-31, 1986. Publisher: Elsevier.

[88] Steve Winder and Joseph Carr. Newnes radio and RF engineering pocket book. Newnes, 2002.

[89] E. Brodu and M. Balat-Pichelin. Emissivity of boron nitride and metals for the solar probe plus mission. Journal of Spacecraft and Rockets, 53(6):1119-1127, 2016. Publisher: American Institute of Aeronautics and Astronautics.

[90] José A. Bittencourt. Fundamentals of plasma physics. Springer Science \& Business Media, 2013.

[91] Francis F. Chen. Introduction to plasma physics. Springer Science \& Business Media, 2012.

[92] John M. Meek and John Drummond Craggs. Electrical breakdown of gases. 1978 .

[93] Alice Hong. Dielectric strength of air. The Physics Factbook, 2000.

[94] Pradyot Patnaik. Handbook of inorganic chemicals, volume 529. McGrawHill New York, 2003.

[95] E. J. Essene and D. C. Fisher. Lightning strike fusion: extreme reduction and metal-silicate liquid immiscibility. Science, 234(4773):189-193, 1986. Publisher: American Association for the Advancement of Science.

[96] Akihiro Kawashima and Seisaburo Hoh. Lichtenberg figures on various electrical insulating materials. IEEE Transactions on Electrical Insulation, (1):51-56, 1978. Publisher: IEEE. 
[97] Michael Cherington, Gilbert McDonough, Sheryl Olson, Rick Russon, and Philip R. Yarnell. Lichtenberg figures and lightning: case reports and review of the literature. CUTIS-NEW YORK-, 80(2):141, 2007. Publisher: QUADRANT HEALTH COM INC.

[98] Randall M. German. Sintering theory and practice. 1996.

[99] Charles Francis Curtiss and Joseph O. Hirschfelder. Integration of stiff equations. Proceedings of the National Academy of Sciences of the United States of America, 38(3):235, 1952. Publisher: National Academy of Sciences.

[100] Shimiao Lai, Jingxin Qiao, Nouman Rasool, Kang Li, Huacheng Zhu, and Yang Yang. A dynamic impedance matching algorithm of three-stub tuners based on equivalent circuit analysis. Journal of Microwave Power and Electromagnetic Energy, 54(4):330-347, 2020.

[101] Jean Le Rond d'Alembert. Sur la Courbe Que forme une Corde. Ambroise Haude, 1749. 


\section{List of Figures}

2.1 A model of a rectangular WR340 waveguide with width $86.36 \mathrm{~mm}$ and height $43.18 \mathrm{~mm}$ built in COMSOL Multiphysics. In this work, $2.45 \mathrm{GHz}$ radiation is launched into the bottom-right of the model. This microwave power is propagated through the waveguide in the characteristic $\mathrm{TE}_{10}$ mode profile, and out the other side of the waveguide (which cannot be seen in this image). 11

2.2 A cut-open view of a closed 3D resonant cavity model with radius $R$ and length $L$ built in COMSOL Multiphysics. For any specific frequency, various combinations of radii and length result in resonant modes within the cavity. . . . . . . . . . . . 14

2.3 Plot showing combinations of cylindrical cavity radii and lengths which produce electromagnetic resonant modes at fixed frequency of $2.45 \mathrm{GHz}$. The lines (combinations of resonant cavity dimensions) are calculated directly from (Eqs. 2.2.6, 2.2.7a, 2.2.7b). The curves in this figure are exactly analogous for the righthanded resonance cylinder found in [38]. . . . . . . . . . . . . 17

2.4 Example of tetrahedral meshing used in COMSOL Multiphysics for Figure 2.2. In this example, the dimensions of a cylindrical resonant cavity are $R=68 \mathrm{~mm}$ and $L=72 \mathrm{~mm}$. . . . . . . . . 21

2.5 The simulated resonance curves as evaluated via (Eq. 2.4.8) for each combination of $R \in(45,120) \mathrm{mm}$ and $L \in(50,150) \mathrm{mm}$. . 26

2.6 Picture of BN rod-turned-crucible used in this research. The drilled hole is $100 \mathrm{~mm}$ long and has a diameter of $10 \mathrm{~mm}$. The tube diameter is $15 \mathrm{~mm}$ and its length is $300 \mathrm{~mm} \ldots \ldots$. . . . 27 
2.7 Relevant temperature dependent material properties of BN used in modelling calculations (Top left; complex permittivity $\varepsilon_{r}^{\prime}(T)$, $\varepsilon_{r}^{\prime \prime}(T)$, top right; emittance $\epsilon(T)$, bottom left; heat capacity $c_{p}(T)$, bottom right; thermal conductivity $\left.k_{t}(T)\right) . \ldots . . .28$

2.8 Half-inch thick AL-25/1700 alumina fibreboard annuli cut-outs stacked on paper towel before placement into microwave cavity.

2.9 Cup of TTM ironsand, exemplifying the black colour of the ironsand. The lighter specks in this figure are impurities in the ironsand. This powder was filled into the BN tube from Figure 2.6 for experiments. . . . . . . . . . . . . . . . . 30

2.10 Temperature dependent values used in the initial model for TTM ironsand adapted from literature $[24,60]$ values for magnetite (Top left; complex permittivity $\varepsilon_{r}^{\prime}(T), \varepsilon_{r}^{\prime \prime}(T)$, top right; complex permeability $\mu_{r}^{\prime}(T), \mu_{r}^{\prime \prime}(T)$, bottom left; heat capacity $c_{p}(T)$, bottom right; thermal conductivity $\left.k_{t}(T)\right) \ldots \ldots$. . . . . 31

3.1 Models of empty resonant cavities with input WR340 waveguides in (a) horizontal; and (b) vertical input orientations. The orange sections are the copper parts of the cavity, and grey corresponds to air. . . . . . . . . . . . . . . . . 35

3.2 A cut-open view of an empty 3D resonant cavity model with radius $R$ and length $L$ built in COMSOL Multiphysics with two $50 \mathrm{~mm}$ (diameter) holes cut from the ends of the cavity (the top hole was hidden for the purposes of this picture). . . . . . . . 36

3.3 The closed initial design of the microwave furnace. A horizontal input WR340 waveguide is included (width $86.36 \mathrm{~mm}$ ) in addition to two $\mathrm{BC}$ waveguide tubes with radius $25 \mathrm{~mm}$ and length $200 \mathrm{~mm}$. The cavity dimensions for this figure are $R=68 \mathrm{~mm}$ and $L=72 \mathrm{~mm}$, but were not the final combination of dimensions chosen. . . . . . . . . . . . . . . . . . . 38 
3.4 Average EM energy density integrals (Eq. 2.4.8) for a variety of cavity radii and lengths with horizontal (left) and vertical (right) input waveguide. The model in Figure 3.3 was used for this simulation where the cavity length and radius are varied. Neither insulation or BN tubes were included in this parameter sweep, however the $50 \mathrm{~mm}$ diameter $\mathrm{BC}$ waveguide tubes were included. . . . . . . . . . . . . . . . . . . 39

3.5 Same parameter sweep as Figure 3.4, however of the average resonance quality, the centralised resonance quality factor $\zeta(R, L)$ from (Eq. 3.4.1) is visualised here. . . . . . . . . . . . . . . . 40

3.6 Arrow volume plot of the resulting resonance in the cavity with $R=50 \mathrm{~mm}$ and $L=100 \mathrm{~mm}$. The WR340 input waveguide has characteristic $\mathrm{TE}_{10}$ mode propagation (i.e. the circulating magnetic field arrows). No thermal insulation was included in this model. The length and direction of the arrows represent the value of the electric and magnetic fields. . . . . . . . . . . . . 41

3.7 The inclusion of thermal insulation reduced the axial electromagnetic field energy density (blue), and appeared to produce a 'bi-modal' type of field energy density profile along the central axis of the cavity (orange) . . . . . . . . . . . . . . . . 43

3.8 Cross-section of prototype furnace simulation $(R=50 \mathrm{~mm}, L=$ $100 \mathrm{~mm}$ ) showing $E_{z}$ field intensity for cavity with $(3.8 \mathrm{~b})$ and without (3.8a) AL-25/1700 Alumina fibreboard annuli (inner radius $22.5 \mathrm{~mm}$ ). No BN tubes were included in this model. . . . 43

3.9 Exaggerated diagram of geometrical correction to wall depth. View is from above cavity. This diagram exemplifies the reduced effective depth of the wall due to the curvature of the cavity. A correction factor $\Delta=R+d-\sqrt{(R+d)^{2}-(h / 2)^{2}}$ was then calculated. . . . . . . . . . . . . . . 45

3.10 Exaggerated diagram of optical access limitation by pyrometer. View is from the side of cavity. A description of initial parameters in this plot is given in Table 3.2, and the rest given in Table 3.3. 46 
3.11 Diagram of optical access tube geometries resulting in a range of $2.45 \mathrm{GHz}$ microwave attenuation. The black line describes the minimum optical tube diameter, and the dashed coloured lines describe the amount of $2.45 \mathrm{GHz}$ microwave attenuation for each combination of tube dimensions. . . . . . . . . . . . . . . 47

4.1 Basic setup of RF transmission line. From right to left; magnetron launch, circulator, terminator (behind circulator), dualdirectional coupler, three-stub tuner and microwave furnace. All components are discussed, and assembled as in this picture of the setup. The system is raised from the bench by lab jacks. Water cooling tubing (blue tubes on the right) for the magnetron, terminator and circulator is included. Note that the optical access tubes had not yet been added to the side of the microwave cavity. 50

4.2 Cross-sectional diagram of typical magnetron structure. The magnetic field lines are parallel with the axis of the structure (perpendicular to the page) [83]. . . . . . . . . . 51

4.3 The magnetron launch has three sections. The first of which is a heating block (top). The second is cathode-anode 2M261M32 magnetron (center). The third is a water cooling heat-sink (around the magnetron block), where the microwave power is produced. The final part is ceramic probe, which protrudes into the transmission line (Figure 4.3b). . . . . . . . . . . . . 52

4.4 The two black disks are the ferrite spinel magnets, which allow for selected directional propagation of electromagnetic radiation through the component; i.e. from IN to OUT, or from OUT to LOAD . . . . . . . . . . . . . . . . . 52

4.5 The three stubs and water tubes in the terminator are designed as a load for microwave power. This component was also used for microwave power magnitude calibration. . . . . . . . . . 54

4.6 The three stubs with spherical end caps can be retracted and protruded by turning the tuners from the outside of the component. This changes the reflection and transmission properties of incident microwave power through this component, allowing for optimised microwave heating of ironsand in the cavity. . . . . . 54 
4.7 The dark knobs in Figure 4.7 a are $50 \Omega$ terminators. The other ends (pictured here with white plastic covers) are connected to AD8317 RF-DC detectors via coaxial cables for directional power analysis. Further details on the connection of these detectors is included in Section 4.3.2. . . . . . . . . . . . . . . 56

4.8 First build of the microwave cavity by John Futter. A dozen screws were screwed in for the purpose of the picture. The input WR340 waveguide is on the right, and the dimensions of the microwave cavity and $\mathrm{BC}$ waveguides were verified and were in agreement with the commissioning outline. . . . . . . . . 57

4.9 Initial assembly of the prototype microwave furnace for preliminary testing. From left to right: alumina boat filled with ironsand, microwave furnace cavity, three-stub tuner, dual-directional coupler, terminator (above), circulator (below), magnetron. Below on the right (with the blue sticker) is the power controller on the right with continuous voltage control from $0 \rightarrow 6 \mathrm{~V}$. Below on the left are two multimeters to qualitatively measure the time-averaged directional microwave power propagating through the transmission line. . . . . . . . . . . . . . . . . . . . 58

4.10 Final microwave furnace setup picture. The optical access tubing, the water cooling jacket, kaowool plugging, and BN tubes are included in this picture, in addition to being placed vertically again.

4.11 RF receiver for initial qualitative microwave leakage measurements. The needle moved when microwave radiation interacted with the antenna on the back of the RF receiver. The functionality and sensitivity of this device was tested by observation of microwave leakage from a commercial microwave oven at the on-site cafeteria. . . . . . . . . . . . . . . . . . 61

4.12 ERD detector, and previously-calibrated power measurements for quantitative microwave leakage measurements. A linear fit (in $\mathrm{dBm}$ which is a logarithmic power measurement unit, relative to $1 \mathrm{~mW}$ ) was employed to find the power measurement beyond the calibrated range. . . . . . . . . . . . . . 62 
4.13 AD8317 RF-DC detector boards attached via adapters to the coaxial connectors on the dual-directional component of the transmission line. . . . . . . . . . . . . . . . 63

4.14 Oscilloscope screen while connected to AD8317 board output lines. The $150 \mathrm{kHz}$ signal was clearly present in this view of the signal. In addition, a $50 \mathrm{~Hz}$ mains hum was noted by the oscilloscope. . . . . . . . . . . . . . . . . 64

4.15 Pulse rate to mass flow calibration. It shows an excellent linear relationship $\Delta m / \Delta t=0.66 f_{t}+0.43$ between tick rate and mass flow rate. Note that the linear relationship is extrapolated to below 20, and above $83 \mathrm{~Hz} \ldots \ldots$. . . . . . . . . . . 66

4.16 Plot of measured backward voltage against power deposited into water. . . . . . . . . . . . . . . . 67

4.17 Pictures of 4.17a two colour QKTRD-4485, and 4.17b single colour CTLaserM2 pyrometer measurement heads. . . . . . . . . 69

4.18 Comparison of spot temperature measurements made by single colour CTLaserM2 pyrometer, and two-colour QKTRD-4485 pyrometer. For this calibration, the emissivity of $\mathrm{BN}$ is assumed to be constant near the measurement wavelengths $\left(\lambda_{1}=970 \mathrm{~nm}\right.$, $\lambda_{2}=1050 \mathrm{~nm}$ ) for the QKTRD-4485 pyrometer. . . . . . . . 70

4.19 Before-and-during pictures of plasma ignition in microwave cavity with Alumina boat filled with ironsand. A loud low-pitch sound was produced while the plasma was lit. This was due to the $50 \mathrm{~Hz}$ mains 'hum', powering the magnetron, resulting in plasma pulsing. . . . . . . . . . . . . . . . . 72

4.20 Red-hot glowing TTM ironsand and melted-end of alumina crucible in closed-ended quartz tube. Some browning of the end of the quartz tube is seen and is due to plasma deposition of materials onto the tube.

4.21 Crystallised surface of melted section of Alumina-ironsand from Figure 4.20 after rapid radiative cooling, viewed under microscope. The crystal structures shown here match the macroscopic crystalline structure of pure magnetite, meaning that the ironsand was also fully melted. . . . . . . . . . . . . . 73 
4.22 Lichtenberg figure produced in body of loose ironsand in Alumina crucible after the loose ironsand was carefully tapped off. . . . . 74

4.23 Cross section of extracted rod of partially-sintered TTM ironsand. The diameter of this rod is $14 \mathrm{~mm}$. There are two phases of ironsand; the outer which is partially melted, and the interior of the tube which is effectively sintered. . . . . . . . . . . 75

4.24 End-piece section of extracted rod of partially-sintered TTM ironsand. The diameter of the rod is $14 \mathrm{~mm}$. The internal section of this appears partially sintered. A blue hue in the ironsand is observed at the end of this partially-sintered ironsand column. . 76

4.25 XRD scans of ironsand used in this work, compared with raw ironsand. Patterns indicate that the microwave-heated ironsand in the cavity had not significantly oxidised throughout heating. The dots indicate the presence of TTM, and the circles indicate presence of TTH. The x-ray source used to obtain these diffraction patterns was Co-60. . . . . . . . . . . . . 77

4.26 Diagram of experimental setup. Microwave power is contained in the yellow blocks, the red sections being WR340 waveguide connections. Green sections are connections between various electronics. The grey connection is the optical access of the pyrometer to the resonant cavity. The blue dots on the transmission line components are where water-cooling is employed. . . . . . 78

5.1 Experimental results for the time evolution of the $\mathrm{BN}$ tube temperature $T(t)$, cavity reflection coefficient $r(t)$ and $T_{B C}(t)$. A characteristic absorption peak occurs around $t \equiv t_{\text {res }}=187 \mathrm{~s}$, indicating a sharp change of electromagnetic properties of the ironsand around its Curie temperature. The grey lines represent the standard error of those respective measurements, calculated by error propagation. . . . . . . . . . . . . . . 82 
5.2 Transient evolution of transmitted and reflected power supplied to and from the cavity during an ironsand heating run. This was calculated via the power calibration done in Figure 4.3.1, in Section 4.3.3. The input power reduces from $800 \mathrm{~W}$ to $600 \mathrm{~W}$ over $600 \mathrm{~s}$. This is from the same experimental run as Figure 5.1. The yellow line is a best fit curve of the black input power curve, shown in (Eq. 5.1.1). . . . . . . . . . . . . . . . 84

5.3 An external view of the model geometry used to model heating of TTM contained within the 'thin BN tube'. The BC waveguides, white BN 'tube', optical access holes and three-stub tuner are included to best mirror the experimental setup. . . . . . . . . 87

5.4 Simulated transient plots for microwave heating of ironsand in the experimental furnace. A clear resonance occurs at $t=96 \mathrm{~s}$, which temporarily delivers a greatly increased rate of heating. . 88

5.5 Side-on view of $U_{E M}$ (Eq. 2.1.5) in cavity during model run of Figure 5.4 at various instants during transient simulation of the model. Left; $t=66 \mathrm{~s}$ : middle: $t=96 \mathrm{~s}$, right: $t=126 \mathrm{~s}$. The ironsand is located in the centre of the cavity. . . . . . . . . 89

5.6 Simulated transient plots for microwave heating of ironsand in the experimental furnace. The varied parameters $\alpha=0.9$ and $\beta=0.6$ result in improved agreement between model and experiment. The blue line is the updated model, the orange is the original model from Figure 5.4 and the yellow dashed line is experimental result. . . . . . . . . . . . . . . . . . 93

5.7 Simulated transient plots for microwave heating of ironsand in the experimental furnace. The varied parameters $\alpha, \beta$ and $\gamma$ result in a further improved agreement between model and experiment from Figure 5.6. . . . . . . . . . . . . . . 95

5.8 Various independent stub protrusions of all three stubs, resulting in a varied reflection coefficient $r\left(T_{\text {room }}\right)$. Moving stub 3 to 21 turns resulted in improved microwave absorption. . . . . . . . 97 
5.9 Various independent stub protrusions of stub 1 and 2, resulting in a further varied reflection coefficient $r\left(T_{\text {room }}\right)$ depending on stub depths. In these models, the depth of stub 3 was fixed to 21 turns. Moving stub 2 to 9 turns resulted in improved microwave absorption. . . . . . . . . . . . . . . . . . 98

5.10 Various independent stub protrusions of stub 1, resulting in a worsened reflection coefficient $r\left(T_{\text {room }}\right)$. In this model, the depth of stub 3 was fixed to 21 turns, and the depth of stub 2 was fixed to 9 turns. . . . . . . . . . . . . . . . . . 98

5.11 Cross section of WR340 waveguide section of Figure 5.3. The tuning stubs are shown in the positions optimised for low temperature heating (as predicted by the modelling in this section) with stub turns of 1, 9 and 21 respectively for stubs 1,2 and 3. . 99

5.12 Various independent stub protrusions of all three stubs, resulting in a varied reflection coefficient for steady-state models. Moving stub 1 to 21 turns resulted in improved microwave absorption. . 99

5.13 Various independent stub protrusions of stubs 2 and 3, resulting in a further varied high temperature reflection coefficient depending on stub depths. In these models, the depth of stub 1 was fixed to 21 turns. Moving stub 2 to 9 turns resulted in improved microwave absorption. . . . . . . . . . . . . 100

5.14 Various independent stub protrusions of stub 3, resulting in a further varied reflection coefficient at $r\left(T_{\text {steady }}\right)$. In this model, the depth of stub 1 was fixed to 21 turns, and the depth of stub 2 was fixed to 9 turns. . . . . . . . . . . . . . . . 101

5.15 Cross section of WR340 waveguide section of Figure 5.3. The tuning stubs are shown in the positions optimised for maximum spot temperature $T_{\text {steady }}$ (as predicted by the modelling in this section) with stub turns of 21,9 and 0 respectively for stubs 1 ,

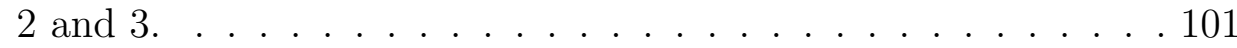

5.16 Various stub 3 depths and those associated steady-state temperatures. An increased stub depth resulted in worsened microwave absorption, and therefore a reduced $T_{\text {steady }}$. This is the same dataset from the variation of the depth of stub 3 in Figure 5.14. 102 
5.17 Transient heating curve during active stub tuning. The optimal stub depths through experiment that were found were; 20, 0 and 11 turns for stubs 1,2 and 3 respectively. . . . . . . . . . . . 102

5.18 Transient heating curves of spot temperature $T(t)$, reflection coefficient $r(t)$ and temperature at end of $\mathrm{BN} \operatorname{rod} T_{B C}(t)$ upon various stub tuner configurations. The tuner depth configurations were prepared as in Figure 5.11 and Figure 5.15. Note that the time axis is here extended to $t=1000 \mathrm{~s}$. . . . . . . . . . 104

5.19 Temperature along central axis of cavity shown for 60 second intervals of the improved model with factors $\alpha=0.9, \beta=0.55$, $\gamma=1.3$ established and taken from the same simulation as Figure 5.7. The black arrows exemplify the displacement of the point of greatest temperature along the central axis throughout the simulation. . . . . . . . . . . . . . 106

5.20 Planar cut ( $\mathrm{x}-\mathrm{y}$ plane) at middle of cavity associated temperature surface plot at $t=600$ with factors $\alpha=0.9, \beta=0.55, \gamma=1.3$ from Figure 5.7. The rectangles on the left and right are the optical access tubes, and below the cavity is the input waveguide.106

5.21 Transient temperature-time curves for temperatures at the centre of ironsand, surface of BN tube, and the volume average temperature of the ironsand, with data taken from the same dataset that produced the best-fit curve in Figure 5.7 with $\alpha=0.9$, $\beta=0.55$ and $\gamma=1.3 \ldots \ldots \ldots \ldots$. . . . . . . . . . . . . . . . .

B.1 Plot of the Bessel functions (and the derivatives thereof) of the zeroth, first and second kind. Here the location of the first few zeros as specified in Table 2.2 are apparent. . . . . . . . . . . 146

B.2 Combinations of $\mathrm{BC}$ waveguide aspect ratios, at fixed attenuation amounts (coloured curves). The maximum radius is given by the cutoff frequency equation $R\left(=c \chi_{l m}^{\prime} / 2 f_{0}\right)=35.86 \mathrm{~mm}$ from (Eq. 2.2.6). . . . . . . . . . . . . . . . . 148

D.1 Block diagram used to acquire data during research. . . . . . . 152

D.2 Standard user interface for COMSOL Multiphysics 5.6 while software is simulating a transient model. . . . . . . . . . 153

D.3 Standard user interface for MATLAB R2018a. . . . . . . . . . . 154 


\section{List of Tables}

2.1 The lowest cutoff frequencies for the $\mathrm{TE}_{\mathrm{mn}}$ modes of the rectangular waveguide. These frequencies are found by solving (Eq. 2.2.3) for $f_{m n}$ with $a=86.36 \mathrm{~mm}$ and $b=43.18 \mathrm{~mm}$. . . . . . 13

2.2 The first two zeros of $J_{l}(\Gamma r)$ and $J_{l}^{\prime}(\Gamma r)$ are tabulated with $l=0,1,2$. Note especially the low $\mathrm{TE}_{11}$ mode Bessel function solution. . . . . . . . . . . . . . . . 16

3.1 The smallest microwave cavity dimensions depending on input waveguide orientation with width $86.36 \mathrm{~mm}$ and height $43.18 \mathrm{~mm}$. These input orientations can be seen in Figures 3.1a and 3.1b. . 36

3.2 The table of relevant fixed dimensions used for the optical access hole diameter calculation. . . . . . . . . . . . . . . . . 44

3.3 The table of relevant parameters used specifically for the optical access hole calculation. . . . . . . . . . . . . . . 46

3.4 Table of commissioned dimensions to build the microwave cavity. The radii written in this table refer to the inner radius of that part. The width of the copper tubing used to build the cavity was $2 \mathrm{~mm} \ldots \ldots \ldots \ldots \ldots \ldots$. . . . . . . . . . . . . . . 48

5.1 Table of key measured data from Figure 5.1. . . . . . . . . 83

5.2 Table of key initial modelling results taken from Figure 5.4 . . . 89

5.3 Table of steady-state temperature and reflection coefficients upon variation of input power $P_{f w d}$ by factor $\alpha$. The original values for $T_{\text {steady }}$ and $r\left(T_{\text {steady }}\right)$ as from Table 5.2 are retrieved when $\alpha=1$. Note the omission of $r\left(T_{\text {room }}\right)$, this is due to the room temperature reflection not depending on the magnitude of the input power. . . . . . . . . . . . . . . . 91 
5.4 Table of steady-state temperature and reflection coefficients upon variation of ironsand packing fraction by factor $\beta$. The original values for $T_{\text {steady }}$ and $r\left(T_{\text {steady }}\right)$ as from Table 5.2 are retrieved

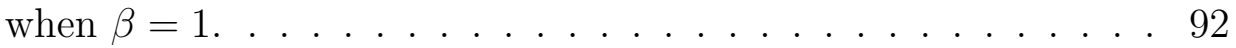

5.5 Table of steady-state temperature and reflection coefficients upon variation of $\mathrm{BN}$ thermal conductivity by factor $\gamma$. The original values for $T_{\text {steady }}$ and $r\left(T_{\text {steady }}\right)$ as from Table 5.2 are retrieved when $\gamma=1$. Note the omission of $r\left(T_{\text {room }}\right)$; this is due to the room temperature reflection not depending on the thermal conductivity of BN. . . . . . . . . . . . . . . 92

5.6 Table of model results of Figure 5.18 upon changing stub depths (noted in order of first, second and third stub turns), optimised to low temperature heating, and a maximised steady-state temperature, compared to the informed model from Figure 5.7 . . . 105

A.1 Table of vector and scalar fields. . . . . . . . . . . . . 135

A.2 Table of fundamental constants. . . . . . . . . . . . . . 136

A.3 Table of material property parameters. . . . . . . . . . . 136

A.4 Table of other frequently used parameters. . . . . . . . . . . 136 


\section{Appendix A}

\section{Nomenclature}

The following tables are a summary of commonly used vector and scalar fields, fundamental constants and other functions.

\begin{tabular}{||c|c|c||}
\hline Symbol & Name & Units \\
\hline \hline$\vec{E}$ & Electric field & $\mathrm{V} / \mathrm{m}$ \\
\hline$\vec{D}$ & Displacement field & $\mathrm{C} / \mathrm{m}^{2}$ \\
\hline$\vec{P}$ & Polarisation field & $\mathrm{C} / \mathrm{m}^{2}$ \\
\hline$\vec{B}$ & Magnetic field & $\mathrm{T} / \mathrm{m}$ \\
\hline$\vec{H}$ & Auxiliary magnetic field & $\mathrm{A} / \mathrm{m}$ \\
\hline$\vec{M}$ & Magnetisation field & $\mathrm{A} / \mathrm{m}$ \\
\hline$\vec{S}$ & Poynting vector & $\mathrm{W} / \mathrm{m}^{2}$ \\
\hline $\overrightarrow{J_{f}}$ & Free current density & $\mathrm{A} / \mathrm{m}^{2}$ \\
\hline$\rho_{f}$ & Free charge density & $\mathrm{C} / \mathrm{m}^{3}$ \\
\hline$V$ & Scalar potential & $\mathrm{V}$ \\
\hline$Q_{E M}$ & Electromagnetic field power & $\mathrm{W}$ \\
\hline$U_{E M}$ & Electromagnetic energy density & $\mathrm{J} / \mathrm{m}^{3}$ \\
\hline$U$ & Thermal energy density & $\mathrm{J} / \mathrm{m}^{3}$ \\
\hline$\vec{q}$ & Heat flux vector & $\mathrm{W} / \mathrm{m}^{2}$ \\
\hline$T$ & Temperature & $\mathrm{K}$ \\
\hline
\end{tabular}

Table A.1: Table of vector and scalar fields. 


\begin{tabular}{||c|c|c|c||}
\hline Symbol & Name & Value & Units \\
\hline \hline$\varepsilon_{0}$ & Permittivity of free space & $8.854 \times 10^{-12}$ & $\mathrm{~F} / \mathrm{m}$ \\
\hline$\mu_{0}$ & Permeability of free space & $1.257 \times 10^{-6}$ & $\mathrm{H} / \mathrm{m}$ \\
\hline$c$ & Speed of light & $2.998 \times 10^{8}$ & $\mathrm{~m} / \mathrm{s}$ \\
\hline$k_{B}$ & Boltzmann constant & $1.381 \times 10^{-23}$ & $\mathrm{~J} / \mathrm{K}$ \\
\hline$\sigma_{B}$ & Stefan-Boltzmann constant & $5.670 \times 10^{-8}$ & $\mathrm{~W} / \mathrm{m}^{2} \mathrm{~K}^{4}$ \\
\hline$h$ & Planck's constant & $6.626 \times 10^{8}$ & $\mathrm{Js}$ \\
\hline$q$ & Electron charge & $1.602 \times 10^{-19}$ & $\mathrm{C}$ \\
\hline
\end{tabular}

Table A.2: Table of fundamental constants.

\begin{tabular}{||c|c|c||}
\hline Symbol & Name & Units \\
\hline \hline$\varepsilon_{r}^{\prime}$ & Real permittivity & - \\
\hline$\varepsilon_{r}^{\prime \prime}$ & Imaginary permittivity & - \\
\hline$\mu_{r}^{\prime}$ & Real permeability & - \\
\hline$\mu_{r}^{\prime \prime}$ & Imaginary permeability & - \\
\hline$\sigma$ & Electrical conductivity & $\mathrm{S} / \mathrm{m}$ \\
\hline$k_{t}$ & Thermal conductivity & $\mathrm{W} / \mathrm{mK}$ \\
\hline$\rho$ & Density & $\mathrm{kg} / \mathrm{m}^{3}$ \\
\hline$c_{p}$ & Heat capacity & $\mathrm{J} / \mathrm{kgK}$ \\
\hline$T_{C}$ & Curie temperature & $\mathrm{K}$ \\
\hline$E_{g}$ & Band gap & $\mathrm{eV}$ \\
\hline
\end{tabular}

Table A.3: Table of material property parameters.

\begin{tabular}{||c|c|c||}
\hline Symbol & Name & Units \\
\hline \hline$f$ & Frequency & $\mathrm{Hz}$ \\
\hline$\omega$ & Angular frequency & $\mathrm{rad} / \mathrm{s}$ \\
\hline$\lambda$ & Wavelength & $\mathrm{m}$ \\
\hline$\vec{k}$ & Wave vector & $1 / \mathrm{m}$ \\
\hline$k$ & Wavenumber & $1 / \mathrm{m}$ \\
\hline
\end{tabular}

Table A.4: Table of other frequently used parameters. 


\section{Appendix B}

\section{Supplementary derivations}

This appendix details various calculations which supplement the mathematical formulae presented in the main text.

\section{B.1 The wave equation}

This section shows the reader how to derive plane wave solutions to the electric and magnetic fields in the Maxwell-Heaviside equations. Consider the following calculation done in free space; so $\rho_{f}=0, \overrightarrow{J_{f}}=\overrightarrow{0}, \varepsilon=\varepsilon_{0}$ and $\mu=\mu_{0}$. Applying the curl operation to (Eq. 2.1.1c) results the following line of calculations

$$
\begin{aligned}
\nabla \times(\nabla \times \vec{E}) & =-\partial_{t} \nabla \times \vec{B} \\
\nabla(\nabla \cdot \vec{E})-\nabla^{2} \vec{E} & =-\partial_{t}^{2}\left(\mu_{0} \vec{D}\right) \\
\nabla^{2} \vec{E} & =\mu_{0} \varepsilon_{0} \partial_{t}^{2} \vec{E}
\end{aligned}
$$

This result is a partial differential equation, known as the wave equation. This equation can be solved by d'Alembert's solution [101] describing wave propagating on a string. The electric field oscillation is entirely mathematically analogous, and the speed of propagation of such an electric field is $c=1 / \sqrt{\varepsilon_{0} \mu_{0}}$. The same calculation can be made for the auxiliary magnetic field;

$$
\nabla^{2} \vec{H}=\mu_{0} \varepsilon_{0} \partial_{t}^{2} \vec{H}
$$

These wave equations are coupled via the latter two Maxwell-Heaviside equations (Eqs. 2.1.1c, 2.1.1d). They are exactly in phase, perpendicular to one 
another and are transverse with respect to the direction of propagation [36].

\section{B.2 Rectangular waveguide wave equation}

This section finds the longitudinal electric and magnetic field differential equations (Eqs. 2.2.2a, 2.2.2b) of a rectangular waveguide through use of relevant vector identities. The Maxwell-Heaviside equations (Eqs. 2.1.1a, 2.1.1b, 2.1.1c, 2.1.1d) are written in terms of the electric and auxiliary magnetic fields in free space.

$$
\begin{aligned}
\nabla \cdot \vec{E} & =0 \\
\nabla \cdot \vec{H} & =0 \\
\nabla \times \vec{E} & =-\mu_{0} \partial_{t} \vec{H} \\
\nabla \times \vec{H} & =\varepsilon_{0} \partial_{t} \vec{E}
\end{aligned}
$$

The auxiliary magnetic field $\vec{H}$ is used instead of the magnetic field $\vec{B}$ to maximise the symmetry of the above equations. To find the longitudinal electric and magnetic field differential equations (analogously to the previous section), start with supposing that there is a harmonic electromagnetic plane wave, propagating in the $z$ direction with wavenumber $k$ and angular frequency $\omega$. The plane waves for such an electromagnetic field can be written as

$$
\begin{aligned}
\vec{E}(x, y, z, t) & =\vec{E}_{0}(x, y) e^{i(k z-\omega t)} \\
\vec{H}(x, y, z, t) & =\vec{H}_{0}(x, y) e^{i(k z-\omega t)}
\end{aligned}
$$

Note that $\partial_{z} \vec{E}=i k \vec{E}$ and $\partial_{t} \vec{E}=-i \omega \vec{E}$. Those identities also apply identically to the auxiliary magnetic field $\vec{H}$. Placing (Eqs. B.2.2a, B.2.2b) along with the aforementioned derivative identities into (Eqs. B.2.1c, B.2.1d) results in two vector equations, which when written out are

$$
\begin{aligned}
\nabla \times\left(\vec{E}_{0}(x, y) e^{i(k z-\omega t)}\right) & =-\mu_{0} \partial_{t}\left(\vec{H}_{0}(x, y) e^{i(k z-\omega t)}\right) \\
\nabla \times\left(\vec{H}_{0}(x, y) e^{i(k z-\omega t)}\right) & =\varepsilon_{0} \partial_{t}\left(\vec{E}_{0}(x, y) e^{i(k z-\omega t)}\right)
\end{aligned}
$$


Then, evaluate each component of the two previous vector equations

$$
\begin{gathered}
\partial_{y} E_{z}-i k E_{y}=i \mu_{0} \omega H_{x} \\
i k E_{x}-\partial_{x} E_{z}=i \mu_{0} \omega H_{y} \\
\partial_{x} E_{y}-\partial_{y} E_{x}=i \mu_{0} \omega H_{z} \\
\partial_{y} H_{z}-i k H_{y}=-i \varepsilon_{0} \omega E_{x} \\
i k H_{x}-\partial_{x} H_{z}=-i \varepsilon_{0} \omega E_{y} \\
\partial_{x} H_{y}-\partial_{y} H_{x}=-i \varepsilon_{0} \omega E_{z}
\end{gathered}
$$

Then solve the set of six equations above for $E_{x}, E_{y}, H_{x}$ and $H_{y}$ in terms of various combinations of derivatives of $E_{z}$, and $H_{z}$.

$$
\begin{aligned}
E_{x} & =\frac{i}{\omega^{2} / c^{2}-k^{2}}\left[k \partial_{x} E_{z}+\omega \mu_{0} \partial_{y} H_{z}\right] \\
E_{y} & =\frac{i}{\omega^{2} / c^{2}-k^{2}}\left[k \partial_{y} E_{z}-\omega \mu_{0} \partial_{x} H_{z}\right] \\
H_{x} & =\frac{i}{\omega^{2} / c^{2}-k^{2}}\left[k \partial_{x} H_{z}-\omega \varepsilon_{0} \partial_{y} E_{z}\right] \\
H_{y} & =\frac{i}{\omega^{2} / c^{2}-k^{2}}\left[k \partial_{y} H_{z}+\omega \varepsilon_{0} \partial_{x} E_{z}\right]
\end{aligned}
$$

Since all components of both the electric and magnetic fields are all written in terms of the longitudinal components, the Gauss' laws for the electric and magnetic field (Eqs. B.2.1a, B.2.1b) can be written out as

$$
\begin{array}{r}
\partial_{x} E_{x}+\partial_{y} E_{y}+\partial_{z} E_{z}=0 \\
\partial_{x} H_{x}+\partial_{y} H_{y}+\partial_{z} H_{z}=0
\end{array}
$$

which when evaluated, using the explicit forms of each field (Eqs. B.2.6a, B.2.6b, B.2.6c, B.2.6d), results in second order partial differential equations for $E_{z}$ and $H_{z}$.

$$
\begin{aligned}
& {\left[\partial_{x}^{2}+\partial_{y}^{2}+\left(\frac{\omega}{c}\right)^{2}-k^{2}\right] E_{z}=0} \\
& {\left[\partial_{x}^{2}+\partial_{y}^{2}+\left(\frac{\omega}{c}\right)^{2}-k^{2}\right] H_{z}=0}
\end{aligned}
$$

These are the partial differential equations which govern the nature of the longitudinal fields (Eqs. 2.2.2a, 2.2.2b) in Chapter 2.2.1. 


\section{B.3 Rectangular waveguide cutoff frequencies}

This section continues from (Eqs. B.2.9a, B.2.9b) to find the cutoff frequencies of electromagnetic radiation in a rectangular waveguide. Start with a TE mode $\left(E_{z}=0\right)$, and use separation of variables on the longitudinal magnetic field of (Eq. B.2.9b).

$$
H_{z}=X(x) Y(y)
$$

which can be substituted into (Eq. B.2.9b) to find

$$
\begin{array}{r}
{\left[\partial_{x}^{2}+\partial_{y}^{2}+\left(\frac{\omega}{c}\right)^{2}-k^{2}\right] X(x) Y(y)=0} \\
\frac{1}{X(x)} \partial_{x}^{2} X(x)+\frac{1}{Y(y)} \partial_{y}^{2} Y(y)+\left(\frac{\omega}{c}\right)^{2}-k^{2}=0
\end{array}
$$

Then, a boundary condition for the electric and magnetic fields, determining the functions $X(x)$ and $Y(y)$ must be found. To do this, consider the fields at the boundary $x=a ; \vec{E}(x=a, y, z)=\left(E_{x}, 0,0\right)$ and $\vec{H}(x=a, y, z)=\left(0, H_{y}, X Y\right)$. Then, write the vacuum form of Ampere's law (Eq. 2.1.1d) component-bycomponent,

$$
\begin{aligned}
\partial_{y} X Y-\partial_{z} H_{y} & =\varepsilon_{0} \partial_{t} E_{x} \\
\partial_{z} 0-\partial_{x} X Y & =\varepsilon_{0} \partial_{t} 0 \\
\partial_{x} H_{y}-\partial_{y} 0 & =\varepsilon_{0} \partial_{t} 0
\end{aligned}
$$

where (Eq. B.3.3b) is of most importance; the condition $Y \partial_{x} X=0$ demands $X(x)$ to have no gradient at $x=a$. The same analysis can be done with the $x=0, y=0$ and $y=b$ cases and this results in fields $X(x)$ and $Y(y)$ to being constant at their respective boundaries. The functions which fit these boundary conditions are

$$
\begin{aligned}
& X(x)=\cos \left(k_{x} x\right) \\
& Y(y)=\cos \left(k_{y} y\right)
\end{aligned}
$$

where the factors $k_{x}$ and $k_{y}$ in (Eqs. B.3.4, B.3.5) must adhere to the boundary conditions set out in (Eq. 2.2.1a, 2.2.1b). This simultaneously 
enforces $k_{x}=m \pi / a$ and $k_{y}=n \pi / b$ with $m, n \geq 0$. These definitions for the respective wavenumbers can be substituted into (Eq. B.3.2) to find

$$
\begin{aligned}
\frac{1}{X(x)} \partial_{x}^{2} X(x)+\frac{1}{Y(y)} \partial_{y}^{2} Y(y)+\left(\frac{\omega}{c}\right)^{2}-k^{2} & =0 \\
-\left(\frac{m \pi}{a}\right)^{2}-\left(\frac{n \pi}{b}\right)^{2}+\left(\frac{\omega}{c}\right)^{2}-k^{2} & =0 \\
\left(\frac{\omega}{c}\right)^{2}-\left(\frac{m \pi}{a}\right)^{2}-\left(\frac{n \pi}{b}\right)^{2} & =k^{2}
\end{aligned}
$$

where the wavenumber $k$ determines the propagation dynamics of an electromagnetic wave. In (Eq. B.3.6) the wavenumber $k$ can be set to zero for a specific angular frequency $\omega \equiv \omega_{m n}$ for each combination of $m$ and $n$.

$$
\omega_{m n}=c \pi \sqrt{\left(\frac{m}{a}\right)^{2}+\left(\frac{n}{b}\right)^{2}}
$$

which can then be expressed as (Eq. 2.2.3) in the main text. Input frequencies which are low $f<f_{m n}$ result in an entirely imaginary wavenumber $k \equiv i k_{i}$, so the fields are rewritten from (Eqs. B.2.2a, B.2.2b) as

$$
\begin{aligned}
& \vec{E}(x, y, z, t)=\vec{E}_{0}(x, y) e^{-k_{i} z} e^{-i \omega t} \\
& \vec{H}(x, y, z, t)=\vec{H}_{0}(x, y) e^{-k_{i} z} e^{-i \omega t}
\end{aligned}
$$

Another important aspect is how energy is carried by an electromagnetic wave. Using the definition of the Poynting vector, and a few calculation steps [36], the time-averaged Poynting vector can be found to be written as

$$
\langle\vec{S}\rangle=\frac{1}{2} \Re\left\{\vec{E} \times \vec{H}^{*}\right\}
$$

with $\vec{H}^{*}$ the complex conjugate of $\vec{H}$, (i.e. $i \rightarrow-i$ in (Eqs. B.3.7a, B.3.7b)). Then, placing the electric and magnetic field expressions of an attenuated wave $\left(f<f_{m n}\right)$ into (Eq. B.3.8) results in

$$
\langle\vec{S}\rangle=\frac{1}{2} \vec{S}_{0}(x, y) e^{-2 k_{i} z}
$$

with $\vec{S}_{0}(x, y)=\Re\left\{\vec{E}_{0}(x, y) \times \vec{H}_{0}^{*}(x, y)\right\}$. This justifies the statement that no electromagnetic field energy is propagated ${ }^{1}$ along the waveguide, as long

\footnotetext{
${ }^{1}$ Altough there may be an exponentially decaying electromagnetic field energy density along the waveguide.
} 
as the frequency of the wave is below the cutoff frequency $f<f_{m n}$ for that specified mode.

\section{B.4 Cylindrical waveguide wave equation}

This section finds the longitudinal electric and magnetic field differential equations (Eqs. 2.2.4a, 2.2.4b) of a cylindrical waveguide through use of relevant vector identities. This derivation is nearly identical to the rectangular case, but more care must be taken due to the added complexity of the vector calculus curl derivative $(\nabla \times)$ in cylindrical coordinates. To find the longitudinal electric and magnetic field differential equations in a cylindrical waveguide, start with a harmonic electromagnetic plane wave, propagating in the $z$ direction with angular frequency $\omega$. The plane waves for the electric and magnetic fields can be written as

$$
\begin{aligned}
& \vec{E}(r, \varphi, z, t)=\vec{E}_{0}(r) e^{i l \varphi} e^{i(k z-\omega t)} \\
& \vec{H}(r, \varphi, z, t)=\vec{H}_{0}(r) e^{i l \varphi} e^{i(k z-\omega t)}
\end{aligned}
$$

Note that $\partial_{z} \vec{E} \equiv i k \vec{E}, \partial_{\varphi} \vec{E} \equiv i l \vec{E}$ and $\partial_{t} \vec{E} \equiv-i \omega \vec{E}$ with $l \geq 0$ the rotational number of the propagating wave. Same as before, these expressions also apply to the $\vec{H}$ field. Placing (Eqs. B.4.1a, B.4.1b) along with the derivative identities into (Eqs. B.2.1c, B.2.1d) results in two vector equations which when written out are

$$
\begin{aligned}
& \nabla \times\left(\vec{E}_{0}(r) e^{i l \varphi} e^{i(k z-\omega t)}\right)=-\mu_{0} \partial_{t}\left(\vec{H}_{0}(r) e^{i l \varphi} e^{i(k z-\omega t)}\right) \\
& \nabla \times\left(\vec{H}_{0}(r) e^{i l \varphi} e^{i(k z-\omega t)}\right)=\varepsilon_{0} \partial_{t}\left(\vec{E}_{0}(r) e^{i l \varphi} e^{i(k z-\omega t)}\right)
\end{aligned}
$$


Then, evaluate each component of the two vector equations

$$
\begin{aligned}
\frac{1}{r} i l E_{z}-i k E_{\varphi} & =i \omega \mu_{0} H_{r} \\
i k E_{r}-\partial_{r} E_{z} & =i \omega \mu_{0} H_{\varphi} \\
\frac{1}{r} \partial_{r}\left(r E_{\varphi}\right)-\frac{i l}{r} E_{r} & =i \omega \mu_{0} H_{z} \\
\frac{1}{r} i l H_{z}-i k H_{\varphi} & =-i \omega \varepsilon_{0} E_{r} \\
i k H_{r}-\partial_{r} H_{z} & =-i \omega \varepsilon_{0} E_{\varphi} \\
\frac{1}{r} \partial_{r}\left(r H_{\varphi}\right)-\frac{i l}{r} H_{r} & =-i \omega \varepsilon_{0} E_{z}
\end{aligned}
$$

by using the definitions of each derivative from above. Then, solve that set of six equations for $E_{r}, E_{\varphi}, H_{r}$, and $H_{\varphi}$ in terms of $E_{z}$ and $H_{z}$.

$$
\begin{aligned}
& E_{r}=\frac{i}{\frac{\omega^{2}}{c^{2}}-k^{2}}\left[k \partial_{r} E_{z}+\frac{i \mu_{0} \omega l}{r} H_{z}\right] \\
& H_{r}=\frac{i}{\frac{\omega^{2}}{c^{2}}-k^{2}}\left[k \partial_{r} H_{z}-\frac{i \varepsilon_{0} \omega l}{r} E_{z}\right] \\
& E_{\varphi}=\frac{i}{\frac{\omega^{2}}{c^{2}}-k^{2}}\left[\frac{i k l}{r} E_{z}-\mu_{0} \omega \partial_{r} H_{z}\right] \\
& H_{\varphi}=\frac{i}{\frac{\omega^{2}}{c^{2}}-k^{2}}\left[\frac{i k l}{r} H_{z}+\varepsilon_{0} \omega \partial_{r} E_{z}\right]
\end{aligned}
$$

Since the fields are written in terms of the longitudinal components, again write out the Gauss' laws for the electric and magnetic field (Eqs. B.2.1a, B.2.1b) in terms of each component in cylindrical coordinates.

$$
\begin{aligned}
& \frac{1}{r} \partial_{r}\left(r E_{r}\right)+\frac{1}{r} \partial_{\varphi} E_{\varphi}+\partial_{z} E_{z}=0 \\
& \frac{1}{r} \partial_{r}\left(r H_{r}\right)+\frac{1}{r} \partial_{\varphi} H_{\varphi}+\partial_{z} H_{z}=0
\end{aligned}
$$

Using the explicit forms of each field (Eqs. B.4.4a, B.4.4b, B.4.4c, B.4.4d), second order differential equations for the longitudinal electric and magnetic 
fields are calculated as

$$
\begin{aligned}
& {\left[r^{2} \partial_{r}^{2}+r \partial_{r}+r^{2}\left(\frac{\omega^{2}}{c^{2}}-k^{2}\right)-l^{2}\right] E_{z}=0} \\
& {\left[r^{2} \partial_{r}^{2}+r \partial_{r}+r^{2}\left(\frac{\omega^{2}}{c^{2}}-k^{2}\right)-l^{2}\right] H_{z}=0}
\end{aligned}
$$

These are the partial differential equations which govern the nature of the longitudinal fields (Eqs. 2.2.4a, 2.2.4b) from Chapter 2.2.2. The solutions to these functions are Bessel functions, and they are discussed in detail in the next section.

\section{B.5 Cylindrical resonance and cutoff frequencies}

This section continues from (Eqs. B.4.5a, B.4.5a) to find the cutoff frequencies of electromagnetic waves of a cylindrical cavity. This time, continue with a TM mode $\left(H_{z}=0\right)$ and let the solution to (Eq. B.4.5a) be

$$
E_{z}(r)=J_{l}(\Gamma r)
$$

where the $\Gamma$ is the radial wavenumber. The function $J_{l}(\chi)$ is known as a Bessel function with unitless argument $\chi$, defined as the solution to the differential equation (Eq. B.4.5a). The conductive boundary conditions from before $\left(E_{\|}=0, H_{\perp}=0\right)$ demand that the longitudinal electric field must be zero at the conductive boundary at radius $R$. This fixes the radial wavenumber $\Gamma \equiv \Gamma_{l m}$ which when multiplied with the radius $R$ results in a zero of the $J_{l}$ 'th Bessel function.

$$
0=J_{l}\left(\Gamma_{l m} R\right)
$$

The zeros of the $l$ 'th order Bessel function where $\chi_{l m}$ is the $m$ 'th zero of the l'th order Bessel function are written as $\Gamma_{l m} R=\chi_{l m}$. The approach to coupling the frequency with the wavevectors is done differently, this time with the general dispersion relation

$$
\frac{\omega^{2}}{c^{2}}=\vec{k} \cdot \vec{k}
$$


with the wavevector written as $\vec{k}=(\Gamma, k)$, with $k$ the propagation wavenumber. Then, since $\Gamma \equiv \Gamma_{l m}=\chi_{l m} / R$ the dispersion relation can be written as

$$
\frac{\omega^{2}}{c^{2}}=\left(\frac{\chi_{l m}}{R}\right)^{2}+k^{2}
$$

Similarly to before, the propagation wavenumber $k$ is zero when the frequency of a specific combination of the $\mathrm{TM}_{\mathrm{lm}}$ mode is given by

$$
f_{l m, \mathrm{TM}}=\frac{c \chi_{l m}}{2 \pi R}
$$

This is the cutoff frequency for an electromagnetic wave propagating within a cylindrical cavity with the $\mathrm{TM}_{\mathrm{lm}}$ mode. The $\mathrm{TE}_{\mathrm{lm}}$ mode is analogously found, but instead with the magnetic field being continuous along the boundary at $r=R$.

$$
f_{l m, \mathrm{TE}}=\frac{c \chi_{l m}^{\prime}}{2 \pi R}
$$

Some of the lowest values of $\chi_{l m}$ and $\chi_{l m}^{\prime}$ are detailed in Table 2.2 for $l=0,1,2$. TM and TE modes with frequencies below the associated cutoff frequencies do not propagate in the cylindrical system. In the context of a microwave furnace resonant cavity, the resonances for a closed-ended cavity are tremendously important for resonant cavity design purposes, as is exemplified in this work. This places a restriction on the wavenumber such that $k \equiv k_{z}=n \pi / L$. Then, the resonant frequencies are determined by placing the new definition of $k$ into (Eq. B.5.4). There are two sets of resonant frequencies, one for the class of TM modes and the other for the class of TE modes.

$$
\begin{aligned}
f_{l m n, \mathrm{TM}} & =\frac{c}{2} \sqrt{\left(\frac{\chi_{l m}}{\pi R}\right)^{2}+\left(\frac{n}{L}\right)^{2}} \\
f_{l m n, \mathrm{TE}} & =\frac{c}{2} \sqrt{\left(\frac{\chi_{l m}^{\prime}}{\pi R}\right)^{2}+\left(\frac{n}{L}\right)^{2}}
\end{aligned}
$$

with $m \geq 1$, and $l, n \geq 0$. These resonant frequencies are the exact expressions as presented in the main text as (Eqs. 2.2.5a, 2.2.5b). The Bessel functions are plotted to specifically note their zeros, as written in Table 2.2. 

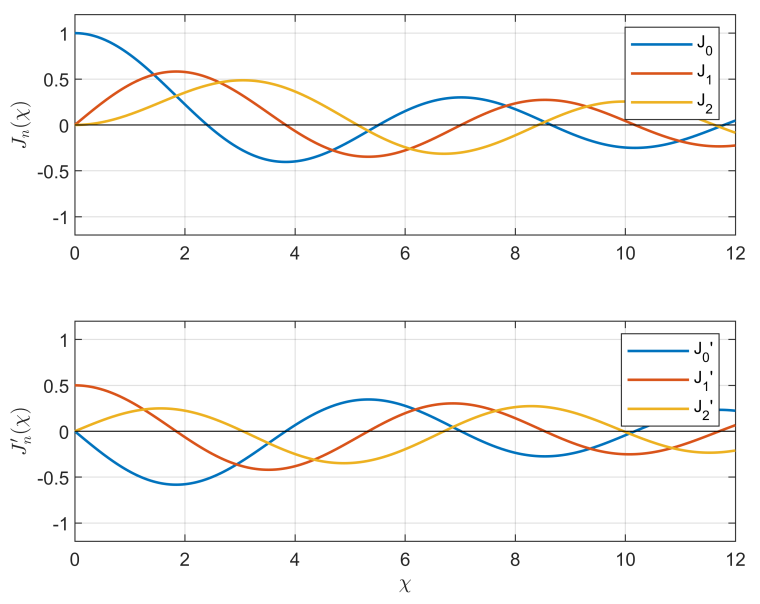

Figure B.1: Plot of the Bessel functions (and the derivatives thereof) of the zeroth, first and second kind. Here the location of the first few zeros as specified in Table 2.2 are apparent.

\section{B.6 Cylindrical beyond-cutoff waveguide}

The functionality of $\mathrm{BC}$ waveguides acts identically to the screening mesh used in commercial microwave ovens [26] to prevent emission of microwave power outside of the enclosed cavity. To understand the attenuation of microwave power as a function of distance along a $\mathrm{BC}$ waveguide, consider the intensity of the fundamental propagating $\mathrm{TE}_{11}$ mode. If a tube is beyond cutoff, this is the least attenuated mode. The $\mathrm{TE}_{11}$ electric field profile along the beyond cutoff waveguide can be written as

$$
\vec{E}_{\mathrm{TE}_{11}}(r, \varphi, z)=\vec{E}_{0} J_{1}\left(r \Gamma_{11}\right) e^{i \varphi} e^{i k z}
$$

The relationship between the wavenumber $k$ and the angular frequency of the wave $\omega_{0}$ within a cylindrical waveguide for the $\mathrm{TE}_{11}$ mode is written as

$$
\frac{\omega_{0}^{2}}{c^{2}}=k^{2}+\Gamma_{11}^{2}
$$

where by setting $k=0$, the cutoff frequency $\left(\omega_{11}=c \Gamma_{11}\right)$ of the waveguide of mode $\mathrm{TE}_{11}$ is found. The wavenumber $k$ can be written in terms of the input frequency $\omega_{0}$ and cutoff frequency $\omega_{11}$.

$$
k=\frac{1}{c} \sqrt{\omega_{0}^{2}-\omega_{11}^{2}}
$$


If frequency $\omega_{0}$ is greater or equal to the cutoff frequency $\omega_{11}$, then the wavenumber $k$ is entirely real and positive. This results in propagation of microwave energy, analogous to the WR340 rectangular wavegude for $2.45 \mathrm{GHz}$ radiation. However, if frequency $\omega_{0}$ is lower than cutoff frequency $\omega_{11}$, the wavenumber will become purely imaginary; (i.e. $k \rightarrow i k_{i}$ ). The expression of the imaginary wavenumber is placed into (Eq. B.6.1) with (Eq. B.6.2) and results in

$$
\vec{E}_{\mathrm{TE}_{11}}(r, \varphi, z)=\vec{E}_{0} J_{1}\left(r \Gamma_{11}\right) e^{i \varphi} e^{-\frac{z}{c} \sqrt{\omega_{11}^{2}-\omega_{0}^{2}}}
$$

Since the electromagnetic energy density of the field $U_{E M}$ is proportional to the square of the electric field (Eq. 2.1.5), and the attenuation amount is proportional to the depth along the $\mathrm{BC}$ waveguide, the attenuation profile of the intensity is thereby written as

$$
U_{E M}(z)=U_{E M, 0} e^{-\frac{2 z}{c} \sqrt{\omega_{11}^{2}-\omega_{0}^{2}}}
$$

with $U_{E M, 0}$ the energy density of the field at the beginning of the $\mathrm{BC}$ waveguide. The energy density of $U_{E M}(z)$ decays exponentially in the beyond cutoff waveguide, proportional to $\exp \left(-2 k_{i} z\right)$. The energy density along the length of the $\mathrm{BC}$ waveguide can be written via in terms of a decibel attenuation with respect to the intensity at the beginning of the waveguide as

$$
\mathrm{dB}\left(z, \omega_{11}\right)=10 \log _{10}\left(\frac{U_{E M}(z)}{U_{E M, 0}}\right)
$$

Then, the combination of (Eqs. B.6.4, B.6.5) results in

$$
\frac{\mathrm{dB}}{z}=-\frac{10}{\ln (10)} \frac{2}{c} \sqrt{\omega_{11}^{2}-\omega_{0}^{2}}
$$

This expression is useful to find by how much the field intensity decays for specific waveguide parameters. The expression (Eq. B.6.6) can also be solved for the length of waveguide $L$ needed to decay the field by some fixed amount of decibels.

$$
L(\mathrm{~dB}, R)=\frac{-c}{2} \frac{\ln (10)}{10} \frac{\mathrm{dB}}{\sqrt{\omega_{11}^{2}-\omega_{0}^{2}}}
$$

The above function can be visualised by fixing the input frequency $f_{0}=$ $2.45 \mathrm{GHz}$ and tracing aspect ratios of constant attenuation. This is best 
represented by plotting the radius $R$ against the length $L$ for each attenuation amount. This is shown in Figure B.2.

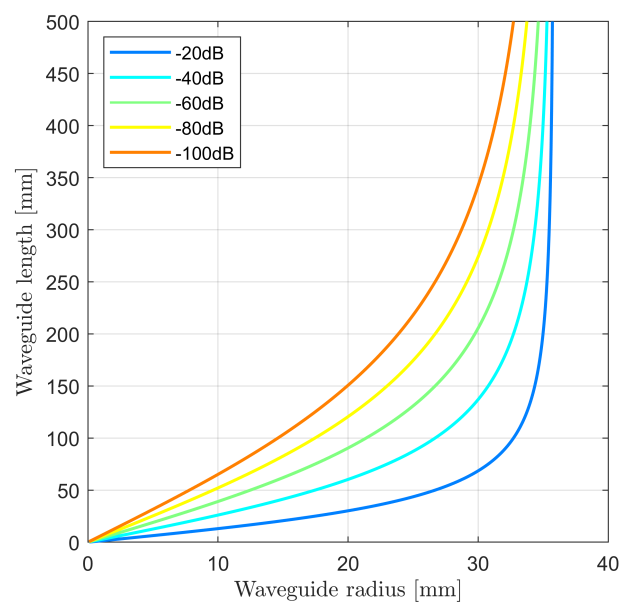

Figure B.2: Combinations of BC waveguide aspect ratios, at fixed attenuation amounts (coloured curves). The maximum radius is given by the cutoff frequency equation $R\left(=c \chi_{l m}^{\prime} / 2 f_{0}\right)=35.86 \mathrm{~mm}$ from (Eq. 2.2.6).

This figure informs mechanical design of $\mathrm{BC}$ waveguide tubes, and shows that the smaller the $\mathrm{BC}$ waveguide is, the shorter it can be to attenuate the intensity of the electromagnetic field by the same amount, when compared to a wider cylindrical waveguide. 


\section{Appendix C}

\section{Mathematical identities}

This appendix provides the basic equations of vector calculus, along with its fundamental theorems. Vector calculus and the associated analysis is used extensively in the field of classical (non-relativistic) electrodynamics. A range of identities is also included, and some were used in calculations in Appendix B.

\section{C.1 Vector calculus: Cartesian Coordinates}

Vector calculus identities in Cartesian coordinates for general scalar field $F(x, y, z)$, and vector fields $\vec{G}(x, y, z)$ and $\vec{H}(x, y, z)$.

$$
\begin{gathered}
\vec{G} \cdot \vec{H}=G_{x} H_{x}+G_{y} H_{y}+G_{z} H_{z} \\
\vec{G} \times \vec{H}=\left[G_{y} H_{z}-G_{z} H_{y}\right] \hat{x}+\left[G_{z} H_{x}-G_{x} H_{z}\right] \hat{y}+\left[G_{x} H_{y}-G_{y} H_{x}\right] \hat{z} \\
\nabla F=\partial_{x} F \hat{x}+\partial_{y} F \hat{y}+\partial_{z} F \hat{z} \\
\nabla \cdot \vec{G}=\partial_{x} G_{x}+\partial_{y} G_{y}+\partial_{z} G_{z} \\
\nabla \times \vec{G}=\left[\partial_{y} G_{z}-\partial_{z} G_{y}\right] \hat{x}+\left[\partial_{z} G_{x}-\partial_{x} G_{z}\right] \hat{y}+\left[\partial_{x} G_{y}-\partial_{y} G_{x}\right] \hat{z}
\end{gathered}
$$




$$
\begin{gathered}
\nabla^{2} F=\partial_{x}^{2} F+\partial_{y}^{2} F+\partial_{z}^{2} F \\
\nabla^{2} \vec{G}=\partial_{x}^{2} G_{x} \hat{x}+\partial_{y}^{2} G_{y} \hat{y}+\partial_{z}^{2} G_{z} \hat{z}
\end{gathered}
$$

\section{C.2 Vector calculus: Cylindrical Coordinates}

Vector calculus identities in cylindrical coordinates for general scalar field $F(r, \varphi, z)$ and vector fields $\vec{G}(r, \varphi, z)$ and $\vec{H}(r, \varphi, z)$.

$$
\begin{aligned}
& \vec{G} \cdot \vec{H}=G_{r} H_{r}+G_{\varphi} H_{\varphi}+G_{z} H_{z} \\
& \vec{G} \times \vec{H}=\left[G_{\varphi} H_{z}-G_{z} H_{\varphi}\right] \hat{r}+\left[G_{z} H_{r}-H_{r} G_{z}\right] \hat{\varphi}+\left[G_{r} H_{\varphi}-H_{r} G_{\varphi}\right] \hat{z} \\
& \nabla F=\partial_{r} F \hat{r}+\frac{1}{r} \partial_{\varphi} F \hat{\varphi}+\partial_{z} F \hat{z} \\
& \nabla \cdot \vec{G}=\frac{1}{r} \partial_{r}\left(r G_{r}\right)+\frac{1}{r} \partial_{\varphi} G_{\varphi}+\partial_{z} G_{z} \\
& \nabla \times \vec{G}=\left[\frac{1}{r} \partial_{\varphi}\left(r G_{z}\right)-\partial_{z} G_{\varphi}\right] \hat{r}+\left[\partial_{z} G_{r}-\partial_{r} G_{z}\right] \hat{\varphi}+\frac{1}{r}\left[\partial_{r}\left(r G_{\varphi}\right)-\partial_{\varphi} G_{r}\right] \hat{z} \\
& \frac{1}{r} \partial_{r}\left(r \partial_{r} F\right)+\frac{1}{r^{2}} \partial_{\varphi}^{2}+\partial_{z}^{2} F \\
& \nabla^{2} \vec{G}=\left(\nabla^{2} G_{r}-\frac{G_{r}}{r^{2}}-\frac{2}{r^{2}} \partial_{\varphi} G_{\varphi}\right) \hat{r}+\left(\nabla^{2} G_{\varphi}-\frac{G_{\varphi}}{r^{2}}-\frac{2}{r^{2}} \partial_{\varphi} G_{r}\right) \hat{\varphi}+\nabla^{2} G_{z} \hat{z}
\end{aligned}
$$

\section{C.3 Fundamental theorems of vector calculus}

Assume existence of scalar field $F\left(x_{1}, x_{2}, x_{3}\right)$, and vector fields $\vec{G}\left(x_{1}, x_{2}, x_{3}\right)$, $\vec{H}\left(x_{1}, x_{2}, x_{3}\right)$, and $\vec{I}\left(x_{1}, x_{2}, x_{3}\right)$ in general coordinates $\left(x_{1}, x_{2}, x_{3}\right)$. Define volume $V$ with surface area $\Sigma$, surface area element $d S$, unit vector $\hat{r}$ along contour $\partial \Sigma$ and normal vector $\hat{n}$ of said surface $\Sigma$. Define path $l$ from point 
$\vec{a}=\left(x_{1}^{(1)}, x_{2}^{(1)}, x_{3}^{(1)}\right)$ to point $\vec{b}=\left(x_{1}^{(2)}, x_{2}^{(2)}, x_{3}^{(2)}\right)$ with unit vector $\hat{u}$ along that path.

Curl theorem.

$$
\oint_{\partial \Sigma}(\vec{G} \cdot \hat{r}) d r=\iint_{\Sigma}((\nabla \times \vec{G}) \cdot \hat{n}) d S
$$

Divergence theorem

$$
\iiint_{V}(\nabla \cdot \vec{G}) d V=\iint_{\Sigma}(\vec{G} \cdot \hat{n}) d S
$$

Gradient theorem

$$
\int_{\vec{a}}^{\vec{b}}((\nabla F) \cdot \hat{u}) d l=F(\vec{b})-F(\vec{a})
$$

\section{C.4 Vector identities}

Assuming existence of scalar field $F\left(x_{1}, x_{2}, x_{3}\right)$ and vector fields $\vec{G}\left(x_{1}, x_{2}, x_{3}\right)$, $\vec{H}\left(x_{1}, x_{2}, x_{3}\right)$, and $\vec{I}\left(x_{1}, x_{2}, x_{3}\right)$ in general coordinates $\left(x_{1}, x_{2}, x_{3}\right)$.

$$
\begin{gathered}
\nabla^{2} \vec{G}=(\nabla \cdot \nabla) \vec{G} \\
\nabla \times(\nabla \times \vec{G})=\nabla(\nabla \cdot \vec{G})-\nabla^{2} \vec{G} \\
\nabla \cdot(\nabla \times \vec{G})=0 \\
\nabla \times(\nabla F)=0 \\
\nabla \cdot(\vec{G} \times \vec{H})=\vec{G} \cdot(\nabla \times \vec{H})-\vec{H} \cdot(\nabla \times \vec{G}) \\
\vec{G} \cdot(\vec{H} \times \vec{I})=\vec{H} \cdot(\vec{I} \times \vec{G})=\vec{I} \cdot(\vec{G} \times \vec{H}) \\
\vec{G} \times(\vec{H} \times \vec{I})=\vec{H}(\vec{G} \cdot \vec{I})-\vec{I}(\vec{G} \cdot \vec{H})
\end{gathered}
$$




\section{Appendix D}

\section{Relevant software interfaces}

This appendix shows the interfaces of the three most significant softwares used in this work; LabVIEW, COMSOL Multiphysics and MATLAB, then discusses the primary application of each.

\section{D.1 National Instruments LabVIEW}

The version of LabVIEW used in this work is NI LabVIEW 2017 (64-bit). A license to use the software was provided by Robinson Research Institute.

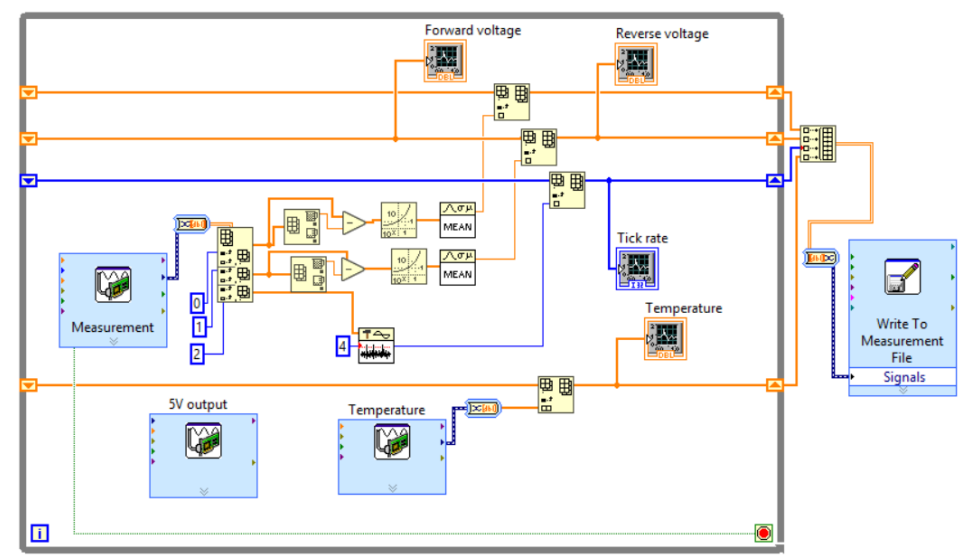

Figure D.1: Block diagram used to acquire data during research.

The software was used to acquire the data from the AD8317 RF-DC logarithmic detectors, K-type thermocouple measurement and flow meter is LabVIEW. The LabVIEW software used in this work essentially used the following logic; acquire AD8317 and flow meter signals at $10 \mathrm{kHz}$ via NI USB-6001 and the 
thermocouple measurement via NI USB-TC01 at $1 \mathrm{~Hz}$. Time average the AD8317 voltage signals over 1 second, and sum the total ticks produced by the flow meter to find the water volume flow rate. Then, plot the transient voltage measurements and thermocouple readings over time. After stopping the program, the data was automatically logged into an empty excel spreadsheet.

\section{D.2 COMSOL Multiphysics}

The version of COMSOL used in this work was originally COMSOL Multiphysics 5.5, but was upgraded to COMSOL Multiphysics 5.6 towards the end of the

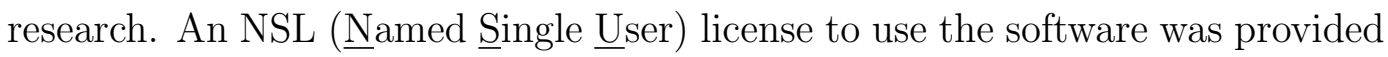
by Dr. Chris Bumby.

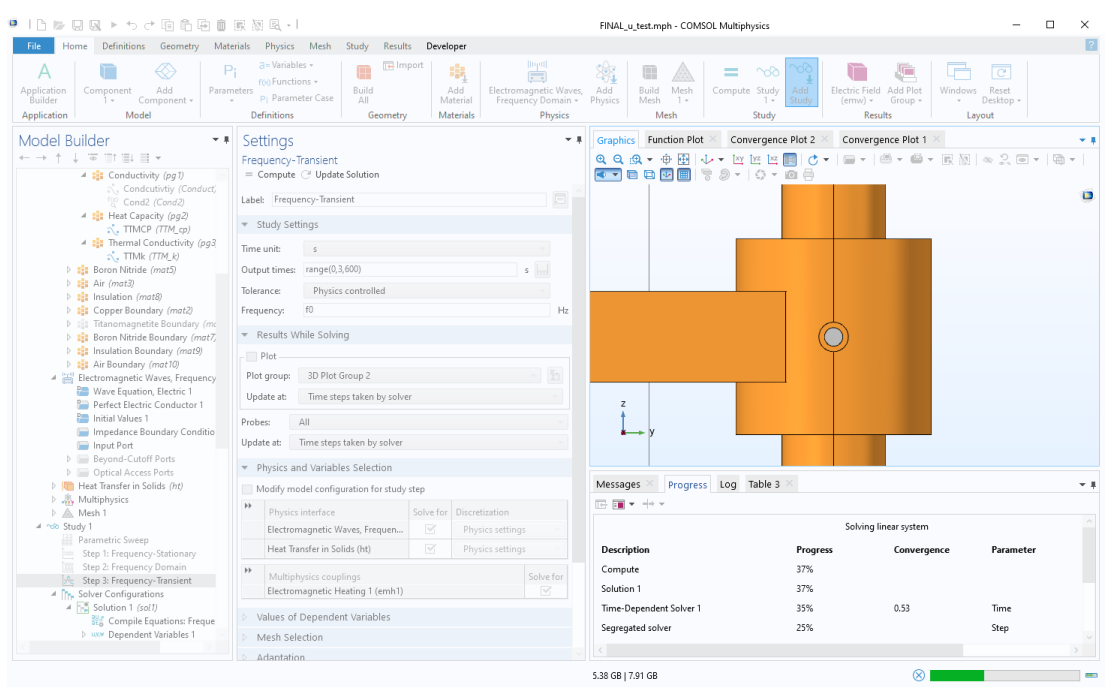

Figure D.2: Standard user interface for COMSOL Multiphysics 5.6 while software is simulating a transient model.

The general process in building a model is to firstly define the physics to be solved for, and to choose the general solver method. After this, the geometry is built, and the relevant materials are added into the domains and boundaries. Then, a mesh grid is built for those domains and boundaries. This is done either automatically by the software, or by a user-defined meshing process. Temperature dependent material properties can be incorporated via a 'look-up-table', in which COMSOL references a .csv or .txt file for the material property for a specific temperature. Then, the software is executed, and a simulation is produced. For simple electromagnetic problems this simulation 
can take under 10 seconds, but complex transient systems may take up to 2 hours to complete.

The most common causes for errors in simulation (either a terminating simulation, or an unphysical model) are due to misplaced/unphysical boundary conditions, significantly varying material properties over a short temperature range (i.e. $\mu_{r}(T)$ around $T_{C}$ ), or compounding errors throughout transient multiphysics simulations. Care must therefore be taken to model the physics in COMSOL accurately, however if done correctly results in a powerful tool for modelling highly complex systems such as the microwave heating of TTM ironsand done in this thesis.

\section{D.3 MathWorks MATLAB}

The version of MATLAB used in this work was MATLAB R2018a. The license to use this software was provided by Victoria University of Wellington. The purpose of MATLAB in this work was to post-process and visualise the data presented in this thesis from computational and experimental results. As such, most of the figures produced in this work were directly produced in MATLAB.

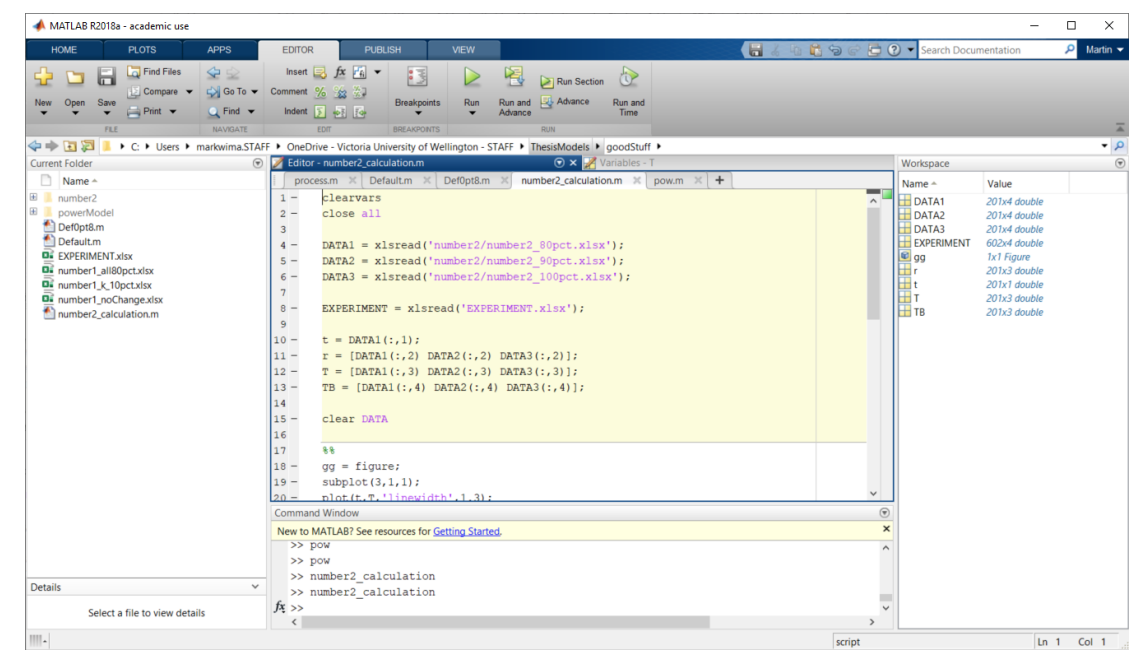

Figure D.3: Standard user interface for MATLAB R2018a.

The general process of each program was to clear the workbench, close all open MATLAB figures, then (if necessary) load the relevant dataset. In some cases, some post-processing of data was done. After this, the data is plotted. 
